University of Louisville

ThinkIR: The University of Louisville's Institutional Repository

Electronic Theses and Dissertations

8-2011

\title{
Mechanistic insights into copper-induced regression of heart hypertrophy.
}

\author{
Katherine Bourcy \\ University of Louisville
}

Follow this and additional works at: https://ir.library.louisville.edu/etd

\section{Recommended Citation}

Bourcy, Katherine, "Mechanistic insights into copper-induced regression of heart hypertrophy." (2011). Electronic Theses and Dissertations. Paper 133.

https://doi.org/10.18297/etd/133

This Doctoral Dissertation is brought to you for free and open access by ThinkIR: The University of Louisville's Institutional Repository. It has been accepted for inclusion in Electronic Theses and Dissertations by an authorized administrator of ThinkIR: The University of Louisville's Institutional Repository. This title appears here courtesy of the author, who has retained all other copyrights. For more information, please contact thinkir@louisville.edu. 


\title{
MECHANISTIC INSIGHTS INTO COPPER-INDUCED REGRESSION OF HEART HYPERTROPHY
}

\author{
By \\ Katherine Bourcy \\ B.S., University of Louisville, 2003 \\ M.S., University of Louisville, 2007

\begin{abstract}
A Dissertation
Submitted to the Faculty of the

School of Medicine of the University of Louisville

In Partial Fulfillment of the Requirements
\end{abstract} \\ For the Degree of \\ Doctor of Philosophy \\ Department of Pharmacology \& Toxicology \\ University of Louisville \\ Louisville, KY
}

August 2011 


\title{
MECHANISTIC INSIGHTS INTO COPPER-INDUCED REGRESSION OF HEART HYPERTROPHY
}

\author{
By \\ Katherine Bourcy \\ B.S., University of Louisville, 2002 \\ M.S., University of Louisville, 2008
}

A Dissertation Approved on

July 26,2011

By the following Dissertation Committee:

\begin{tabular}{c}
\hline Y. James Kang, D.V.M., Ph.D. \\
\hline John W. Eaton_M.D., Ph.D. \\
\hline David W. Hein, Ph.P. \\
\hline Zhao-Hui (IDe) Song,Ph.D. \\
\hline
\end{tabular}

Dale A. Schuschke, Ph.D. 


\section{ACKNOWLEDGMENTS}

I want to thank my dissertation director, Dr. Kang for all his help, guidance and encouragement. I don't know what the future holds, but I have learned so much during my time here and I know that the future is bright. I would also like to extend my gratitude to all my committee members, Drs. Eaton, Hein, Song and Schuschke, for their helpful comments, guidance, their service on my committee, as well as their patience with the last minute scheduling of committee meetings and schedule coordinating messes.

I would also like to thank Dr. Cai for his assistance in helping analyze many, many samples using LC/MS-MS, and Dr. Feng for countless hours of assistance and advice, especially while I was trying to get my research up and running and struggling to optimize experimental conditions. I am also very much indebted to Yang Zhou for all her help, especially when I first joined the lab. Additionally, Li Zhan and Lala Hussain have also played an important role in this chapter of my life. And while he was not on my committee, I would also like to thank Dr. Michal Hetman for the life-changing conversation.

I would like to thank my friends for their patience and forbearance with my erratic schedule, for their laughter and encouragement that kept me going when all I could think of was how much I wanted to quit. It's been a long time coming, but the tables have finally turned and the day has arrived. I would especially like to thank my sister, Joi. We have been through so much together, from the depths of the pit to the blazing road to the 
top that we're on now; I have no doubt I wouldn't have made it without your cheer, wry sense of humor and contagious laughter.

I told everyone I would jokingly say, "This Ph.D. is brought to you by Sugar-Free Red Bull, sponsored in part by Starbucks Coffee, Heine Brothers, Highland Coffee, Sunergos and Five-Hour Energy!" in my dissertation acknowledgements, but I was actually half serious. In addition to the love and support of all my friends, I would not have survived graduate school without coffee and/or caffeine.

Everyone else who has made this journey even the slightest bit more bearable, who was always ready with your quick wit or an encouraging word...you know who you are, and I love you dearly... 


\title{
ABSTRACT \\ MECHANISTIC INSIGHTS INTO COPPER-INDUCED REGRESSION OF HEART HYPERTROPHY
}

\author{
Katherine Bourcy
}

July 26,2011

Previous studies have shown that copper $(\mathrm{Cu})$ supplementation at physiologically relevant levels reverses cardiac myocyte hypertrophy induced by phenylephrine (PE), and that this effect was VEGF-dependent. Yet, the amount of VEGF in the media was unchanged. However, we observed that $\mathrm{Cu}$ caused an increase in the ratio of VEGFR1:VEGFR-2 as well as an increase in PKG-1 activity. PKG-1 activity is associated with the regression of cardiac myocyte hypertrophy. The present study was undertaken to test the hypothesis that VEGFR-1 is associated with PKG-1 and their association is involved in $\mathrm{Cu}$ induced regression of cardiomyocyte hypertrophy. Human cardiac myocytes $(\mathrm{HCM})$ in cultures were exposed to phenylephrine (PE) at a final concentration of 100 $\mu \mathrm{M}$ for 48 hours to induce cell hypertrophy. Copper sulfate at a final concentration of 5 $\mu \mathrm{M}$ was added to the hypertrophic HCM cultures for 24 hours with the concomitant presence of PE to reverse the hypertrophy. Both hypertrophic and hypertrophic-reversed HCM cells underwent immunoprecipitation using anti-VEGFR-1 antibody or anti-PKG-1 antibody. The immune complex underwent gel-electrophoresis separation and Western blotting and LC-MS/MS analysis. Proteomic analysis identified Vimentin in the immune 
complexes that immunoprecipitated with VEGFR-1 and PKG-1. This study thus demonstrates that the association between VEGFR-1 with PKG-1 is mediated by Vimentin, and that Vimentin plays a critical role in copper regression of cardiac myocyte hypertrophy. 


\section{TABLE OF CONTENTS}

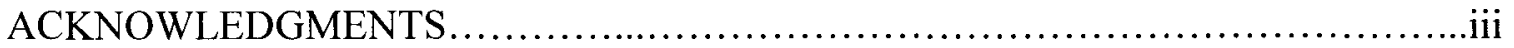

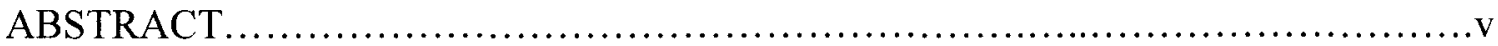

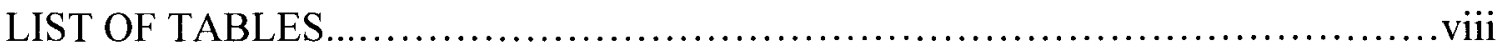

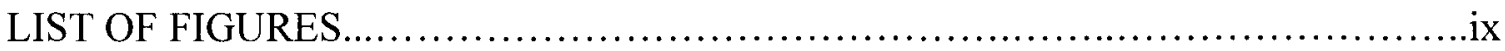

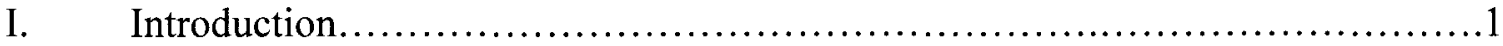

1.1 The Heart $\&$ Heart Failure.......................................................

1.2 Element \#29: Cuprum ........................................................... 10

1.3 Vascular Endothelial Grown Factor and its Receptors...........................16

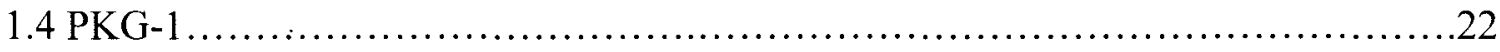

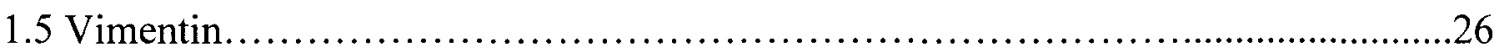

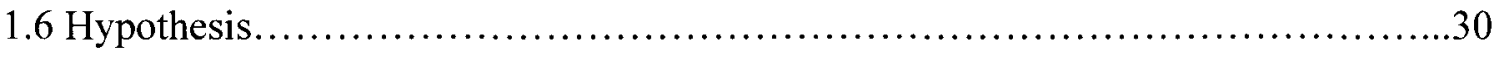

II. Materials and Methods.......................................................

III. Results....................................................................49

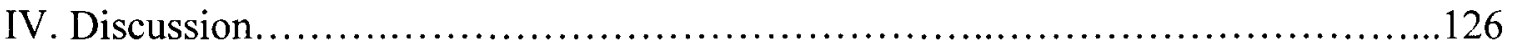

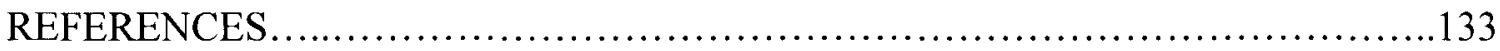

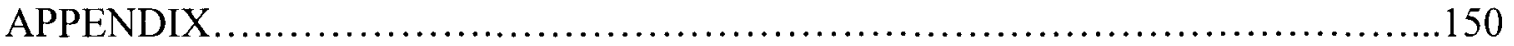

CURRICULUM VITAE..................................................... 153 


\section{LIST OF TABLES}

\section{Page}

Table 1: PKG-1 pull down................................................ 85

Table 2: PKG-1 pull down....................................................

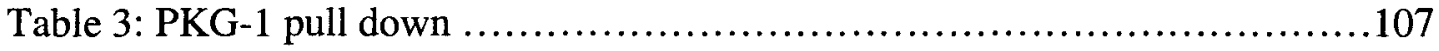

Table 4: VEGFR-1 pull down.............................................112 


\section{LIST OF FIGURES}

FIGURE

Page

1. Quantitative analysis of VEGF secretion.................................50

2. Schematic representation of VEGFR signaling............................51

3. Fluorescent microscopic images of VEGFR-1 and VEGFR-2 $\ldots \ldots \ldots \ldots \ldots \ldots \ldots . \ldots 3$

4. Flow cytometric analysis of VEGFR-1 and VEGFR-2 .......................54

5. Flow cytometric analysis of VEGFR-1 and VEGFR-2 $\ldots \ldots \ldots \ldots \ldots \ldots \ldots \ldots \ldots$

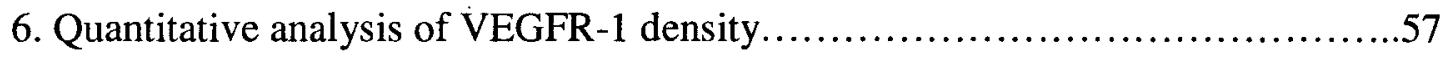

7. Quantitative analysis of VEGFR-2 density ...............................58

8. Regression of PE-induced cell hypertrophyby siRNA targeting VEGFR-2 .......59

9. Regression of PE-induced cell hypertrophy by siRNA targeting VEGFR-2 .......60

10. Regression of PE-induced cell hypertrophy by siRNA targeting VEGFR-2 ......61

11. Requirement of VEGF...............................................63

12. Requirement of VEGF...............................................64

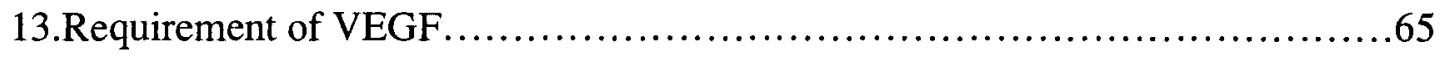

14. Requirement of VEGF.............................................66

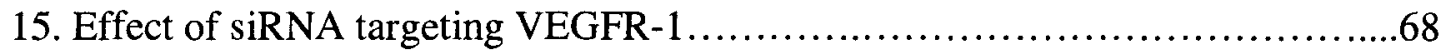

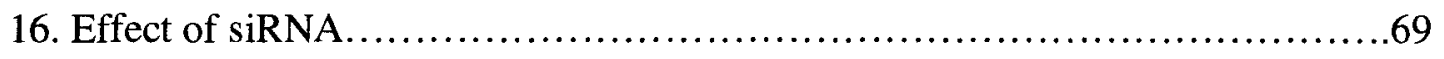

17. Quantitative analysis of VEGFR-1 post-siRNA targeting VEGFR-1............70

18. Quantitative analsys of cell size post-siRNA targeting VEGFR-1..............71 
19. Quantitative analysis of cell size post-siRNA targeting VEGFR-1 $\ldots \ldots \ldots \ldots \ldots . \ldots 72$

20. Quantitative analysis of cell size post-siRNA targeting VEGFR-1 ..............73

21. Inhibitory effect of siRNA targeting VEGFR-1 on PKG-1 activity...............75

22. Antagonistic effect of PKG-1 inhibitor on regression of cell hypertrophy ........76

23. Antagonistic effect of PKG-1 inhibitor on regression of cell hypertrophy........77

24. Antagonistic effect of PKG-1 inhibitor on regression of cell hypertrophy .........78

25. Antagonistic effect of PKG-1 inhibitor on regession of cell hypertrophy.........79

26. Antagonistic effect of PKG-1 inhibitor on regression of cell hypertrophy........80

27. Antagonistic effect of PKG-1 inhibitor on regression of cell hypertrophy........81

28. Complete schematic representation of VEGFR signaling in cardiac myocyte.....82

29. Vimentin Western blot.............................................. 123

30. Co-immunoprecipitation of Vimentin and PKG-1...................... 124

31. Co-immunoprecipitation of Vimentin and VEGFR-1 ..................... 125 


\section{INTRODUCTION}

Heart disease is the leading cause of death in the United States, killing more than 927,000 people every year. While it does not receive as much attention as cancer, cardiovascular disease claims more lives than all cancers combined, fewer than 560,000 deaths ${ }^{1}$. With these statistics come with a tremendous cost to society in the form of health care costs, as health care spending is projected to reach $\$ 4.7$ trillion dollars by the year $2020^{2}$. Additionally, this also comes with personal costs as well, as this disease is so prevalent it is highly unlikely anyone will go through life and somehow not be affected by heart disease.

The heart is the first organ to develop during embryogenesis ${ }^{3}$. Throughout the lifetime of the organism, there will be many changes in stress and demand on the heart, and in some circumstances the heart will undergo growth, or hypertrophy. There are two kinds of hypertrophy: physiological and pathological ${ }^{4}$. Physiological hypertrophy is both normal and reversible ${ }^{5}$. In response to an increased workload and stress, the heart muscle (myocardium) will become thicker to preserve normal heart function. This can occur in athletes, for example, who train intensively. However, once the stimulus is removed, the heart will eventually go back to its normal size. In contrast, pathological hypertrophy is neither normal nor it is considered reversible. In the case of pathological hypertrophy, the stimulus is often some type of injury to the heart, such as a myocardial infarction. The heart undergoes an adaptive response and becomes enlarged in an attempt to 
preserve function. However, pathological hypertrophy is an important milestone in the progression to heart failure, and once developed cannot be reversed ${ }^{6}$. Moreover, the prognosis for those with heart failure is often worse than those with cancer ${ }^{7}$. While there are current therapies for heart failure, such as beta-blockers, angiotensin converting enzyme (ACE) inhibitors, diuretics and digoxin, these can, in some cases, buy the patient some time but will not stop or reverse the progression to heart failure. Other options such as heart transplantation are often prohibitive in nature for no other reason than the cost. The past two decades have brought better understanding and improved approaches to treating cardiac hypertrophy and heart failure. As it turns out, while it was previously thought that pathological hypertrophy was irreversible, there are data suggesting that it may, in fact, be reversible.

Copper is a critical micronutrient and is essential for life ${ }^{8}$. Roughly twenty enzymes within the human body contain copper, and of those, ten require copper to function. Additionally, because these enzymes are found within most tissues, the effects of copper deficiency are systemic and widespread ${ }^{9}$. Copper is essential for the development of healthy bones ${ }^{10}$, skin $^{11}$, and blood vessels ${ }^{12}$. The cardiovascular system is especially sensitive to copper levels ${ }^{13}$. The strongest evidence for this can be found by the extreme consequences of copper deficiency mainly manifesting as damage to the heart and blood vessels ${ }^{14}$. It is interesting to note that while the recent health benefits of red wine are often touted as being due to resveratrol, red wine is made with the skins of the grape intact; copper-based fungicides are often used on wine grapes thereby making red wine a good source of copper. For centuries, copper has been used to treat a wider 
variety of maladies. As it turns out, it may also be useful in healing an injured, and maybe even a failing heart.

Previous studies have shown that copper $(\mathrm{Cu})$ supplementation at physiologically relevant levels can reverse cardiac myocyte hypertrophy induced by phenylephrine (PE), and that this phenomenon was VEGF-dependent. This observation recaptures the in vivo observation that $\mathrm{Cu}$ supplementation can reverse cardiac hypertrophy induced by pressure overload in a mouse model, and this effect is also VEGF-dependent ${ }^{15}$. However, there are fundamental differences between in vivo observations and those observed in cardiac myocytes grown in cultures. Coronary angiogenesis clearly plays a critical role in the regression of cardiac hypertrophy, however there are no blood vessels in cell cultures, which indicates VEGF has a direct effect on cardiac myocytes in the regression of cell hypertrophy.

Because the amount of VEGF in the media was unchanged, we looked to VEGF receptors. We observed that $\mathrm{Cu}$ caused an increase in the ratio of VEGFR-1:VEGFR-2 as well as an increase in PKG-1 activity, which is associated with the regression of cardiac myocyte hypertrophy. The present study was undertaken to test the hypothesis that VEGFR-1 is associated with PKG-1 and their association is involved in $\mathrm{Cu}$ induced regression of cardiomyocyte hypertrophy.

When the phenomenon of the regression of cardiac hypertrophy was first observed, it was observed in vivo in a mouse model, then in rat cardiac myocytes. We used human cardiac myocytes. Because there are differences between human and rat cells, we first established a phenotype. Indeed, $\mathrm{Cu}$ supplementation also reversed cardiac myocyte hypertrophy induced by PE in human cardiac myocytes. 
Human cardiac myocytes (HCM) in cultures were exposed to phenylephrine (PE) at a final concentration of $100 \mu \mathrm{M}$ for 48 hours to induce cell hypertrophy. Copper sulfate at a final concentration of $5 \mu \mathrm{M}$ was added to the hypertrophic HCM cultures for 24 hours with the concomitant presence of PE to reverse the hypertrophy. Both hypertrophic and hypertrophic-reversed HCM cells underwent immunoprecipitation using anti-VEGFR-1 antibody or anti-PKG-1 antibody. The immune complex underwent gel-electrophoresis separation and Western blotting and LC-MS/MS analysis.

Initially, we had hypothesized that VEGFR-1 and PKG-1 were directly linked, and this is, in part, how cardiac myocyte hypertrophy is reversed. But we found that the link was not direct, but indirect. Functional proteomic analysis identified Vimentin in the immune complex likely involves VEGFR-1 and PKG-1. This study thus demonstrates that the association between VEGFR-1 with PKG-1 is mediated by Vimentin, and that Vimentin plays a critical role in copper regression of cardiac myocyte hypertrophy.

While initially thought to be nothing more than a 'decoy' receptor, these data suggest that VEGFR-1 plays a critical role in the regulation of cardiac myocyte size. And once thought to be nothing more than an intermediate filament that helped provide scaffolding for the cell, this study suggests Vimentin, a known $\mathrm{Cu}$-binding protein, may play a critical role in signaling pathways in cardiac myocytes, perhaps acting as a molecular switch, shutting down hypertrophic pathways, while allowing regression signaling to prevail and thus reversing the size of hypertrophic cardiac myocytes. 


\section{1-1. THE HEART \& HEART FAILURE}

The heart is a pump that rivals any man-made machine. Before the day is done, the heart will beat over 100,000 times, propelling blood throughout the pulmonary and systemic circulation, supplying the body with life-supporting oxygen and nutrients. During the average lifetime, the human heart will beat roughly 2.5 billion times, without cessation. It is the first organ to develop during embryogenesis, and all subsequent events throughout the life of the organism are dependent upon its function.

In its most simplistic view, the heart can be organized into three layers: the endocardium, the innermost layer of tissue that lines the chambers of the heart; the myocardium, the middle, muscular layer of the heart; and the epicardium, which is composed of the connective tissue surrounding the heart. Most of the heart is composed of non-muscle cells that constitute more than $2 / 3$ of the total populations of the cardiac cells. However, the contractile muscle cells, which are known as myocytes or cardiac myocytes, are less than $1 / 3$ of the total populations of the cardiac cells, but account for nearly $75 \%$ of cardiac volume. The rest of the heart is composed of pacemaker and conducting tissue, which are involved in the initiation and conduction of electrical impulses to the ventricles; blood vessels and extracellular space. 
Cardiac myocytes consist of mainly contractile proteins. $\mathrm{Z}$ lines are found at the ends of the myocyte. From the $\mathrm{Z}$ line, thin actin filaments stretch inward and form cross-links with the much larger and thicker myosin filaments. Myosin filaments, which typically resemble golf clubs lying on their side, extend from the center of the myofibril toward the $\mathrm{Z}$ line but do not touch them. Titan elastic molecules indirectly link myosin fibers to the $\mathrm{Z}$ line. The release of calcium ions initiates muscle contraction. The heads of the myosin fibers flex and move actin fibers such that the $\mathrm{Z}$ lines move towards one another.

The concentration of intracellular calcium ions is regulated in a series of interconnecting events. The sarcolemma, or external cell membrane contains calcium channels. With the arrival of each action potential and wave of electrical excitation, these channels open, allowing a small amount of calcium to enter the cell. Electrical impulses make their way into the cardiac myocyte by way of invaginations called $\mathrm{T}$ tubules, which are in close proximity to the sarcoplasmic reticulum, which stores calcium ions. A conformational change in the ryanodine receptor triggers the release of more calcium from the sarcoplasmic reticulum, a process called calcium-induced calcium release. Contractions are triggered by an increase in internal cytosolic calcium.

Just as the release of calcium initiates contraction, relaxation of contractile proteins occurs when calcium is uptaken into the longitudinal component of the sarcoplasmic reticulum. While the increase in calcium concentrations acts upon the sarcoplasmic reticulum, it also triggers the phosphorylation of the regulatory protein phospholamban, which in turn activates ATP-dependent calcium uptake pumps of the 
sarcoplasmic reticulum. Phorphorylated phospholamban blocks the inhibition of the calcium pump of the sarcoplasmic reticulum. Calcium is removed and relaxation occurs.

The heart is a dynamic organ; its component muscle is capable of growing and adapting to changing workloads. The contraction of the myocardium plays a critical role in regulating the circulatory system by ejecting blood from the left ventricle; the tone of the peripheral arterioles provides most of the resistance that the heart must work against. In order to adapt to changes in workload, or arterial tone, the heart will undergo remodeling. The main players in this remodeling are cardiac myocytes. Enlargement of the heart is attributed to enlargement of individual cardiac myocytes In certain circumstances, such as pregnancy or increased aerobic activity, the heart will undergo physiological hypertrophy. This type of hypertrophy is normal, reversible, and associated with normal or enhanced cardiac functionality. Conversely, in response to disease-induced stimuli, such as pressure overload or myocardial infarction, congenital malformations, myocarditis, or diabetic cardiomyopathy, pathological hypertrophy may result. These stimuli damage the heart. In an attempt to compensate for the damage, the heart increases the pump function along with an irreversible enlargement of the size, or undergoes pathological hypertrophy. Pathological hypertrophy ultimately leads to cardiac dilation and loss of contractility. It is associated with an upregulation of fetal genes, cardiac dysfunction, decreased contractile function and increased mortality. It is an early milestone in the clinical progression to heart failure and in many cases, sudden cardiac death ${ }^{16}$. Once 
a patient develops pathological hypertrophy, it cannot be reversed and the progression to heart failure becomes inevitable $e^{17}$.

At the most basic understanding, heart failure is when the heart can no longer deliver the necessary nutrients and oxygen to peripheral tissues in the body. In the Western world, adult cardiac disease, most frequently in the form of heart failure, kills more people than all cancers combined ${ }^{18}$. Patients with heart failure fall under two major categories: systolic heart failure or diastolic heart failure, and the distinction is whether the dominant functional problems result from decreased contractility (systolic failure) or decreased relaxation (diastolic failure). Nearly a third of patients with evidential heart failure have preserved ejection fractions, and their symptoms are either largely or entirely stem from diastolic dysfunction ${ }^{19}$. This negative lusitropic state is attributed to structural changes in the heart such as fibrosis, hypertrophy and infiltrative processes. Additionally, subcellular aberrations in repolarization-relaxation coupling are often present in many cases ${ }^{20,21}$.

Heart failure can involve the right, left or both ventricles of the heart, most often both ventricles. The right ventricle of the heart pumps blood to the lungs where blood is reoxygenated. The left ventricle of the heart pumps oxygen-rich blood and nutrients to the rest of the body. Right ventricle heart failure often follows long-term high blood pressure in the pulmonary arteries, or pulmonary hypertension, causing a strain on the right side of the heart. In the event of right ventricle heart failure, the heart cannot pump enough blood to the lungs. Left ventricle heart failure can be caused by coronary artery disease as well as many other factors, and similarly with 
left ventricle heart failure, the heart cannot pump enough blood to the peripheral tissue.

Heart failure is the most expensive cardiovascular disorder in the United States with annual expenditures estimated to be in excess of $\$ 29.6$ billion $^{22,23}$. It is a leading cause of death in the U.S. ${ }^{24}$ and is quickly becoming more prevalent globally ${ }^{25}$. By the year 2020, it is projected that deaths from cardiovascular disease will exceed those from infectious and parasitic diseases for the first time throughout the world with the exception of sub-Saharan Africa ${ }^{26,27}$. While there are current treatments available for heart failure ranging from pharmacological intervention to heart transplantation, they all carry inherent risks and hefty price tags. Medications used to treat heart failure may exacerbate the condition. Additionally, medications used to treat heart maladies other than heart failure, such as antiarrhythmics and calcium channel blockers, may induce heart failure ${ }^{28}$. Currently, no treatment is available that can reverse hypertrophy, thereby halting the progression to heart failure. 


\section{1-2. ELEMENT \#29: CUPRUM}

Cuprum or what is more commonly known as copper is a transition metal. It is used as an electrical conductor, as well as in the construction of metal alloys such as bronze and brass. While Hippocrates was known for prescribing copper compounds to treat disease ${ }^{29}$ as well as to prevent pregnancy ${ }^{30}$ the use of copper compounds such as copper carbonate, copper oxide, copper sulfate and copper chloride predates the ancient Egyptians. In fact, the use of copper as a medicinal agent can be traced as far back as Aztec civilization where it was used to treat sore throats. Throughout history, copper compounds have been used to treat a wide variety of diseases; Ancient Egyptians used copper for the sterilization of drinking water as well as the treatment of wounds; the Romans recorded numerous medicinal uses for copper; in Persia and India it was used to treat maladies from boils to venereal ulcers ${ }^{31}$.

After iron and zinc, copper is the third most common trace element in the human body. Copper is an important micronutrient for man and it is essential for life. The most compelling evidence for this can be found by the severe consequences of copper deficiency mainly manifesting as damage to the heart and blood vessels. At least twenty enzymes within the human body contain copper, and of those ten are dependent upon copper to function. Additionally, because these enzymes are found within most tissue, the effects of copper deficiency are systemic and widespread. Low copper levels can result in numerous pathological states including, but not limited to: poor neuronal myelination, reduced aortic elasticity, impaired immune 
responses, decreased motor function, blood vessel abnormalities and cardiac hypertrophy $\mathrm{h}^{32,33,34}$.

The cardiovascular system is especially vulnerable to alterations in copper status. The essentiality of copper in the cardiovascular system has been demonstrated in numerous clinical and experimental studies ${ }^{35,36,37,38,39,40,41,42,43,44}$. Because copper facilitates the absorption and utilization of iron, it follows that copper deficiency is also associated with anemia, decreasing the amount of oxygen that is delivered to tissue $^{45,46}$. Furthermore, copper deficiency decreases clotting capabilities after injury, increasing the amount of blood lost ${ }^{47}$. And once clots are formed, their ability to dissolve is also impaired. Copper deficient blood vessels are inherently leaky, and exacerbate swelling.

Yet the strongest evidence for the importance of copper to the cardiovascular system has been observed in laboratory mouse model with pre-established hypertrophic cardiomyopathy induced by ascending aortic constriction. Copper supplementation alone reversed cardiac hypertrophy and this recovery occurred despite sustained pressure overload ${ }^{48}$. This suggests that copper supplementation prevents heart failure by reversing cardiac hypertrophy. Upon further investigation, it was discovered that cytochrome c oxidase recovery is essential to this coppersupplementation-induced recovery, and is also validated by other studies which demonstrated that copper deficiency reduced the hearts ability to utilize energy and contribute to reduced coronary function ${ }^{49,50,51,52}$.

As a transition metal, it also plays an important role as a cofactor in a wide variety of biological reduction and oxidation (redox) reactions. However, some suggest that 
this also contributes to its potential for toxicity. In cases of copper overload, copper may cause oxidative damage to cells in a Fenton-type redox reaction. However, it is interesting to note that there is no free copper within the cell ${ }^{53}$, and it is tightly controlled.

Copper metabolism was of little interest to researchers until the 1920s after the discovery that copper was required for the formation of hemoglobin in rats ${ }^{54}$. Subsequent studies in the 1930s and 40s were done in humans amounted to studies in the ADME (Absorption, Distribution, Metabolism and Excretion) of copper ${ }^{55,56,57,58}$. Much of the debate regarding the metabolic fate of copper in the 1930s and 40s are not much different than the debates that occur today, and the same can be said for minimum dietary copper requirements; amounts vary from $0.63 \mathrm{mg} / \mathrm{d}$ to $2.65 \mathrm{mg} / \mathrm{d}$. The current RDI for copper is $0.9 \mathrm{mg}$ per day ${ }^{59}$. Though, it goes without saying that the study of whole body copper metabolism in humans is inherently difficult. Tissues available for analysis are few, and this alone greatly limits what can be learned. The few tissues which can be sampled, for example skin and the liver, are only available through invasive means and even then, concentrations of copper in tissue can only give limited information. Also, it is impossible to determine which copper was already in the body, and which copper came from a meal without the use of isotopic tracers.

Copper is obtained by the body from the diet ${ }^{60}$ and drinking water from copper piping. It is absorbed through the duodenum and intestinal mucosa and transported to the liver through the portal vein. The liver is the most important organ for copper homeostasis and is primarily responsible for removal of excess copper from the body. 
In the liver, it was thought that copper is incorporated into ceruloplasmin and released into the bloodstream as Cu-ceruloplasmin, and is taken up by various tissues within the body. However studies have demonstrated that ceruloplasmin is not essential for copper transport to extrahepatic tissue as aceruloplasminemia did not exhibit signs of organ system copper deficiency ${ }^{61}$. Copper is also released into the blood from the intestine in the form of $\mathrm{Cu}$-albumin, $\mathrm{Cu}$-histidine, $\mathrm{Cu}$-transcuprein, as well as other unidentified complexes. Most dietary copper is excreted into the gastrointestinal tract and lost in the bile. After combining with the small amount of copper that is excreted from intestinal and pancreatic fluids and intestinal cells, it is eliminated from the body. Copper concentrations are highest in the liver, followed by the brain, kidney and heart. As the amount of copper intake increases, so does absorption, however a higher percentage is absorbed when intake is low. Studies suggest the primary factor to influence copper absorption is the amount of available copper.

Whole-body copper metabolism can vary depending on conditions, such as pregnancy, and disease states. Metabolic defects, such as Wilson disease or Menkes syndrome are two frequently encountered copper malmetabolism disease conditions. Wilson disease is a rare, autosomal recessive disorder affecting normal copper metabolism. Typically, excess copper is excreted in the bile, but with this disease, mutations in the putative copper transporting P-type ATPase, ATP7B cause copper to build up in the liver. Over time, the liver is damaged and copper is released directly into the bloodstream causing damage to the kidneys, brain and eyes. Symptoms can appear as early as 4 years old, but onset can also be as late as 40 years of age. A rusty, brown ring around the cornea of the eye is the most characteristic sign of the 
disease. On the other extreme is Menkes syndrome. This syndrome is caused by a defect in the ATP7A gene, which manifests as a mutation in the MNK protein, a Ptype ATPase. This results in copper deficiency as copper distribution and transport into the cells are inadequate. Consequently, the brain and other parts of the body do not get enough copper. Symptoms include structural abnormalities in the hair (Menekes syndrome is also known as "kinky hair syndrome" as it results in brittle, kinky hair), abnormal bone structure and severe neurological impairment. Most Menkes patients die in early childhood. Both of these disorders illustrate how critical copper metabolism and balance is within the body, and the need for strict homeostatic control of copper.

The exact mechanism by which copper is taken into cells, and its journey within the cell is not completely understood, yet there is still much that is known. Under normal conditions, copper is thought to be transported into intestinal cells through the high affinity copper transporter, CTR1 and bound to cytosolic copper chaperones. COX17 and SCOI/II are believed to act as chaperones, delivering copper to the mitochondria and facilitating the incorporation of copper into cytochrome c oxidase. $\mathrm{Cu}$-ATPases ATP7A and ATP7B receive copper from ATOX1, transporting copper into the lumen of the secretory pathway of the trans Golgi network, as well as removing excess copper from the cell. CCS (copper chaperon for superoxide dismutase), as the name implies, delivers copper to cytosolic $\mathrm{Cu}, \mathrm{Zn}$ superoxide dismutase 1 (SOD1).

Due to the regulation of body copper stores, indices of copper (save under extreme conditions) are resistant to change ${ }^{62}$. Even after dietary intake of copper 
went from 0.8 to $7.5 \mathrm{mg} / \mathrm{d}$, traditional indexes of copper status, such as ceruloplasmin, plasma copper, erythrocyte superoxide dismutase and urinary copper excretion were not significantly different ${ }^{63}$. However, recent studies have demonstrated that there is no reliable biomarker for copper. Neither cuproenzymes nor plasma copper reflect copper status ${ }^{64,65,66}$. While traditionally, ceruloplasmin has been used as a biomarker for copper status, it is subject to season changes ${ }^{67}$, and is also age-dependent ${ }^{68}$. The only known exact indicator of copper status is copper content in the liver, which is not readily measured ${ }^{69}$. Alternatively, a recent promising candidate as a biomarker for copper status is CCS. In a rat feeding study, it was shown to increase in a dose-response manner as copper intake decreased in erythrocytes and liver ${ }^{70}$. Furthermore, there was a concomitant decrease in SOD1 levels, and the authors of the study have suggested using the CCS:SOD1 ratio as an indices of copper deficiency. Thus far, it seems to be the most promising candidate of not only a measure of copper deficiency, but excess as well. 


\section{1-3. VASCULAR ENDOTHELIAL GROWTH FACTOR (VEGF) AND ITS RECEPTORS}

Vascular Endothelial Growth Factor (VEGF) is a robust mitogen for endothelial cells derived from lymphatics, arteries and veins, although it does not elicit a consistent and appreciable mitogenic response in any other types of cells ${ }^{71,72,73,74,75,76,77}$. Because of its ability to induce vascular leakage in guinea pig skin, VEGF is also known as Vascular Permeability Factor (VPF) ${ }^{78,79}$. Additional effects of VEGF include the stimulation of hexose transport in the vascular endothelium ${ }^{80}$, promote the expression of VCAM (Vascular Cell Adhesion Molecule-1), ICAM (Intracellular Adhesion Molecule-1) and Eselectin, all of which are adhesion molecules, via NF-kB with PI3K (phosphotidylinositol-3 kinase)-mediated suppression, in endothelial cells ${ }^{81},{ }^{82}$ and may regulate or promote monocyte chemotaxis ${ }^{83}$. In vitro studies have shown VEGF induces vasodilation in a dose-dependent manner and can produce hypotension, tachycardia and decreased cardiac output when injected intravenously in rats ${ }^{84}$. However in vitro studies have shown VEGF does not affect contractility in isolated rat hearts ${ }^{85}$. It is important to note, though that these hemodynamic effects are not limited to VEGF whereas other angiogenic factors can also induce hypotension and nitric oxide-mediated vasodilation ${ }^{86}$.

There are several VEGF isoforms, each arising as a result of alternative splicing, VEGF121, 145, 165, 183, 189 and 206 . They are characterized by the presence of eight conserved cysteine residues and a cystine knot residue ${ }^{87}$. Native VEGF is a heparinbinding, basic, homodimeric glycoprotein ${ }^{88}$. VEGF165, the other major isoform, shares 
the same properties. VEGF165 appears to be the most abundant and potent isoform in man, acting as an angiogenic cytokine regulating vascular permeability, ${ }^{89}$ followed by VEGF121 and $189^{90}$. VEEGF is induced by hypoxia and cytokines, such as TNF- $\alpha$, IL1, 6 and 8 , and oncostatin $\mathrm{M}^{91}$. Despite numerous isoforms, VEGF can be easily categorized by the biological property of their ability to bind heparin and heparin-sulfate. VEGF121 is the most soluble, weakly acidic and does not bind heparin or the extra cellular matrix $(\mathrm{ECM})^{92,93}$, however, VEGF189 and 206 are more basic, bind heparin and are completely sequestered to the $\mathrm{ECM}^{94}$. VEGF165 does bind heparin, and roughly $50-70 \%$ is bound to the cell surface and the ECM. Both VEGF145 and 165 induce angiogenesis in vivo, however VEGF145 is mainly expressed in cells derived from reproductive organs, as is VEGF206. These findings suggest there are two different mechanisms by which VEGF may become available to endothelial cells: by protease activation following cleavage of the larger isoforms, or freely diffusible proteins (VEGF121, VEGF165). When comparing VEGF165 to VEGF121 or VEGF110, a 50-fold reduction in potency was observed in an endothelial cell growth assay ${ }^{95}$. These results allude to VEGF exerting structural and functional heterogeneity in order to yield a controlled biological response.

The biological consequences of VEGF are mediated by two receptors, VEGFR-1 (also known as Flt-1) and VEGFR-2 (also known as Flk-1/KDR), both of which are receptor tyrosine kinases (RTK) and are how VEGF induces biological responses ${ }^{96}$. VEGFR-3 is a member of RTK family, but it does not bind VEGF instead binding its homologues, VEGFC and VEGFD ${ }^{97}$. In mice lacking VEGFR-2, the development of hematopoietic and endothelial cells is impaired ${ }^{98}$. It was observed in Flt-1 null mice overgrowth of endothelial cells and blood vessel disorganization ${ }^{99}$. While our 
understanding of these receptors is incomplete, the distinct phenotypes of VEGFR-1 and VEGFR-2 knockout mice suggest these receptors have differing biological functions. It then follows that the downstream signaling transduction pathways are also different and have considerably different signaling properties ${ }^{100}$. VEGFR-1 and 2 are similar in structure with an extracellular domain that is comprised of seven immunoglobulin-like domains, one transmembrane region and a consensus tyrosine kinase sequence that is split by a kinase-insert domain ${ }^{101,102}$. Both receptors bind VEGF with a very high affinity. VEGFR-1 has the highest affinity for rhVEGF165, with a Kd of 10-20 pM ${ }^{103}$. VEGFR-2 binds VEGF with a slightly lower affinity, with a Kd of $75-125 \mathrm{pM}^{104}$.

For almost thirty years, it has been known that $\mathrm{Cu}$ stimulates angiogenesis in the avascular cornea of rabbits ${ }^{105}$. This has been demonstrated in subsequent studies in both experimental and clinical settings ${ }^{106}$. Another study showed that $\mathrm{Cu}$ stimulates VEGF synthesis in human keratinocytes ${ }^{107}$. The exact mechanism by which $\mathrm{Cu}$ regulates VEGF expression is unknown. It is known, however, that HIF-1 $\alpha$ is a required transcription factor for VEGF ${ }^{108,109}$, ergo oxygen tension plays a critical role in VEGF regulation. During normoxia conditions HIF-1 $\alpha$ undergoes hydroxylation at a proline residue by prolyl hydroxylase (PHD), which requires oxygen, iron and 2-oxoglutarate to function $^{110}, 111,112,113$. This increases (and is also required for) HIF-1 $\alpha$ binding to von Hippel-Lindau (VHL) tumor suppressor protein, thereby targeting HIF-1 $\alpha$ for proteasomal degradation $^{114}$. However, under hypoxic conditions, PHD cannot function. HIF-1 $\alpha$ is neither hydroxylated nor targeted for degradation; it accumulates in the cells and its activity increases ${ }^{115}$. Studies in the lab have demonstrated that $\mathrm{Cu}$ 
supplementation reverses cardiac hypertrophy in part by $\mathrm{Cu}$ chaperone for superoxide dismutase-1(CCS)-mediated HIF-1 $\alpha$ activation of VEGF ${ }^{116}$.

Our preliminary studies have demonstrated the requirement of VEGF for the reversal of cardiac hypertrophy in primary culture of neonatal rat cardiomyocytes. Because these cells are treated with VEGF in culture without the presence of blood vessels, the effect of VEGF is independent of angiogenesis. Additionally, a recent study showed that chronic activation of PKG-1 prevents and reverses cardiac hypertrophy in hearts exposed to sustained pressure overload ${ }^{117}$. Upon further investigation into the mechanism, it was discovered that this effect was also mediated by the upregulation of VEGF.

While investigating the potential mechanisms by which $\mathrm{Cu}$ reverses cardiac hypertrophy, we observed $\mathrm{Cu}$ deficiency suppressed and $\mathrm{Cu}$ repletion restored VEGF expression in the heart ${ }^{118}$. The heart is designed such that every cell is in contact with a blood vessel, supplying the cardiomyocyte with a constant flow of oxygen and nutrients. Consequently, coronary angiogenesis is critical. And a delicate balance between cardiac growth and angiogenesis must be maintained. During the acute phase of cardiac hypertrophy, angiogenesis is increased. However, if angiogenesis is reduced, the heart undergoes pathological remodeling. Further, if cardiac growth and angiogenesis are not coordinated, the transition to heart failure occurs ${ }^{119}$. This disruption suppresses VEGF. It is also interesting to note that blocking VEGF promotes and VEGF administration delays the transition to heart failure in pressure overloaded mouse models ${ }^{120,121}$. In our preliminary studies, we observed that $\mathrm{Cu}$ supplementation enhanced VEGF expression, reversed hypertrophic cardiomyopathy and restored angiogenesis despite pressure 
overload in mouse models with AAC-induced cardiac hypertrophy and dysfunction.

Together, this suggests that $\mathrm{Cu}$ is involved in the regulation of VEGF expression in the heart.

VEGF is critical in $\mathrm{Cu}$ induced reversal of cardiac hypertrophy. It also plays a role in and is required for the cell growth that leads to cardiac hypertrophy. This suggests that VEGF plays a dual role in controlling the physiological function of cardiomyocytes. Under stress conditions, it is required for cell growth, but also decreases the size of hypertrophied cardiomyocytes. While the importance of VEGF-induced angiogenesis has been demonstrated for the amelioration of ischemic myocardium ${ }^{122,123}$, VEGF alone is not a good strategy for the reversal of cardiac hypertrophy ${ }^{124}$. VEGF, which is also known as vascular permeability factor, can induce vascular leakage ${ }^{125,126}$, and in cases of mitochondrial dysfunction, the addition of VEGF culminates in the creation of leaky vessels $^{127}$. However, an independent and direct effect of VEGF that is independent of angiogenesis on cardiomyocytes should play a crucial role in the retrogression of hypertrophic cardiomyopathy.

The main focus of this dissertation is the identification and characterization of VEGFR mediated signaling pathways involved in the reduction of hypertrophied cardiomyocyte cell size and thus, reversal of cardiac hypertrophy. In order to gain a comprehensive understanding of these signaling pathways, VEGFR associated pathways of normal cardiomyocytes were compared to that of hypertrophied cardiomyocytes. We observed that $\mathrm{Cu}$-induced regression of heart hypertrophy is VEGF-dependent. Yet, the amount of VEGF in cultures did not change. VEGF mediates its effects through its receptors. It was later determined that $\mathrm{Cu}$ causes a change in the ratio of VEGFR- 
1:VEGFR-2 and hypothesized that this leads to a switch in signaling pathways from growth to the regression of cell hypertrophy. Additionally, siRNA targeting VEGFR-2, which elevated the ratio of VEGFR-1:VEGFR-2 differentiated but also reproduced the Cu-induced reversal of cardiac myocyte hypertrophy. Further, Anti-VEGF antibody blocked the reversal of cardiac myocyte hypertrophy induced by VEGFR-2 knock down. Finally, siRNA targeting VEGFR-1 ablated Cu-induced reversal of hypertrophy, further indicating the important role VEGFR-1 plays in the regression of cardiomyocyte hypertrophy. 


\section{1-4. PKG-1}

Before the day is done, the heart will beat over 100,000 times, faithfully fulfilling its role in delivering oxygen and nutrients to peripheral tissues. With this also comes the need to adapt rapidly to ever changing demands and stresses, from the stress induced by physiological or pathological stimuli. Within the heart lies regulators that can aid in this process, cyclic nucleotides $3^{\prime}, 5^{\prime}$-monophosphates such as cAMP and cGMP and their respective effector enzymes, protein kinase A (PKA) and protein kinase G (PKG). The pathway of cAMP/PKA controls excitation-contraction coupling associated with the phosphorylation of voltage-gated calcium channels, phospholamban, ryanodine receptors and sarcomeric proteins, which increase and drives calcium cycling through the sarcoplasmic reticulum and triggers contraction and relaxation. Conversely, the pathway of cGMP/PKG acts as a myocardial decelerator, countering cAMP-dependent-stimulation and signaling different pathways that act to blunt growth, while increasing relaxation ${ }^{128}$.

PKG-1 is believed to be the primary target of cyclic GMP (cGMP). This serine/threonine protein kinase is activated by paracrine and autocrine stimuli, which increase nitric oxide (NO) and cGMP. In mammals, there are three molecular forms of PKG: PKG-1 $\alpha$, PKG-1 $\beta$, and PKG-II. PKG- $1 \alpha$ and PKG- $1 \beta$ result from alternative mRNA splicing and differ only by about 100 amino acids in the Nterminal dimerization domain ${ }^{129,130,131}$; and PKGII arises from a different gene locus. 
PKG-1 is the major isoform in cardiac myocytes and will therefore be the focus of this chapter ${ }^{132,133}$. The aforementioned N-terminal homodimerization domains of PKG- $1 \alpha$ and $\beta$ consequently determine their isoform-specific interactions with cell substrates and docking proteins. While PKG-1 $\alpha$ and PKG- $1 \beta$ have indistinguishable cGMP binding sites, PKG1 $\alpha$ binds cGMP with a 10 -fold higher affinity than PKG$1 \beta^{134,135}$. Additionally, PKG-1 $\alpha$ dimerization is subject to ROS-regulation, its dimerization forming a disulfide bond between reactive $\mathrm{Cys}^{42}$ residues that adjoin in the enzyme homodimer. PKG-1 $\beta$ is neither ROS-sensitive nor does it contain reactive Cys at this position ${ }^{136}$.

In 1986, Murry et al observed the phenomenon that would later become known as cardioprotection ${ }^{137}$. Since then, a growing body of evidence has shown the complex signaling pathways involved in the hypertrophic regulation of the heart, and that it is dependent upon multiple components. One important and well-studied pathway is cyclic GMP and cGMP-dependent protein kinase (PKG). Studies in mice with increased cGMP synthesis, which upregulated natriuretic peptide receptor signaling showed overactivity of cGMP blunted hypertrophy in vitro, and despite abnormal loading conditions in vivo ${ }^{138,139}$. Similar observations are made with PKG-1 activation $^{140}$, and conversely, PKG-1 inhibition exacerbates hypertrophy ${ }^{141}$. A recent study that also demonstrated the important role that PKG plays in the regulation of cardiac hypertrophy showed that hyperstimulation of the PKG-1 pathway can prevent and reverse cardiac hypertrophy ${ }^{98}$. However, it did not abolish hypertrophy induced by overexpression of Akt in vivo. Additionally, further studies into the mechanism of this phenomenon showed that it was VEGF-dependent ${ }^{142,143}$. However, it has been 
suggested that other signaling pathways may play a role in the observed antihypertrophic effects, as one study reported that cardiac hypertrophy was not exacerbated by the deletion of PKG-1 in cardiac myocytes ${ }^{144}$.

It has been reported that PDE5, a cGMP-specific phosphodiesterase type 5 enzyme, is upregulated in congestive heart failure ${ }^{145}$, pulmonary hypertension ${ }^{146}$ and right ventricle hypertrophy ${ }^{147}$. The preconditioning-like cardioprotective effects of sildenafil against injury sustained by ischemia-reperfusion were observed in the intact hearts of animals ${ }^{148}$. It was observed in other studies in adult mice that sildenafil had a direct effect in the heart by protecting against necrosis and apoptosis through activation of the NO-signaling pathway, and this effect was ablated by gene knock down of NO synthase ${ }^{149}$. While sildenafil has demonstrated powerful cardioprotective effects against ischemia-reperfusion injury ${ }^{150,151,152,153}$ post-infarction heart failure ${ }^{154}$ and cardiomyopathy induced by doxorubicin ${ }^{155}$, these observations are not limited to sildenafil as other PDE5 inhibitors, vardenafil ${ }^{156}$ and tadalafil ${ }^{157}$ limit or reduce infarct sizes.

As there are activators of cardiac hypertrophy, there are also negative modulators, and their inactivation may play an important role in cardiac hypertrophy and failure. One important negative modulator is GSK3 $\beta$, a serine/threonine kinase that regulates cardiac development, cell development and cycling, gene transcription, apoptosis and metabolism $^{158}$. Normally, when GSK $3 \beta$ is active, it inhibits hypertrophy. However, when it is phosphorylated by Akt or PKA, its activity is suppressed and the inhibition of hypertrophic pathways is removed, paving the way for hypertrophy to develop. 
Studies have suggested that GSK3 $\beta$ plays an integral role in regulating VEGF, which may be critical for cardioprotection ${ }^{159}$ and also may be another clue to understanding the mechanism by which $\mathrm{Cu}$ can reverse cardiac hypertrophy. Further, it was observed that Akt phosphorylation was insignificantly abrogated by PKG inhibitor KT5823 or shRNA-PKG ${ }^{160}$. VEGFR-1 plays a critical role in modulating VEGF/Akt signaling ${ }^{161}$ by preventing excessive activation of Akt. This is in agreement with other studies that showed acute activation of Akt exerted protective effects against both myocardial infarction and ventricular dysfunction following ischemia-reperfusion in vivo and in vitro ${ }^{162,163}$. Sildenafil resulted in PKGindependent increases in Akt phosphorylation, suggesting that perhaps PKG-1 and VEGFR-1 act synergistically to switch signaling pathways from hypertrophy to regression in cell size. 


\section{1-5. VIMENTIN}

The cytoskeleton is unique to eukaryotic cells. Filling the role of both muscle and skeleton, it aids in both movement and stability of the cell. The cytoskeleton is composed of three types of filaments: microfilaments, microtubules and intermediate filaments ${ }^{164}$. Microfilaments, the smallest of the three, are roughly 3-6 $\mathrm{nm}$ in diameter. The most well-known examples of microfilaments are actin and myosin, which carry out the roles of contraction and cytokinesis in the cell. Microtubules are cylindrical in appearance, which are $20-25 \mathrm{~nm}$ in diameter and are composed of the protein tubulin, whose subunits are termed alpha and beta. Microtubules are thought to act as a scaffolding to determine cell shape, and act as a "track" on which organelles and vesicles within the cell to move. Additionally, during mitosis, microtubules form spindle fibers that separate the chromosomes during anaphase. Finally, intermediate filaments, named for their diameter because they fall between microfilamens and microtubules in size, are roughly $10 \mathrm{~nm}$ in diameter, and are thought to provide the cell with tensile strength ${ }^{165}$.

Intermediate filaments constitute approximately $1 \%$ of the total protein of cells. In humans, there are more than 50 intermediate filaments genes which are expressed in nearly all cells within the body ${ }^{166}$. And while microfilaments and microtubules are highly conserved and similar within cells of a particular species, intermediate

filaments exhibit diversity in their numbers, sequence and abundance ${ }^{167}$. However, 
they do share a common structure: a dimer consisting of two $\alpha$-helical chains in parallel and intertwined, making a coiled-coil rod. Though the structure may be similar, the properties vary and can be quite unique. For example, keratin intermediate filaments found in hair are highly insoluble, but nuclear lamin intermediate filaments are dynamic and dissociate and reform in a cell cycledependent manner.

Vimentin is the most abundant intermediate filament protein. It is widely expressed and once thought to be a static element involved mainly in structural processes. Initially it was believed to play a main role in providing stability for the architecture of the cell, yet some studies report that knockout models in which the animals do not express Vimentin exhibit a normal phenotype, develop and reproduce normally. Therefore it was concluded that Vimentin is not necessary for the viability of eukaryotic cells ${ }^{168}$. However, under certain circumstances, Vimentin knockout mice do exhibit phenotypic abnormalities under specific conditions, such as injury ${ }^{169,170}$. This suggests Vimentin has a specialized biological function that contributes to specific dynamic cellular processes ${ }^{171}$. With respect to the cardiovascular system, Vimentin is an integral part of the vascular endothelium, and has been suggested to play an important role in shear stress-induced responses, such as arterial remodeling ${ }^{172}$.

In endothelial cells, Vimentin is phosphorylated at serine and threonine residues, and its secretion is increased by phosphatase inhibitors, and conversely, blocked by the inhibitor G06983, which is specific for protein kinase C. This is consistent with other findings that phosphorylation of Vimentin affects its intracellular localization; 
Vimentin is phosphorylated by numerous protein kinases and is a substrate for protein kinase $\mathrm{C}(\mathrm{PKC})$. Further studies have observed that PKC directly phosphorylates Vimentin at Ser/Thr residues, possibly marking it for secretory pathways. Also, other studies have demonstrated that IL-10, an anti-inflammatory cytokine mediates its effects by inhibiting the PKC pathway. TNF- $\alpha$ is a well-known cytokine which functions in opposition to IL-10 in macrophages. It was observed that even at extremely low doses, TNF- $\alpha$ induced the secretion of Vimentin by as much as 120fold in human monocytes. Yet current models for TNF- $\alpha$ signaling do not transpire through PKC thus suggesting both PKC and TNF- $\alpha$ activate pathways that mediate the secretion of Vimentin.

Hypoxia is a physiological stimulus that can occur in various conditions, including but not limited to ischemia, anemia, high altitude and lung disease. While these conditions can alter systemic responses, it can also exert direct effects on individual cells, in particular, endothelial cells. It is interesting to note that Vimentin is also regulated by hypoxia and undergoes redistribution under hypoxic conditions. A study investigating the mechanism by which hypoxia precipitated the redistribution of Vimentin focused on the phosphorylation of Vimentin and its interaction with heat shock protein 27 (HSP27). The investigators observed that HSP27 overexpression increased total Vimentin levels in endothelial cells, and both hypoxia and HSP27 overexpression appeared to increase the amount of Vimentin that coimmunoprecipitated with HSP27. It was thus hypothesized that HSP27 may target a regulatory subunit of Vimentin. Under hypoxic conditions, cells appear to undergo changes in biomechanical properties, which would prepare for an adaptive response 
to strengthen tissue and thereby protect it against injury. Some have suggested that these changes bolster adhesive properties and barrier integrity under hypoxic and other possible conditions as well.

Coincidently, the mediator of hypoxic responses within cells, the activity of hypoxia-inducible factor-1 activity (HIF-1) is regulated by copper. Previous studies have shown that pressure overload results in copper decrease in the heart. This is also associated with the inhibition of angiogenesis, as well as the transition from cardiac hypertrophy to heart failure in a mouse model ${ }^{159}$. Copper supplementation at physiologically relevant levels reversed heart hypertrophy in the presence of pressure overload, and this reversal effect was through CCS-mediated HIF-1 activation of VEGF expression and angiogenesis. Copper chelation decreases transcriptional activity of HIF-1, and siRNA targeting the $\alpha$ subunit of HIF-1 blocked copperinduced stimulation of VEGF. Cu is capable of stabilizing HIF- $1 \alpha$ by inhibiting prolyl hydroxylases, and thereby preventing its degradation. While the role of Vimentin in cardiac myocytes has yet to be elucidated, we observed that after PE treatment, Vimentin levels decreased and with $\mathrm{Cu}$ treatment, Vimentin levels increased. We hypothesize that in cardiac myocytes, Vimentin acts as a molecular switch. Upon the addition of PE, the decrease in Vimentin acts as a molecular switch, switching signaling pathways to hypertrophic pathways and resulting in an increase in cardiac myocyte size. Conversely, the Cu treatment causes an increase in VEGFR-1 signaling and Vimentin and thereby switches signaling pathways from hypertrophic, to regression of hypertrophy. 


\section{1-6. HYPOTHESIS}

We hypothesize that $\mathrm{Cu}$ supplementation generates a change in VEGF receptor distribution and the subsequent signaling pathways, leading to the reversal of cardiac hypertrophy. More specifically, we hypothesize that VEGFR-1 is associated with PKG-1 and their association is involved in $\mathrm{Cu}$ induced regression of cardiomyocyte hypertrophy. 


\section{MATERIALS, INSTRUMENTS \& METHODS}

\section{A. MATERIALS AND VENDORS:}

The first 1-13 were used for the isolation of neonatal rat cardiac myocytes.

1. Hanks' balanced salt solution (HBSS): Sterile, calcium and magnesium-free HBSS was purchased from Worthington Biochemical Corporation, Catalog \# LKL003210, $\mathrm{pH} 7.4$, and was stored at $4^{\circ} \mathrm{C}$. This solution was used to dissolve trypsin and trypsin inhibitor as indicated, in addition to incubation of minced neonatal rat hearts on the first day. For washing purposes, HBSS was purchased from GIBCO, Catalog \# 14185. This solution was also calcium, magnesium and magnesium sulphate-free. The $10 \mathrm{X}$ solution was diluted to $1 \mathrm{X}$ and the $\mathrm{pH}$ was adjusted to 7.4 .

2. L-15 media powder: This powder was purchased from Worthington Biochemical Corporation. Catalog \# LK003250 and stored at $4^{\circ} \mathrm{C}$. To prepare the solution, one pouch of the L-15 powder was placed into a $1 \mathrm{~L}$ glass beaker. $900 \mathrm{~mL}$ of double distilled water ( $d d H 20)$ was used to reconstitute the powder, and the pouch was also rinsed out to remove all traces of the powder. The solution was mixed with a magnetic stir bar, and the $\mathrm{pH}$ adjusted to 7.2. The volume was brought to $1000 \mathrm{~mL}$ with ddH2O. After filter sterilization, the solution was stored at $4^{\circ} \mathrm{C}$ until needed.

3. Trypsin: This was purchased from Worthington Biochemical Corporation. Catalog \# LK003235. The powder was stored at $4^{\circ} \mathrm{C} .2 \mathrm{~mL}$ of sterile HBSS was used to dissolve the powder. The solution was prepared freshly for each experiment. 
4. Trypsin inhibitor: This was purchased from Worthington Biochemical Corportation. Catalog \# LK003225. The powder was stored at $4^{\circ} \mathrm{C} .1 \mathrm{~mL}$ of sterile HBSS solution was used to dissolve the powder. This solution was prepared fresh for each experiment.

5. Collagenase: This was purchased from Worthington Biochemical Corporation. Catalog \# LK003245. The powder was stored at $4^{\circ} \mathrm{C} .5 \mathrm{~mL}$ of sterile L-15 media was used to reconstitute the powder. The solution was prepared fresh for each experiment.

6. DMEM/F12: Dulbecco's Modified Eagle Medium: Nutrient Mixture F-12 (Ham) (1:1) powder was purchased from $G I B C O$, Catalog \# 12500-062 and was stored at $4^{\circ} \mathrm{C}$. One pouch of DMEM/F12 powder was placed into a $1 \mathrm{~L}$ glass beaker. $800 \mathrm{~mL}$ of $\mathrm{ddH} 2 \mathrm{O}$ was used to dissolve the powder, as well as rinse out the pouch of any remaining powder. 2.38 grams of HEPES (4-(2-hydroxyethyl)-1piperazineethanesulfonic acid, Sigma, Catalog \# H0891) was added to the solution. The solution was mixed with a magnetic stir bar, and the $\mathrm{pH}$ adjusted to 7.4 . The final volume was brought to $1000 \mathrm{~mL}$ with ddH2O. After filter sterilization, the solution was stored at $4^{\circ} \mathrm{C}$ until needed.

7. DMEM: Dulbecco's Modified Eagle Medium. This powder was purchased from Sigma-Aldrich, Catalog \# D7777 and stored at $4^{\circ} \mathrm{C}$. To reconstitute, one pouch of the DMEM powder was placed into a $1 \mathrm{~L}$ glass beaker. The powder and the remaining contents in the pouch were dissolved with $800 \mathrm{~mL}$ of ddH2O. 3.7 grams of sodium bicarbonate (NaHCO3) was added to the solution. The solution was mixed with a magnetic stir bar and the $\mathrm{pH}$ was adjusted to 7.2. The final volume was brought to 
$1000 \mathrm{~mL}$ using ddH2O. After filter sterilization, the solution was stored at $4^{\circ} \mathrm{C}$ until needed.

8. Cell strainers: These were purchased from Worthington Biochemical Corporation. Catalog \# LK003265 and was used to remove tissue debris during the cell isolation process. They were stored at room temperature.

9. Antibiotic-antimycotic solution: This solution was purchased from Cellgro, Catalog \#30-004-CI. The content includes $10,000 \mathrm{U}$ penicillin, $10,000 \mu \mathrm{g} / \mathrm{mL}$ streptomycin and $25 \mu \mathrm{g} / \mathrm{mL}$ amphotericin B. $1 \%$ of this solution was used in working media (1 mL of solution per $100 \mathrm{~mL}$ of media). This solution was stored at $-20^{\circ} \mathrm{C}$, and thawed prior to use.

10. Phosphate Buffered Saline (PBS): 1X PBS (1 L) was composed of 8 grams $\mathrm{NaCl}$, 0.2 grams $\mathrm{KCl}, 1.44$ grams $\mathrm{Na} 2 \mathrm{HPO} 4,0.24$ grams $\mathrm{KH} 2 \mathrm{PO} 4$. The $\mathrm{pH}$ of the solution was adjusted to 7.4 , filter sterilized and stored at $4{ }^{\circ} \mathrm{C}$ until needed.

11. Gelatin: Gelatin from porcine skin (type A) was purchased from Sigma-Aldrich, Catalog \# G-2500. Gelatin (2.5\%) was prepared by adding 2.5 grams gelatin powder to $100 \mathrm{~mL}$ of PBS. The solution was autoclaved to dissolve and sterilize the solution. Sterilized gelatin was stored at $4^{\circ} \mathrm{C}$ until needed. Prior to use, the solution as warmed up in a $37^{\circ} \mathrm{C}$ water bath for 15 minutes. $1 \%$ of the gelatin solution was used in working media ( $1 \mathrm{~mL}$ of $2.5 \%$ gelatin solution per $24 \mathrm{~mL}$ PBS).

12. Fetal Bovine Serum (FBS)-premium select: This was purchased from Atlanta Biologicals, Catalog \# S11550. FBS was aliquoted and stored in $-20^{\circ} \mathrm{C} .10 \%$ of FBS was used in working media ( $10 \mathrm{~mL}$ serum was added per $100 \mathrm{~mL}$ media). 
13. Penicillin-Streptomycin: This solution was a $100 \mathrm{X}$ dual antibiotic solution. It was purchased from MP Biomedicals, Catalog \# 1670046. It contained 5,000 IU/mL penicillin and $5 \mathrm{mg} / \mathrm{mL}$ streptomycin. $1 \%$ of this solution was used in working media (1 $\mathrm{mL}$ antibiotic solution per $100 \mathrm{~mL}$ media). It was stored at $-20^{\circ} \mathrm{C}$ and thawed in a $37^{\circ} \mathrm{C}$ water bath prior to use.

14. 5-Bromo-2'-deoxyuridine (BrdU): This powder was purchased from Sigma Aldrich, Catalog \# B5002. BrdU (10 mM) was prepared as a stock solution by adding $307 \mathrm{mg}$ BrdU powder to $100 \mathrm{~mL}$ of ddH2O. The solution was filter sterilized, and aliquoted to $10 \mathrm{~mL}$ and stored at $-20^{\circ} \mathrm{C}$ prior to use. The reason for the usage of $\mathrm{BrdU}$ is that it is a thymadine analog, and selectively incorporates into cellular DNA during S-phase. It was used to inhibit the growth of fibroblasts and other proliferative cells in the primary cultures of neonatal rat cardiac myocytes.

15. Disposable sterile bottle-top filters: Filters were purchased from Corning, Catalog \# 43026. It has a funnel volume of $150 \mathrm{~mL}$ and the pore size of the cellulose acetate membrane is $0.22 \mu \mathrm{m}$. They screw onto the mouths of the cell culture bottles and were used to sterilize all solutions used in cell culture. They were stored at room temperature.

16. Copper sulfate (CuSO4): Copper sulfate was purchased from Sigma-Aldrich, Catalog \# C1297. CuSO4 $(10 \mathrm{mM})$ was prepared as a stock solution. $8 \mathrm{mg} \mathrm{CuSO} 4$ powder was added to $5 \mathrm{~mL}$ of ddH2O. The solution was filter sterilized, then aliquoted to $100 \mu \mathrm{L}$ and stored at $-20^{\circ} \mathrm{C}$ prior to use.

17. Phenylephrine (PE): Phenylephrine was purchased from Acros Organics, Catalog \# 61-76-7. PE $(10 \mathrm{mM})$ was prepared as a stock solution. $10 \mathrm{mg}$ PE powder was added 
to $5 \mathrm{~mL}$ ddH2O. The solution was filter sterilized, then aliquoted to $100 \mu \mathrm{L}$ and stored at $-20^{\circ} \mathrm{C}$ prior to use.

18. 0.25\% Trypsin-EDTA.4Na solution (1X): This was purchased from GIBCO, Catalog \# 25200-056. The content containes $2.5 \mathrm{~g} / \mathrm{L}$ of trypsin and $0.38 \mathrm{~g} / \mathrm{L}$ EDTA. $4 \mathrm{Na}$ in $\mathrm{HBSS}$ solution without $\mathrm{CaCl} 2, \mathrm{MgCl} 2 \cdot 6 \mathrm{H} 2 \mathrm{O}$ and $\mathrm{MgSO} 4 \cdot 7 \mathrm{H} 2 \mathrm{O}$. It also contained phenol red as a pH indicator. This solution was used to detach cells in cultures.

19. Lysis buffer: Two different kinds of lysis buffer were used. In the cultures of neonatal rat cardiac myocytes, the content of the lysis buffer included $20 \mathrm{mM}$ Tris$\mathrm{HCl}(\mathrm{pH} 7.4), 150 \mathrm{mM} \mathrm{NaCl}, 1 \%$ Triton-X, $10 \%$ glycerol. Phenylmethylsulfonyl fluoride (PMSF) at a final concentration of $1 \mathrm{mM}$ and $1 \%$ protease inhibitor cocktail (Sigma-Aldrich, Catalog \# P8340) were freshly added prior to use. In the cultures of human cardiac myocytes, the lysis buffer included $30 \mathrm{mM}$ MOPS, pH 7, $0.15 \mathrm{M}$ $\mathrm{NaCl}, 1 \mathrm{mM}$ NaVO4, $5 \mathrm{mM} \mathrm{NaF}, 1 \mathrm{mM}$ EDTA, 1\% NP-40 and $1 \mathrm{mM}$ PMSF and 1\% protease inhibitor cocktail were freshly added prior to use.

20. Human Cardiac Myocytes (HCM), embryonic: Cardiac myocytes were purchased from ScienCell, Catalog \# 6200. These cells were grown in several flasks, collected and then stored in liquid nitrogen for use in experiments.

21. Cardiac Myocyte Medium (CMM): Medium was purchased from ScienCell, Catalog \# 6201. CMM arrived filter sterilized and incomplete, but with reagents needed for completion kept separate. Prior to usage, FBS and antibiotic solution shipped with CMM was added to the medium. Medium was stored at $4{ }^{\circ} \mathrm{C}$ and warmed up in a $37^{\circ} \mathrm{C}$ bath for 30 minutes prior to usage. 
22. Poly-L-Lysine (PLL): PLL ( $1 \mathrm{mg} / \mathrm{mL}$ ) was purchased from ScienCell, Catalog \# 0403. PLL was used to pre-treat cell culture flasks to promote cell adhesion in cultures. The company recommended $15 \mu \mathrm{L}$ of PLL per $1 \mu \mathrm{L}$ of ddH2O, however some optimization was required depending on the flasks used for cell culture. Flasks must be treated for at least 1 hour prior to seeding cells, or were treated overnight. After treatment with PLL, flasks were rinsed with PBS twice, and not allowed to dry out prior to cell seeding. PLL was stored at $-20^{\circ} \mathrm{C}$ prior to usage, and was warmed in a $37^{\circ} \mathrm{C}$ bath briefly when needed.

23. Protein assay kit: This was purchased from BioRad, Catalog \# 500-0006 and was used according to the manufacturer's instructions.

24. Protein G Sepharose beads: These beads were purchased from Sigma-Aldrich, Catalog \# P3296-5ML. These beads were washed 3X with lysis buffer, centrifuging between each wash to collect the beads in the bottom of a microcentrifuge tube. After the last wash, a $50 / 50$ solution of beads/lysis buffer was made. $20-40 \mu \mathrm{L}$ of this slurry solution was added to sample tubes during co-immunoprecipitation experiments.

25. Agarose-conjugated cGMP beads: These beads were purchased from Santa Cruz Biotechnology, Catalog \# sc10335ac. $2 \mu \mathrm{g}$ of antibody was added to cell lysates and incubated overnight for immunoprecipitation. The reason for using agaroseconjugates is to minimize signal disturbance during subsequent LC/MS-MS analysis.

26. Agarose-conjugated Flt-1 beads: These beads were purchased from Santa Cruz Biotechnology, Catalog \# sc316ac. $2 \mu \mathrm{g}$ of antibody was added to cell lysates and 
incubated overnight for immunoprecipitation. The reason for using agaroseconjugates is to minimize signal disturbance during subsequent LC/MS-MS analysis.

27. Vimentin antibody for Western blot: This antibody was purchased from Cell Signaling, Catalog \# 3932S. Vimentin antibody used for Western blot was incubated with the membrane overnight with diluted antibody (1:500-1:1000) in 5\% w/v BSA, $1 \mathrm{X}$ TBS, $0.1 \%$ Tween- 20 at $4^{\circ} \mathrm{C}$ with gentle shaking.

28. Vimentin antibody for Immunoprecipitation: This antibody was purchased from Cell Signaling, Catalog \# 3390. Vimentin antibody was added to lysate slurry 1:50 and incubated overnight at $4^{\circ} \mathrm{C}$ under rotation.

29. Flt-1 antibody for Western blot: This antibody was purchased from Santa Cruz Biotechnology, Catalog \# sc316. It is critical for this experiment that the membrane not be blocked with non-fat milk, instead BSA must be used or no band will appear. Antibody dilution ranged from 1:500-1:1000.

30. Detergent removal spin columns: These columns were purchased from Thermo Scientific, Catalog \# 87777 and were used according to the manufacturer's instructions.

\section{B. Methods}

1. Primary culture

Primary cultures of neonatal rat cardiac myocytes were established according to a previously published procedure but with some modifications (Hoffmann, et al., 1995). The hearts of 1-3 day old Sprague-Dawley rats were isolated, minced on ice and incubated overnight at $4^{\circ} \mathrm{C}$ with $50 \mu \mathrm{g} / \mathrm{mL}$ trypsin (Worthington Biochemical Corporation, Lakewood, NJ) in $20 \mathrm{~mL}$ calcium and magnesium-free Hanks Balanced 
Salt Solution (HBSS, Life Technologies Ltd, Carlsbad, CA). After 16 hours incubation, soybean trypsin inhibitor was added at a final concentration of $100 \mathrm{mg} / \mathrm{mL}$ for 30 minutes. During this time, the flask was placed in an incubator, $37^{\circ} \mathrm{C}$ and the tissue was digested with collagenase (Worthington Biochemical Corporation) in $5 \mathrm{~mL} \mathrm{L-15}$ media (Worthington Biochemical Corporation) at $37^{\circ} \mathrm{C}$ for 70 minutes. After pipetting the cells gently, and filtering through a cell strainer, the solution was centrifuged at $1000 \mathrm{rpm}$ for 5 minutes; this was to remove cells such as endothelial cells and fibroblasts. The supernatant was removed, and the pellet of cells was resuspended in DMEM/Ham's nutrient mixture F12 (1:1 vol/vol, Life Technologies Ltd) supplemented with antibiotics (100 U/mL penicillin and $100 \mu \mathrm{g} / \mathrm{mL}$ streptomycin, MP Biomedicals, LLC, Solon, OH), $10 \%$ fetal bovine serum (FBS, Life Technologies Ltd) and $0.1 \mathrm{mM}$ 5-bromo-2'deoxyuridine (BrdU, Sigma-Aldrich, St. Louis, MO). Fibroblasts were removed by preincubation for 2 hours at $37^{\circ} \mathrm{C}$ in the cultures. Cardiac myocytes in suspension were collected by centrifugation, further purifying the cardiac myocytes. The cardiac myocytes were seeded in $1 \%$ gelatin-coated plates containing DMEM/F12 media supplemented with FBS, BrdU and antibiotics for 24 hours before subsequent experiments. Myocytes were seeded at a density of $1.5 \times 10^{6} / 10 \mathrm{~cm}$ culture dish; $6 \times 10^{5} / 60$ $\mathrm{mm}$ culture dish, $2 \times 10^{5} / 35 \mathrm{~mm}$ culture dish. After 4 hours the media was changed to serum-free Dulbecco's modified Eagle's media (DMEM, Gibco, Gaithersburg, MD) supplemented with BrdU and antibiotics. Beating cardiac myocytes were observed under routine microscopy. Unless $90 \%$ of the cells were beating, the preparation was not used. The purity of the cultured cardiac myocytes was examined by staining with anti- $\alpha$ sarcomeric actin antibody (Sigma-Aldrich) as described below. 
At the end of each experiment (48 hours PE treatment and 24 hours with $\mathrm{Cu}$ ), the purity of the cardiac myocytes was determined using flow cytometry. The purity was more than 90\%. Briefly, cells were trypsinized, washed with PBS and fixed in $75 \%$ ethanol at $4^{\circ} \mathrm{C}$ for at least 18 hours. Primary anti-a-sarcomeric actin antibody (SigmaAldrich) was diluted in PBS and incubated at room temperature for 1 hour. Secondary fluorescein-conjugated goat anti-mouse IgM antibody (FITC, Southern Biotech, Temecula, CA) was used for immunofluorescence and incubated for 30 minutes at room temperature. The fluorescence distribution from 30,000 cells was captured and recorded using a CellQuest Acquisition program (Becton \& Dickinson, Franklin Lakes, NJ). Data were analyzed using FlowJo software (Tree Star, Inc., Ashland, OR) and the perentage of the $\alpha$-sarcomeric actin labeled cardiac myocytes in the population was calculated.

\section{Experimental procedure}

Cell hypertrophy was induced by phenylephrine (Sigma-Aldrich) at a final concentration of $100 \mu \mathrm{M}$ for 48 hours in serum-free media, following a previously published procedure ${ }^{173}$. At the end of the 48 hour incubation, $\mathrm{Cu}$ in the form of copper sulfate (CuSO4) was added directly to the cultures at a final concentration of $5 \mu \mathrm{M}$ for an additional 24 hours. At the end of the treatment, or 72 hours, the media were collected for VEGF secretion analysis. The cells were collected by trypsinization, suspended in PBS buffer and counted using a hemocytometer. Protein content was measured using a Bradford method (Bio-Rad, Hercules, CA) and normalized by the cell number. For flow cytometry analysis of VEGF receptors, the harvested cells were subjected to fixation and incubation with different markers before analysis as described below. 
To assess the role of PKG-1 in the $\mathrm{Cu}$-induced regression of cell hypertrophy, we used the PKG-1 $\alpha$ antagonist, (Rp-8-CPT-cGMPS) (Sigma), at a final concentration of 0.5 $\mu \mathrm{M}$ in cultures. The antagonist was added to the cultures 1 hour prior to the addition of $\mathrm{Cu}$ in cultures. In VEGFR-2 siRNA-induced regression of cell hypertrophy, the same concentration of VEGF antibody and PKG antagonist was applied.

\section{Protein assay}

Protein concentrations were measured by the Bradford method using a commercially available assay kit according to the manufacturer's instructions (Bio-Rad, Hercules, CA). The absorbance at $595 \mathrm{~nm}$ was measured using a Beckman CU 650 spectrophotometer (Pegasus Scientific, Frederick, MD). Protein content per cell was determined by dividing the total amount of protein by the cell number and the final concentration was expressed as micrograms per $10^{6}$ cells.

\section{Cell size determination}

Cell volume was determined using a Lovins microslide field finder (Fisher Scientific, Pittsburgh, PA) following the procedure described previously ${ }^{174}$. Briefly, cardiac myocytes in cultures were trypsinized, suspended in PBS and loaded onto a microslide field finder. The diameters of approximately 150 cells from each group were assessed and recorded. The cell volume was calculated using the volume of a sphere: $V=(4 / 3) \pi r^{3}$ where $\mathrm{V}=$ cell volume, $\pi=3.14$ and $\mathrm{r}=$ radius.

In addition, cell size was also determined by flow cytometry. Briefly, cells were trypsinized, suspended in PBS and directly passed through the flow cytometer. For each sample, 30,000 cells were recorded and analyzed through the forward scatter light (FSC), 
which is proportional to cell size. The data were quantitatively analyzed sing the FlowJo software.

\section{Immunocytochemistry}

The examine morphological changes of cardiac myocytes in cultures after treatment with PE and $\mathrm{Cu}$, cells were cultured in an 8-well chamber slide and treated as described in experimental procedure. After $\mathrm{Cu}$ treatment for 24 hours, cells were fixed in $4 \%$ paraformaldehyde in PBS. After fixation, the culture chamber was incubated with monoclonal anti- $\alpha$-sarcoeric-actin antibody (Sigma-Aldrich) followed by incubation with FITC-conjugated goat anti-mouse IgM. Fluorescence was visualized by a phase contrast microscope (Nikon Instruments, Melville, NY). Images were acquired by a Nikon digital camera DXM 1200 (Nikon Instruments) using a Nikon ACT-1 software.

6. Double immunochemistry staining

To examine the alterations of the distribution of VEGF receptors of the membrane of cardiac myocytes after treatment with $\mathrm{PE}$ and/or $\mathrm{Cu}$, cells were cultured in 8-well chamber slides and treated as described in the experimental procedure. After $\mathrm{Cu}$ treatment for 24 hours, cells were fixed in $100 \%$ methanol. After fixation, the culture chamber was incubated with polyclonal anti-VEGFR-1 antibody (Santa Cruz) followed by incubation with Alexa Fluor 488 conjugated goat anti-rabbit IgG. After washing the culture chamber was incubated with monoclonal antiVEGFR-2 antibody (Santa Cruz) followed by incubation with Alexa Fluor 594 conjugated goat anti-mouse IgG.

Fluorescence was visualized by a phase contrast microscope (Nikon Instruments, Melville, NY). Images were acquired by a Nikon camera DXM 1200 (Nikon Instruments) using a Nikon ACT-1 software. 
7. Flow cytometric analysis of VEGF receptors

Quantitative analysis of VEGF receptors in cardiac myocytes under different treatments was done by flow cytometric assay. Briefly, cells were trypsinized, washed with PBS and fixed in $75 \%$ ethanol at $4^{\circ} \mathrm{C}$ for at least 18 hours. Immunofluorescence staining with an Alexa Fluor 488 conjugated antibody to VEGF-1 (1:50, Santa Cruz) and Alexa Fluor 594 conjugated antibody to VEGFR-2 (1:50, Santa Cruz) were performed on fixed cardiac myocytes. Receptor expression was determined by the intensity of the fluorescence using the software FlowJo based on the data collected from 30,000 cells per sample. The data were plotted in two-dimensional fluorescence intensity versus cell number.

8. SiRNA

siRNA targeting rat VEGFR-2 and negative mismatched control siRNA were purchased from Ambion, Inc. (Applied Biosystems, Cambridge, MA siRNA ID\#: S130202). The siRNA sequences for VEGFR-2 were (from 5' to 3'): GGGUAUCACUCAGACGAGAtt (sense); UGUCGUCUGAGUGAUACCCag (antisense). The siRNA sequences for VEGFR-1 were (from 5' to 3'): GAAGCGGUCUUCUUCCGAAtt (sense); UUCGGAAGAAGACCGCUUCag (antisense). The optimal transfection efficiency was determined from our preliminary study testing the range from $5-20 \mathrm{nM}$, and at the selected level of siRNA with maximum silencing effect with minimal toxicity. After PE treatment, cardiac myocytes were transfected for 4 hours with $10 \mathrm{nM}$ annealed siRNA targeting rat VEGFR-1 or VEGFR-2 or neative mismatched siRNA in antibiotic-free media as antibiotics can cause cell death under these conditions. Lipofectamine 2000 (Invitrogen) was used as the transfection 
reagent according to the manufacturer's instructions. After 4 hours of incubation, cardiac myocytes were changed to the original serum-free media with antibiotics and PE was added again for hypertrophic groups. At 48 hours post-transfection, cells were trypisinzed and collected. The silencing effect of siRNA was examined by flow cytometry 24 hours after siRNA transfection. From preliminary results, VEGFR-2 level was increased after PE treatment in cardiac myocytes compared with control, and $\mathrm{Cu}$ addition lowered the protein expression. To investigate the effect of siRNA, PE treated cells were used as a positive control. siRNA-treated cardiac myocytes decreased the protein expression of VEGFR-2 in PE-treated cells, which is very similar to the effect of $\mathrm{Cu}$.

9. cGMP-dependent protein kinase activity

cGMP-dependent protein kinase G (PKG)-1 activity was assayed by coloimetric analysis with a non-radioisotopic kit ( $M B L$, Woburn, MA). The assay was performed 30 minutes after treatment with $\mathrm{Cu}$ in the experiment of VEGFR-1 siRNA-blocked regression of hypertrophic cardiac myocytes. Each sample was measured in duplicate carefully following the manufacturer's instructions.

10. Human Cardiac Myocytes Cell Culture Experimental procedure

T-75 flasks were pre-treated with PLL for either 1 hour or overnight, then washed 2 times with PBS prior to cell plating. HCM were obtained, grown and maintained in CMM. When the cell density is low ( $<50 \%$ confluent) media may be changed every other day, however for higher density cell populations, media must be changed daily.

Cardiac myocyte hypertrophy was induced by PE at a final concentration of $100 \mu \mathrm{M}$ for 48 hours. Copper $(\mathrm{Cu})$ in the form of copper sulfate $\left(\mathrm{CuSO}_{4}\right)$ was added directly to 
the cultures at a final concentration of $5 \mu \mathrm{M}$ for an additional 24 hours in the concomitant presence of PE. At the conclusion of $\mathrm{Cu}$ treatment, cells were washed with phosphate buffered saline (PBS), and collected by trypsinization and centrifuged at 2000 RPM for 5 minutes at $4^{\circ} \mathrm{C}$. Cells were washed with PBS to remove trypsin and centrifuged again under the aforementioned conditions and the PBS was removed. If cells were not used immediately, they were stored in $-80^{\circ} \mathrm{C}$ until needed.

11. Western Blot Procedure

Cells were collected as previously described using trypsinization and centrifugation. The pellet was washed $2 \mathrm{X}$ with ice-cold PBS, after the final wash, remove as much PBS as possible without disturbing the pellet. Lysis buffer: $30 \mathrm{mM}$ MOPS, $\mathrm{pH} 7.0,0.15 \mathrm{M}$ $\mathrm{NaCl}, 1 \mathrm{mM} \mathrm{Na} 3 \mathrm{VO} 4,5 \mathrm{mM} \mathrm{NaF}, 1 \mathrm{mM}$ EDTA, $1 \% \mathrm{NP}-40$ and protease inhibitors were added to the pellet. The tubes were always kept on ice and handling kept to a minimum to keep the contents and tube as cool as possible.

Cell lysis was achieved by using a cell sonicator. The sonicator was set to 3-5. The cells were sonicated for 1 second, and then rest on ice for 10 seconds. This was repeated 2 more times. After, the tubes were placed under constant agitation or in head-over-tail rotor in $4^{\circ} \mathrm{C}$ for $30-60$ minutes. In order to pellet non-soluble material, the tubes underwent centrifugation at $12,000 \mathrm{x}$ for 15 minutes at $4{ }^{\circ} \mathrm{C}$. The supernatant was carefully removed and transfered to a fresh tube on ice.

Protein concentration was determined as previously described using the Bradford method. Proteins were reduced and denatured by the addition of $\beta$-mercaptoethanol to the loading buffer, and samples were boiled at $95^{\circ} \mathrm{C}$ for 10 minutes. 
Samples were carefully loaded into pre-cast gels and the gel was placed on ice or in $4^{\circ} \mathrm{C} .60$ volts was applied to the gel for 30 minutes, then $120 \mathrm{~V}$ until the bands are roughly 1 centimeter from the bottom of the gel.

Following SDS-PAGE, the gel was prepared to undergo electroblotting using a nitrocellulose membrane (GE Healthcare) at 100 volts for 150 minutes. The membrane was then washed with $1 \mathrm{X}$ TBST and blocked with $5 \%$ w/v BSA, $1 \mathrm{X}$ TBS, $0.1 \%$ Tween20 for 1 hour at room temperature. The membrane was incubated with diluted antibody. Vimentin was detected by incubation overnight at $4^{\circ} \mathrm{C}$ with 1:500-1:1000 of antiVimentin antibody (Cell Signaling Technology, Inc). PKG-1 was detected by incubation overnight at $4^{\circ} \mathrm{C}$ with 1:500-1:1000 of anti-PKG-1 antibody (Santa Cruz Biotechnology). VEGFR-1 was detected by incubation overnight at $4^{\circ} \mathrm{C}$ with 1:500-1:1000 of antiVEGFR-1 antibody (Santa Cruz Biotechnology).

The following morning, membranes were washed $3 \mathrm{X}$ for 5 minutes each with $1 \mathrm{X}$ TBST, then incubated with the secondary antibody, horseradish peroxidase (HRP) conjugated goat anti-rabbit immunoglobulin G antibody, diluted 1:1,000 in TBS containing $0.5 \%$ Tween-20 at room temperature for 1 hour. Proteins were visualized using enhanced chemiluminescence (ECL) (Amersham, Buckinghamshire, UK), according to the manufacturer's instructions, to visualize HRP-conjugates. 12. Immunoprecipitation

400-500 $\mu \mathrm{g}$ of protein from cell lysates were incubated overnight with anti-VEGFR-1 (Santa Cruz Biotechnology), anti-Vimentin (Cell Signaling Technology) or anti-PKG-1 agarose-conjugated beads (Santa Cruz Biotechnology) antibody at a concentration of 2 $\mathrm{ug} / \mathrm{mL}$ for 16 hours at $4^{\circ} \mathrm{C}$ under rotation. The following morning, 20-40 $\mu \mathrm{L}$ of a Protein 
$\mathrm{G}$ sepharaose beads slurry to the mixture and incubate under rotation at $4^{\circ} \mathrm{C}$ for 4 hours. The samples underwent centrifugation at $1000 \mathrm{Xg}$, and the supernatant was carefully removed, and the bead pellet 3 times with ice-cold PBS. After the final wash, as much of the supernatant was removed as possible.

For co-immunoprecipitation, in which IP is followed by Western blot: lysis buffer was added to the beads and the tube was boiled at $95^{\circ} \mathrm{C}$ for 10 minutes. Samples were centrifuged again, and the supernatant underwent SDS-PAGE for Western blot analysis.

For subsequent analysis via LC/MS-MS, after washing the pellet $3 \mathrm{X}$, to elute the immune complex, capture beads were resuspended in $50 \mu \mathrm{l}$ of $0.1 \mathrm{M}$ glycine, $\mathrm{pH} 2.5$ and incubated with agitation for 10 minutes at $4^{\circ} \mathrm{C}$. The supernatant was collected after centrifugation, run through detergent removal spin columns (Thermo Scientific) according to the manufacturer's instructions, and analyzed using LC/MS-MS.

\section{LC/MS-MS}

After immunoprecipitation, samples were sent off to Dr. Jian Cai for MALDI-TOF analysis. Mass spectrometers contain 3 components: an ionization source, a mass analyzer, in this case, time-of-flight, and a detector. Typically the ionization source comes from a UV light. The mass analyzer functions to separate or resolve ions formed in the ionization source according to the mass/charge $(\mathrm{m} / \mathrm{z})$ ratio. Finally, the detector monitors the ion currents, in the form of mass spectra.

MALDI-TOF, or Matrix Assisted Laser Desorption/Ionization Time of Flight mass spectrometry is a powerful analytical tool for the identification of unknown biomolecules which utilizes a UV-light absorbing matrix. This matrix, which contains the proteins in the sample, is irradiated by a nanosecond laser pulse. The 
majority of the energy is absorbed by the matrix, thereby preventing unwanted fragmentation of the molecules. At the same time, the molecules become ionized, leaving it with a positive charge. The purpose of this is because magnetic force only affects charged particles. The ions then travel through an electric field where they are accelerated, and enter a flight tube. In this tube, they are separated according to their mass/charge ratio, ions with a smaller mass traveling faster than those with a larger mass. Each biomolecule reach the detector at a different time, yielding a distinct signal. This method is highly sensitive, with an accuracy of $0.1-0.01 \%$.

Many things should be taken into consideration for successful experiments when using this procedure. One big source of problems during LC-MS/MS analysis is detergents, which can cause large, unknown peaks. Another source is keratin. In order to minimize potential problems, do as much work as feasible in a laminar flow hood or biological safety cabinet (BSC).

Assume nothing is free of keratin (it probably isn't). That means assuming your pipette tips, Eppindorf tubes, reagent containers, skin—everything is a potential source of keratin. Be mindful of the clothing worn while experimental procedures are being carried out. Wool sweaters are a terrific source of keratin and probably not the best choice of clothing to wear while conducting experiments upstream of mass spectrometry analysis. Even cotton lab coats shed significant amounts of dust, which may contain keratin. If possible, wear a disposable clean-room type lab coat.

Latex gloves contain a significant amount of keratin as well as proteinaceous materials. Nitrile or vinyl gloves are the best choice. Rinse gloves under a stream of water before handling samples. Additionally, working surfaces should be cleaned 
with water and absolute ethanol, and wiped down with clean-room wipes, not dusty, keratin-rich paper towels. Anything that is placed into the BSC should be wiped down thoroughly.

All supplies, whether it be pipette tip boxes or bags of microcentrifuge tubes, should be opened only in the BSC while it is in full operation. Further, detergents are the enemy. Detergents, while important for solubilizing, stabilizing and disaggregating protein complexes will also do nasty things to your sample. It can form adducts with peptides and proteins, and in MS analysis, this will shift $\mathrm{m} / \mathrm{z}$ values, which is bad news. Another big no-no for MS is plasticizers.

Lastly, anything that will come into contact with samples (vials, pipette tips, etc.) should be rinsed with HPLC grade Acetonitrile or high grade methanol if none is available, then with HPLC grade water.

14. Statistical analysis

Data were obtained from three separate experiments and are expressed as means \pm S.E.M. A 2 X2 factorial design was applied to all experiments and the data were analyzed according to the experimental design. After a significant interaction was detected by the analysis of variance (ANOVA) based on the factorial design, the significance of the main effects was further determined by the F-test. The level of significance was considered when $P<0.05$. 


\section{RESULTS}

A. Previous studies have shown that adding physiologically relevant amounts of $\mathrm{Cu}$ to cardiac myocytes in cell cultures can reverse hypertrophy induced by phenylephrine. This effect is blocked by adding a VEGF antibody. This recaptures the in vivo observation that $\mathrm{Cu}$-induced regression of hypertrophy is VEGF-dependent. Additionally, the induction of hypertrophy by phenylephrine can be blocked by using a VEGF antibody. This led us to the question: does VEGF have a duel function, acting to stimulate cell growth leading to hypertrophy as well as reversing hypertrophy?

Cardiac myocyte hypertrophy was induced by PE at a final concentration of $100 \mu \mathrm{M}$ in cultures for 48 hours in serum-free media. Cells were then treated with $\mathrm{CuSO}_{4}$ by direct addition into the cultures at a final concentration of $5 \mu \mathrm{M}$. As seen below in

Figure 1, and much to our surprise, $\mathrm{PE}$ induced VEGF production, but not $\mathrm{Cu}$. $\mathrm{Cu}$ did not affect VEGF, however PE did induce it. This was a rather unexpected observation. 


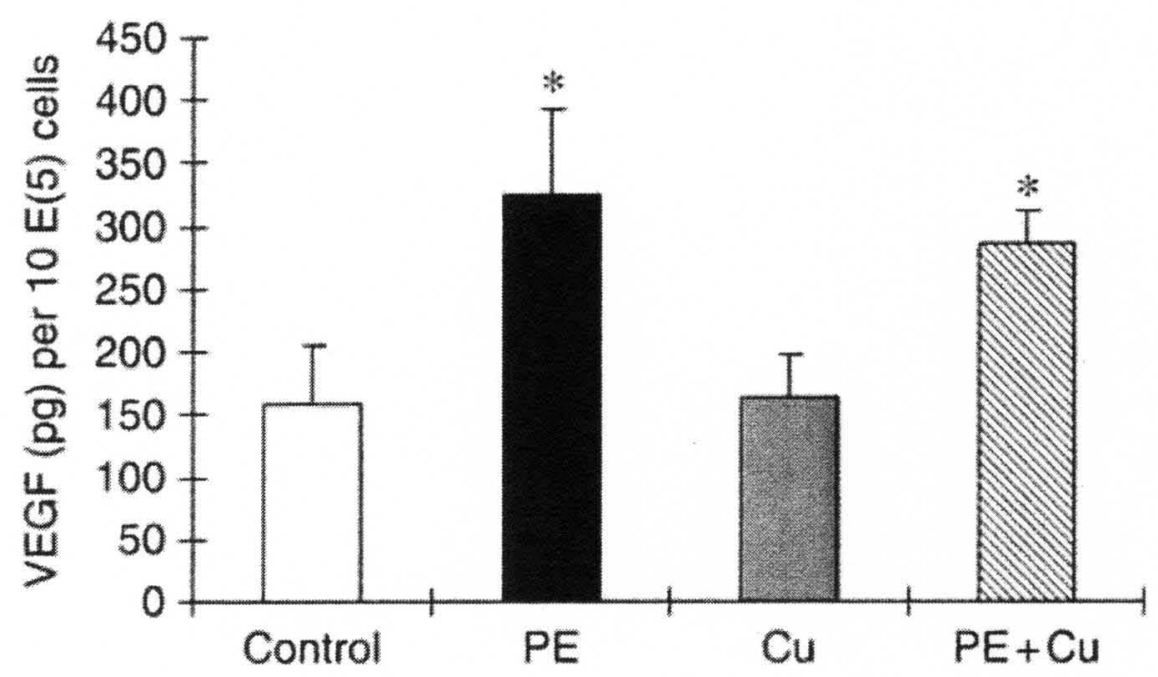

Figure 1. Quantitative analysis of VEGF secretion. Hypertrophy was induced by PE at a final concentration of $100 \mu \mathrm{M}$ for 48 hours in serum-free media. $\mathrm{CuSO}_{4}$ was added directly to the cultures at a final concentration of $5 \mu \mathrm{M}$ for 24 hours. VEGF levels in collected media were determined by a Quantikine VEGF Immunoassay kit. The absorbance of standards and samples was measured spectrophotometrically at $450 \mathrm{~nm}$ with a wavelength of $540 \mathrm{~nm}$ using a microplate reader. VEGF concentrations were calculated (in $\mathrm{pg} / \mathrm{mL}$ ) on the basis of the standard curve. Values are means \pm SEM. * denotes statistically significant from Control group. $n=3$.

\section{B. The effect of Cu treatment on VEGFRs}

The effect of VEGF is mediated through its receptors. VEGF receptors (VEGFRs)

belong to a family of receptors called tyrosine kinases. Upon the binding of VEGF to its receptor, the receptors undergo dimerization and signal transduction. Since the amount of VEGF in the media of cell cultures was unchanged by $\mathrm{Cu}$, the next thing we investigated was the effect that $\mathrm{Cu}$ had upon VEGFRs. There are 2 VEGF receptors in the cardiovascular system, Flt-1, also known as VEGF Receptor 1 (VEGFR-1) and KDR, also known as VEGF Receptor 2 (VEGFR-2). Activation of VEGFR-2 is associated with a mitogenic response, and is also involved in angiogenesis and survival signaling. A 
decoy VEGFR-2 receptor blocked Akt-1 signalling pathway, which is associated with growth and is a critical component in the process of myocardial hypertrophy. VEGFR-1 function was previously enigmatic. Our data suggests it may be associated with the reversal of hypertrophy. A schematic representation ${ }^{175}$ can be seen in Figure 2.

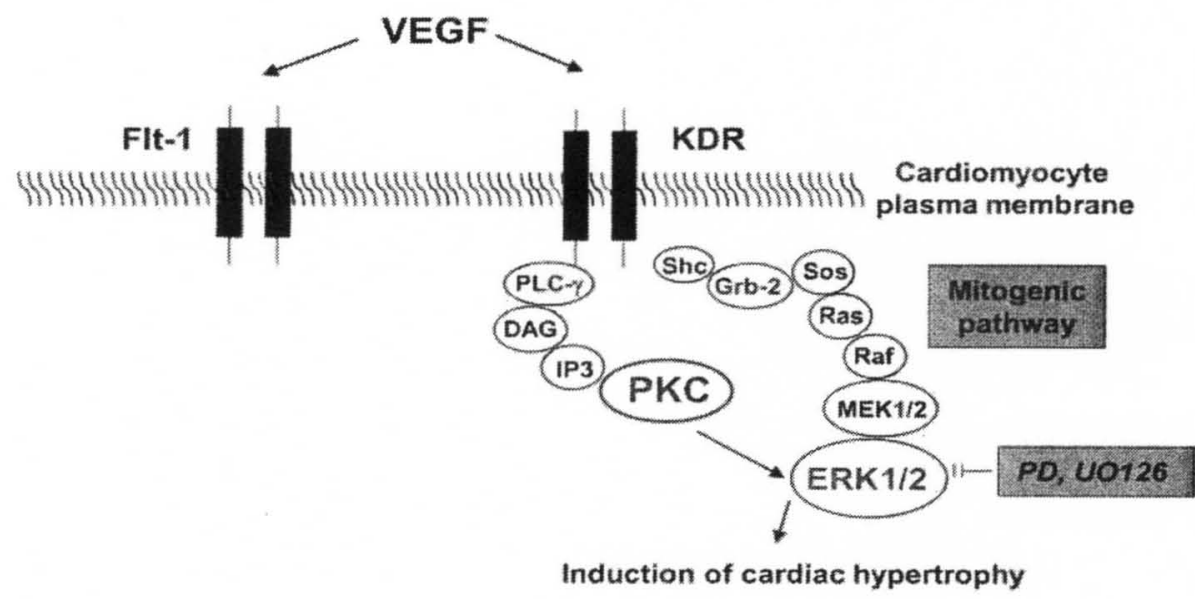

Figure 2. A schematic representation of VEGF receptor signaling in cardiac myocytes. 
The increase in the ratio of VEGFR-1 to VEGFR-2 by $\mathrm{Cu}$ treatment in hypertrophic cardiac myocytes

1. We investigated the effect of $\mathrm{Cu}$ on the density of VEGFRs. As shown below in Figure 3, PE increased the density of both VEGFR-1 (green) and VEGFR-2 (red). VEGFR-1 was not changed by $\mathrm{Cu}$ addition, but markedly reduced that of VEGFR-2 in hypertrophic cardiac myocytes. The merged images of the two fluorescent labeling showed more green color (VEGFR-1) than the orange observed under PE only treatment condition. 


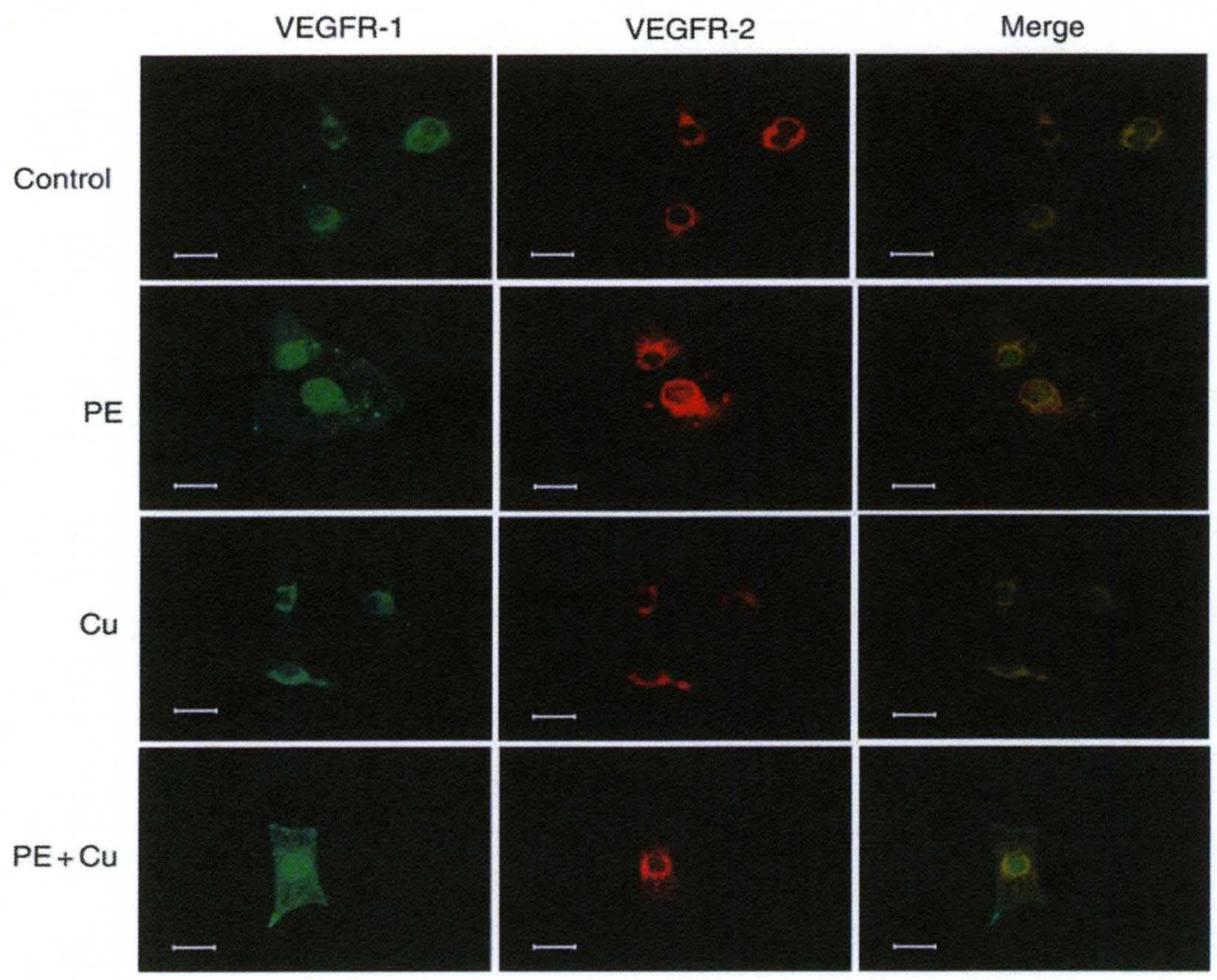

Figure 3. Representative fluorescent microscopic images of cellular density of VEGFR-1 (green) and VEGFR-2 (red) or the combination. Hypertrophy was induced by PE at a final concentration of $100 \mu \mathrm{M}$ for 48 hours in serum-free media. $\mathrm{CuSO}_{4}$ was added directly to the cultures at a final concentration of $5 \mu \mathrm{M}$ for 24 hours. After fixation, cells were incubated with polyclonal anti-VEGFR-1 antibody, followed by Alexa Fluor 488 conjugated goat anti-rabbit IgG. After washing the slide, the culture chamber was incubated with monoclonal antiVEGFR-2 antibody and incubated with Alexa Fluor 594 conjugated goat anti-mouse IgG. Fluorescence was visualized by a fluorescent microscope. Magnification: 400X. 
2. As shown in Figure 4 and 5, we also did quantitative analysis by flow cytometer to confirm the effect of $\mathrm{Cu}$ on the density of VEGFRs. The ratio of VEGFR-1 to VEGFR-2 was significantly increased. Together, with the aforementioned data, this suggests a role for VEGF outside of angiogenesis due to the lack of blood vessels in cell cultures. Because $\mathrm{Cu}$ did not affect the amount of VEGF in cultures, this indicates that it has a direct effect that is mediated through VEGF receptors. And this reversal effect is mediated in part by an increase in the ratio of VEGFR-1:VEGFR-2.
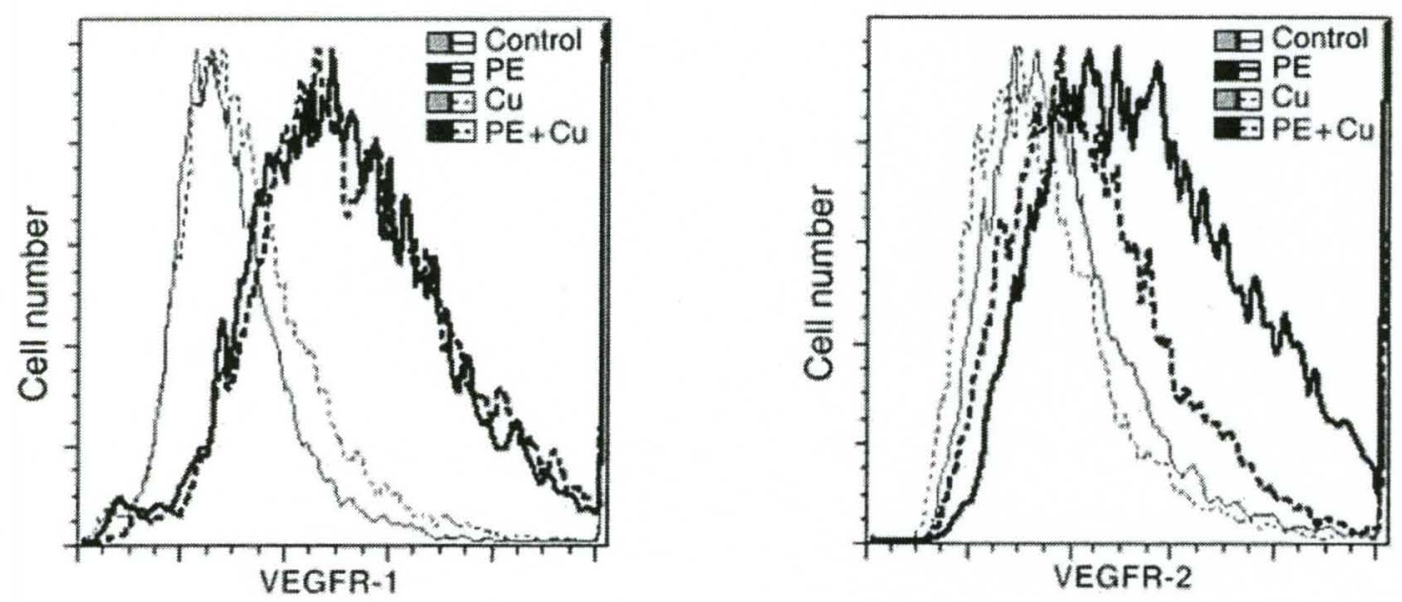

Figure 4. Flow cytometric analysis of VEGFR-1 and VEGFR-2. Hypertrophy was induced by PE at a final concentration of $100 \mu \mathrm{M}$ for 48 hours in serum-free media. $\mathrm{CuSO}_{4}$ was added directly to the cultures at a final concentration of $5 \mu \mathrm{M}$ for 24 hours. Representative histograms show changes in the density of VEGFR-1 and VEGFR-2 under different treatment conditions. The values presented in the figures are means \pm SEM. *Significantly different from Control group $(\mathrm{P}<0.05)$. 

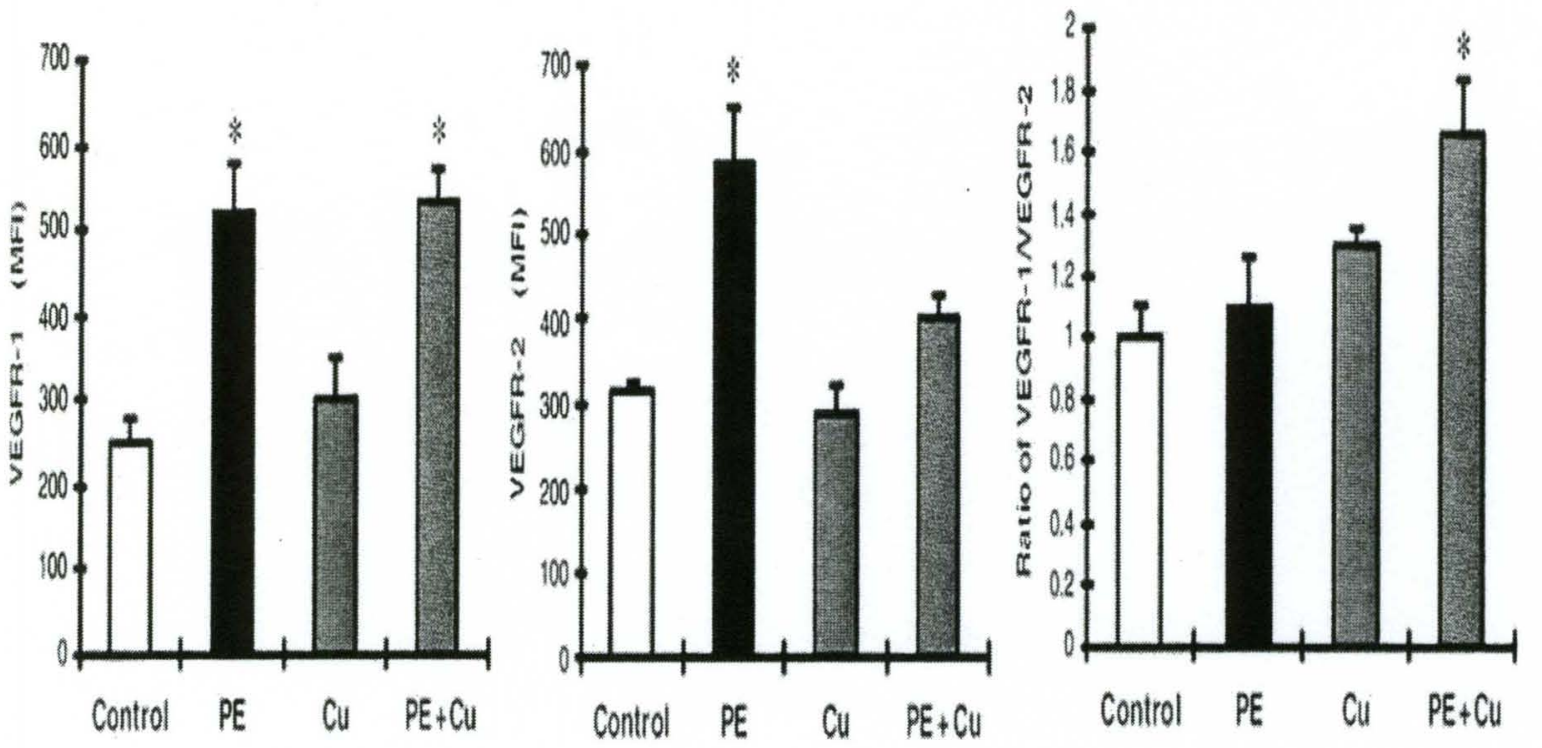

Figure 5. Flow cytometric analysis of VEGFR-1 and VEGFR-2 in cultured neonatal rat cardiac myocytes. Hypertrophy was induced by PE at a final concentration of $100 \mu \mathrm{M}$ for 48 hours in serum-free media. $\mathrm{CuSO}_{4}$ was added directly to the cultures at a final concentration of $5 \mu \mathrm{M}$ for 24 hours. At the end of $\mathrm{Cu}$ treatment, cells were collected, fixed and incubated with Alexa Fluor 488 conjugated antibody of VEGFR-1 and Alexa Fluor 594 conjugated antibody to VEGFR-2. Cells were subjected to flow cytometry analysis and 30,000 cells in each sample were counted. Receptor density was determined by the intensity of fluorescence using the software FlowJo based on the data collection from 30,000 cells per sample. Data were plotted in two-dimensional fluorescence intensity vs. cell number. Each group of data presented were obtained from three independent experiments and each experiment contains a triplicate sample for each treatment. The values presented in the figures are means \pm SEM. *Significantly different from Control group $(\mathrm{P}<$ 0.05 ). MFI $=$ Median Fluorescence Intensity. $n=3$. 


\section{Reversal of PE-induced cardiac myocyte hypertrophy by gene silencing of}

\section{VEGFR-2}

1. Thus far we have observed that the elevation of VEGFR-1:VEGFR-2 has been linked to the $\mathrm{Cu}$-induced regression. This led us to the next question: How does VEGF induce the regression phenomenon? Could it be through VEGFR-1? We hypothesized that in $\mathrm{Cu}$-treated hypetrophic cardiac myocytes, the distribution of VEGFRs would be altered, leading to a swich from cell growth stimulation to regression. To differentiate whether the increase in the ratio of VEGFR1/VEGFR-2 was related to the $\mathrm{Cu}$-induced reversal of cardiomyocyte hypertrophy, we used siRNA targeting VEGFR-2 to increase the ratio of VEGFR1/VEGFR-2. It should be noted that the amount of siRNA used determines the efficacy of the knock down; in this case, the goal was not to completely silence VEGFR-2, but rather mimic the effect of $\mathrm{Cu}$. In figures 6,7 and 8, we show preliminary data of experiments targeting siRNA and flow cytometric analysis of VEGFR-1 and 2 density, then the data plotted as a ratio of VEGFR-1:VEGFR-2. Gene silencing of VEGFR-2 increases the ratio of VEGFR-1 to VEGFR-2 the same as that induced by $\mathrm{Cu}$ treatment, which is also associated with a reversal in cardiac myocyte hypertrophy. The effect is comparable to that induced by $\mathrm{Cu}$ treatment as shown in Figures 9, 10 and 11. 


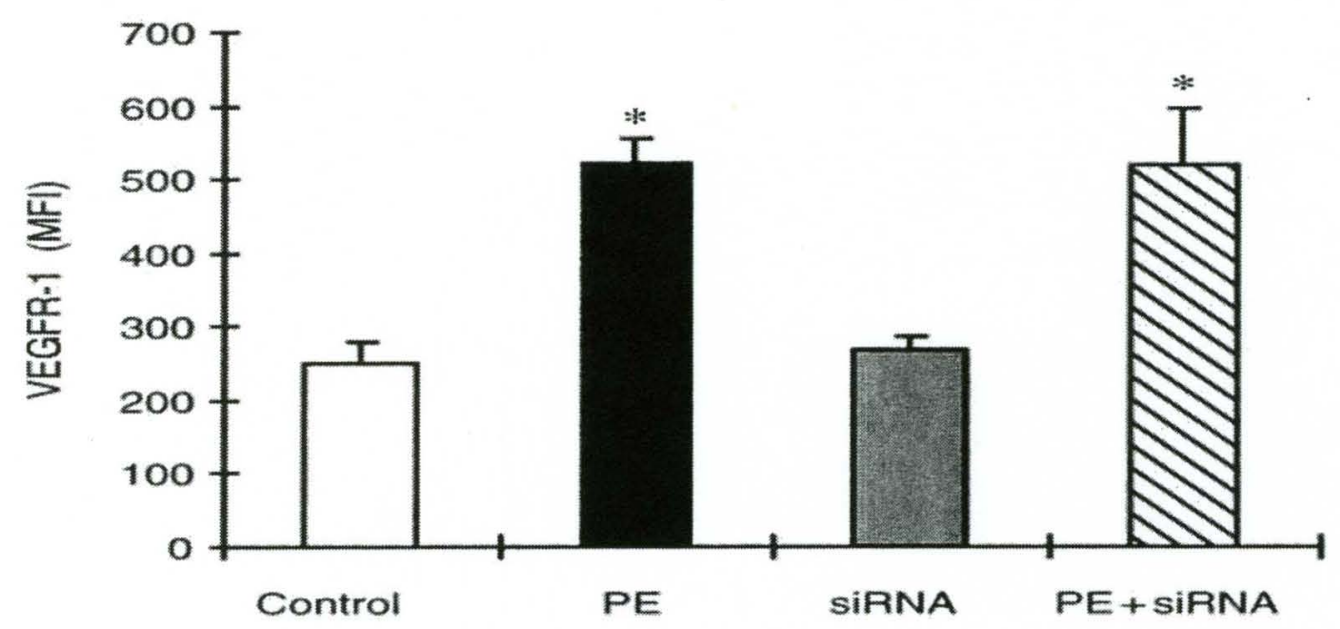

Figure 6. Quantitative analysis of the density of VEGFR-1 based upon flow cytometric analysis. Hypertrophy was induced by PE at a final concentration of $100 \mu \mathrm{M}$ for 48 hours in serum-free media. After PE treatment, cardiac myocytes were transfected with $10 \mathrm{nM}$ annealed siRNA targeting VEGFR-2. Lipofectamine 2000 was used as the transfecton reagent according to the manufacturer's instructions. After 48 hours of transfection, cells were trypsinized, collected, fixed and incubated with Alexa Fluor488 conjugated antibody of VEGFR-1 and Alexa Fluor 594 conjugated antibody to VEGFR-2. Cells were subjected to flow cytometry analysis and 30,000 cells in each sample were counted. Receptor density was determined by the intensity of fluorescence using the software FlowJo based on the data collection from 30,000 cells per sample. Data were plotted in two-dimensional fluorescence intensity vs. cell number. Each group of data presented were obtained from three independent experiments and each experiment contains a triplicate sample for each treatment. The values presented in the figures are means \pm SEM. $*$ Significantly different from Control group $(\mathrm{P}<0.05)$. MFI $=$ Median Fluorescence Intensity. $n$ $=3$. 


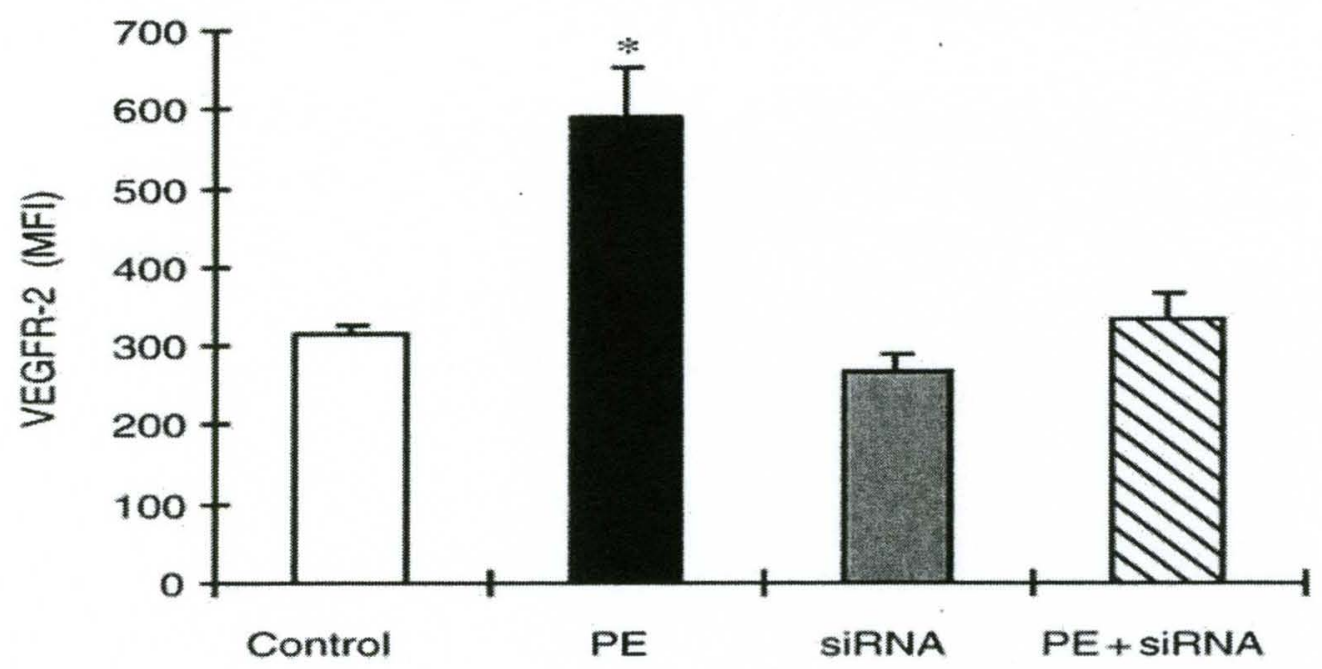

Figure 7. Quantitative analysis of the density of VEGFR-2 based upon flow cytometric analysis. Hypertrophy was induced by $\mathrm{PE}$ at a final concentration of $100 \mu \mathrm{M}$ for 48 hours in serum-free media. After PE treatment, cardiac myocytes were transfected with $10 \mathrm{nM}$ annealed siRNA targeting VEGFR-2. Lipofectamine 2000 was used as the transfecton reagent according to the manufacturer's instructions. After 48 hours of transfection, cells were trypsinized, collected, fixed and incubated with Alexa Fluor488 conjugated antibody of VEGFR-1 and Alexa Fluor 594 conjugated antibody to VEGFR-2. Cells were subjected to flow cytometry analysis and 30,000 cells in each sample were counted. Receptor density was determined by the intensity of fluorescence using the software FlowJo based on the data collection from 30,000 cells per sample. Data were plotted in two-dimensional fluorescence intensity vs. cell number. Each group of data presented were obtained from three independent experiments and each experiment contains a triplicate sample for each treatment. The values presented in the figures are means \pm SEM. $*$ Significantly different from Control group $(\mathrm{P}<0.05)$. MFI $=$ Median Fluorescence Intensity. $\mathrm{n}$ $=3$. 


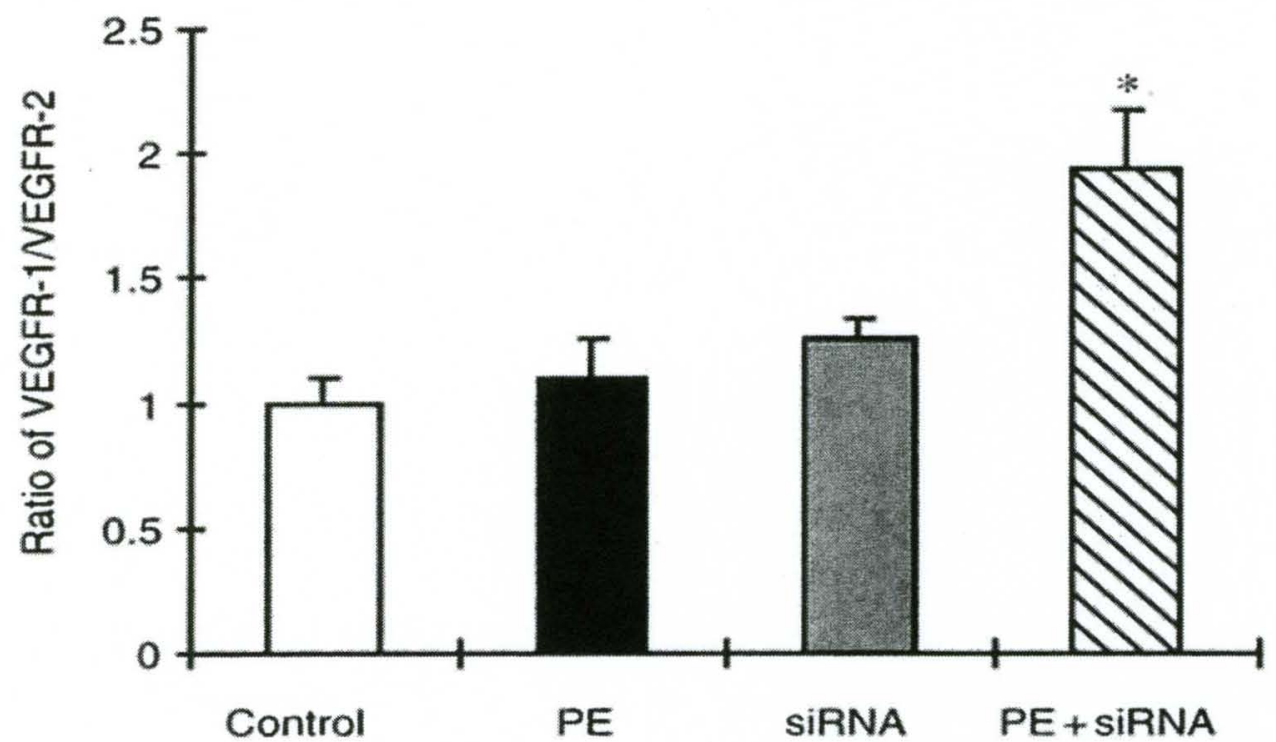

Figure 8. Ratio of VEGFR-1:VEGFR-2 after using siRNA targeting VEGFR-2. Hypertrophy was induced by $\mathrm{PE}$ at a final concentration of $100 \mu \mathrm{M}$ for 48 hours in serum-free media. After PE treatment, cardiac myocytes were transfected with $10 \mathrm{nM}$ annealed siRNA targeting VEGFR-2. Lipofectamine 2000 was used as the transfection reagent according to the manufacturer's instructions. After 48 hours of transfection, cells were trypsinized, collected, fixed and incubated with Alexa Fluor 488 conjugated antibody of VEGFR-1 and Alexa Fluor 594 conjugated antibody to VEGFR-2. Cells were subjected to flow cytometry analysis and 30,000 cells in each sample were counted. Receptor density was determined by the intensity of fluorescence using the software FlowJo based on the data collection from 30,000 cells per sample. Data were plotted in two-dimensional fluorescence intensity vs. cell number. Each group of data presented were obtained from three independent experiments and each experiment contains a triplicate sample for each treatment. The values presented in the figures are means \pm SEM. *Significantly different from Control group $(\mathrm{P}<0.05)$. MFI $=$ Median Fluorescence Intensity. $\mathrm{n}=3$. 


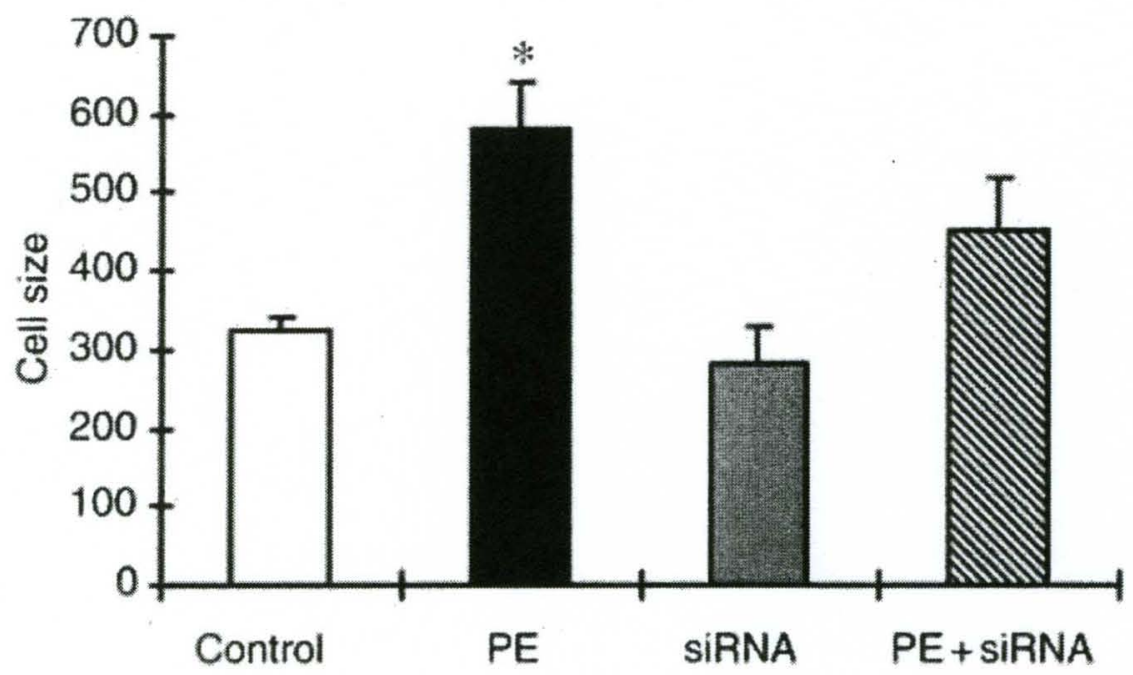

Figure 9. Regression of PE-induced cell hypertrophy by siRNA targeting VEGFR-2. Quantitative analysis of cell size based upon flow cytometric analysis. Hypertrophy was induced by PE at a final concentration of $100 \mu \mathrm{M}$ for 48 hours in serum-free media. After PE treatment, cardiac myocytes were transfected with $10 \mathrm{nM}$ annealed siRNA targeting VEGFR-2. Lipofectamine 2000 was used as the transfection reagent according to the manufacturer's instructions. After 48 hours of transfection, cells were trypsinized, collected, fixed and subjected to flow cytometry analysis using forward scatter light, which is proportional to the cell size. 30,000 cells in each sample were counted. Each group of data presented were obtained from three independent experiments and each experiment contains a triplicate sample for each treatment. The values presented in the figures are means \pm SEM. *Significantly different from Control group $(\mathrm{P}<0.05)$. $\mathrm{n}=3$. 


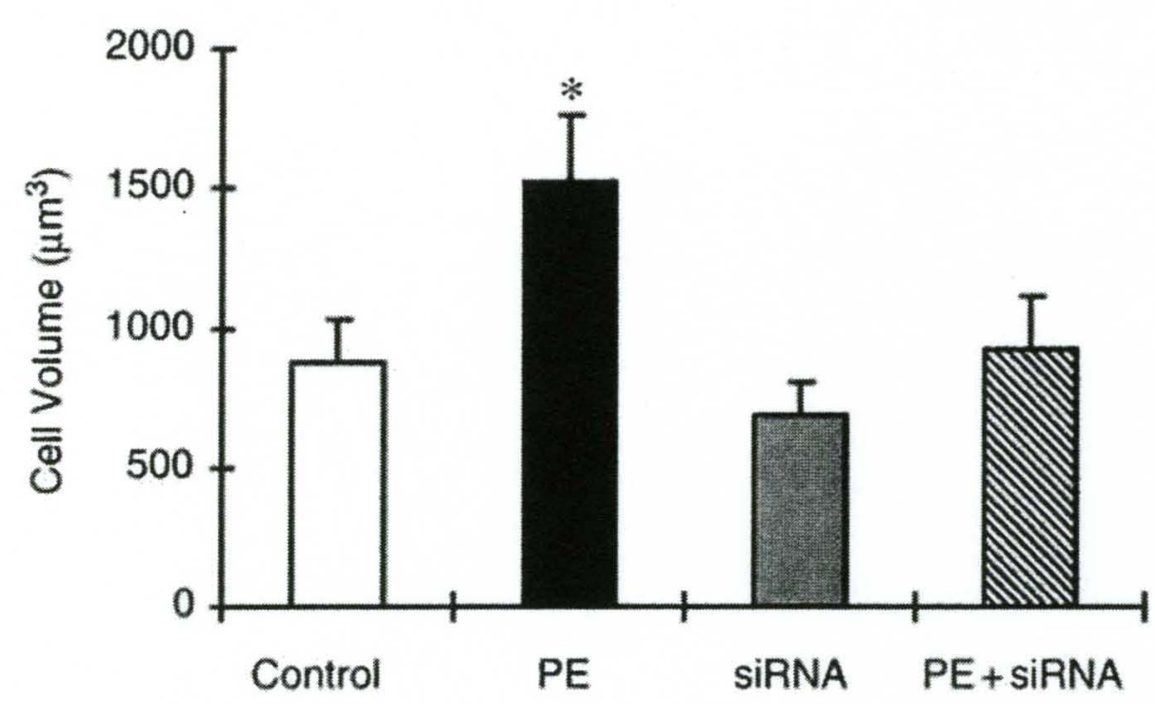

Figure 10. Regression of PE-induced cell hypertrophy by siRNA targeting VEGFR-2.

Hypertrophy was induced by $\mathrm{PE}$ at a final concentration of $100 \mu \mathrm{M}$ for 48 hours in serum-free media. After PE treatment, cardiac myocytes were transfected with $10 \mathrm{nM}$ annealed siRNA targeting VEGFR-2. Lipofectamine 2000 was used as the transfection reagent according to the manufacturer's instructions. After 48 hours of transfection, cells were trypsinized, collected. Cell volume was determined using a Lovins microslide field finder. Briefly, cardiac myocytes in cultures were trypsinized, suspended in PBS and loaded onto a microslide field finder. The diameters of approximately 150 cells from each group were assessed and recorded. The cell volume was calculated using the volume of a sphere: $V=(4 / 3) \pi r^{3}$ where $V=$ cell volume, $\pi=3.14$ and $\mathrm{r}=$ radius. Each group of data presented were obtained from three independent experiments and each experiment contains a triplicate sample for each treatment. The values presented in the figures are means \pm SEM. *Significantly different from Control group $(\mathrm{P}<0.05)$. $\mathrm{n}=3$. 


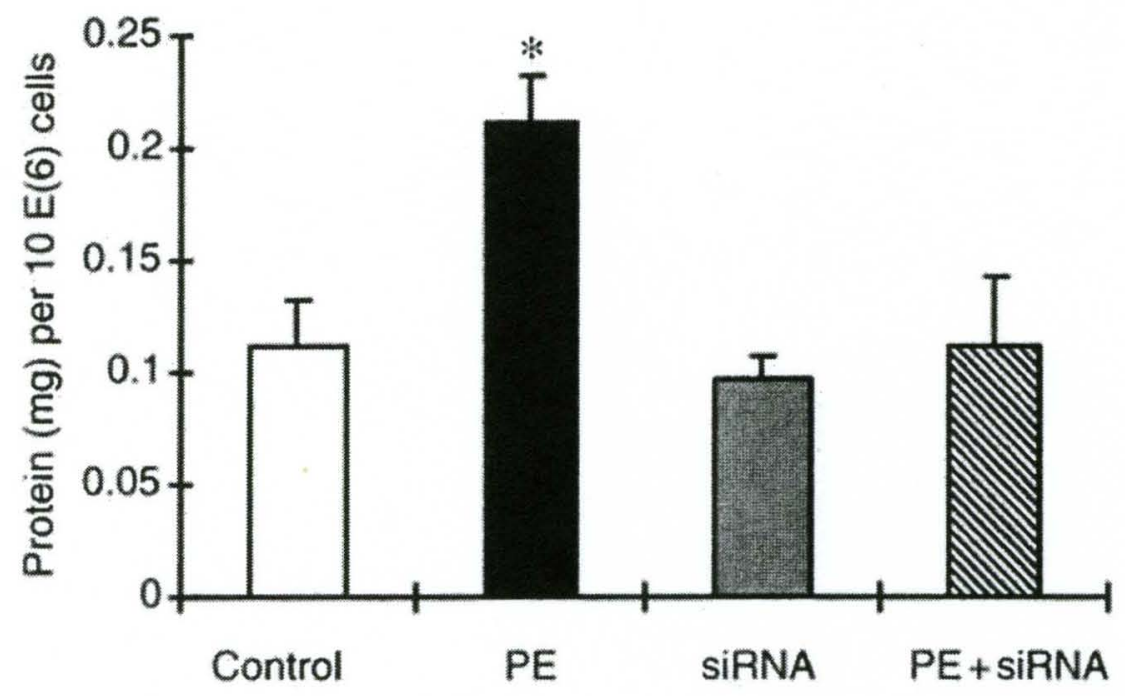

Figure 11. Regression of PE-induced cell hypertrophy by siRNA targeting VEGFR-2. The protein content in the cells measured by a Bradford method and normalized by cell number. Each group of data presented were obtained from three independent experiments and each experiment contains a triplicate sample for each treatment. The values presented in the figures are means \pm SEM.

*Significantly different from Control group $(\mathrm{P}<0.05)$. 


\section{Regression of PE-induced hypertrophy by siRNA targeting VEGFR-2 and the requirement of VEGF in the regression of cardiac myocyte hypertrophy.}

1. The regression of cardiac myocyte hypertrophy induced by $\mathrm{Cu}$ is blocked if a antiVEGF antibody is added, further demonstrating that VEGF is required for angiogenesis and growth, but also plays a role in the regression effect that we observe. Further, VEGFR-2 gene silencing-induced regression of cardiac myocyte hypertrophy is VEGF-dependent as well ${ }^{159}$. We have observed both in vivo and in vitro that the Cu-effect is VEGF-dependent. When silencing VEGFR-2, we observed the same phenomenon with $\mathrm{Cu}$. The next question we asked is: Is this effect also VEGF-dependent?

There are three indices of hypertrophy that we used to measure the effect of $\mathrm{Cu}$ on the reversal of hypertrophy: cell size, cell volume and protein content. Cell size was determined by flow cytometry, using forward scatter light, which is proportional to cell size. Cell volume was determined using a microslide field finder, and the diamter of 150 cells was measured. The equation for the volume of a sphere was used to determine the volume, $V=(4 / 3) \pi r^{3}$. Protein content was measured using the Bradford method, and was normalized by the cell number.

And as clearly shown in Figures 12, 13 and 14, the regression of PE-induced hypertrophy by siRNA targeting VEGFR-2 is indeed VEGF-dependent. 


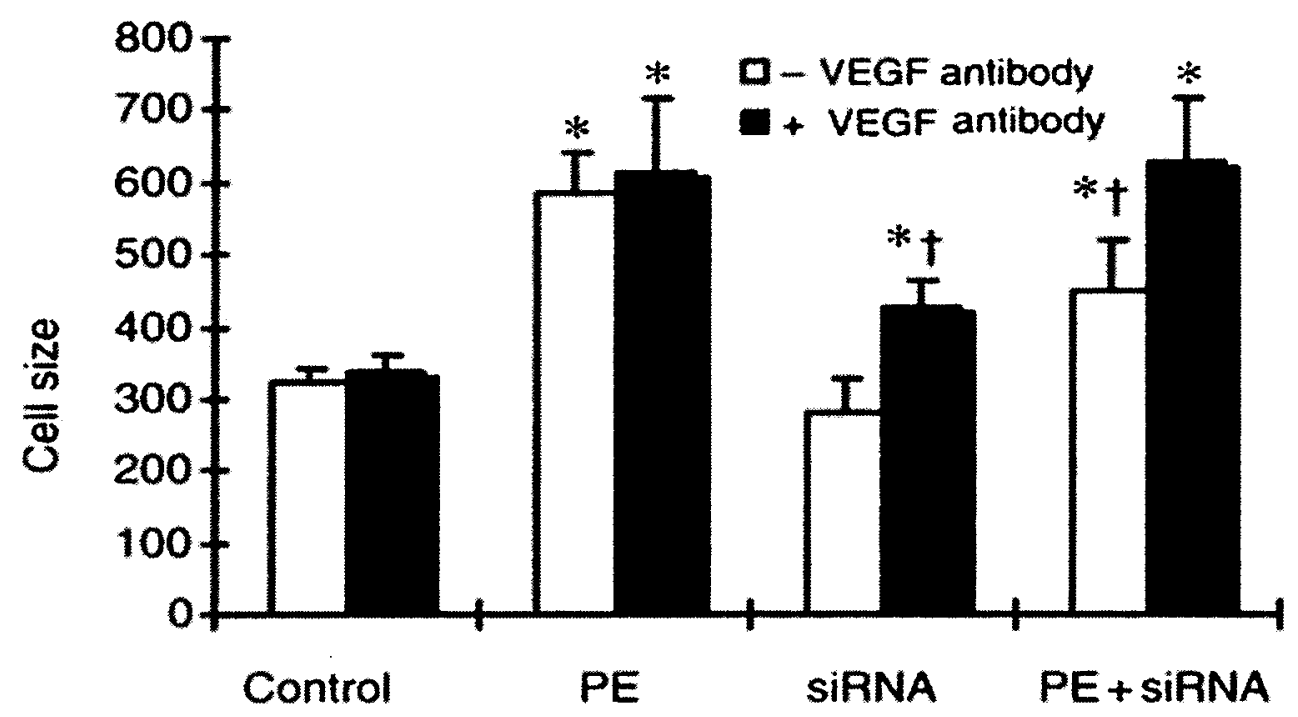

Figure 12. Regression of PE-induced hypertrophy by siRNA targeting VEGFR-2 and the requirement of VEGF in the regression of cardiac myocyte hypertrophy induced by siRNA targeting VEGFR-2. Quantitative analysis of cell size based upon flow cytometric analysis. Hypertrophy was induced by PE at a final concentration of $100 \mu \mathrm{M}$ for 48 hours in serum-free media. After PE treatment, cardiac myocytes were transfected with $10 \mathrm{nM}$ annealed siRNA targeting VEGFR-2. Lipofectamine 2000 was used as the transfection reagent according to the manufacturer's instructions. After $\mathbf{4 8}$ hours of transfection, cells were trypsinized, collected, fixed and subjected to flow cytometry analysis using forward scatter light, which is proportional to the cell size. 30,000 cells in each sample were counted. Each group of data presented were obtained from three independent experiments and each experiment contains a triplicate sample for each treatment. The values presented in the figures are means \pm SEM. *Significantly different from Control group. †significantly different from PE-treated group $(\mathrm{P}<0.05) . \mathrm{n}=3$. 


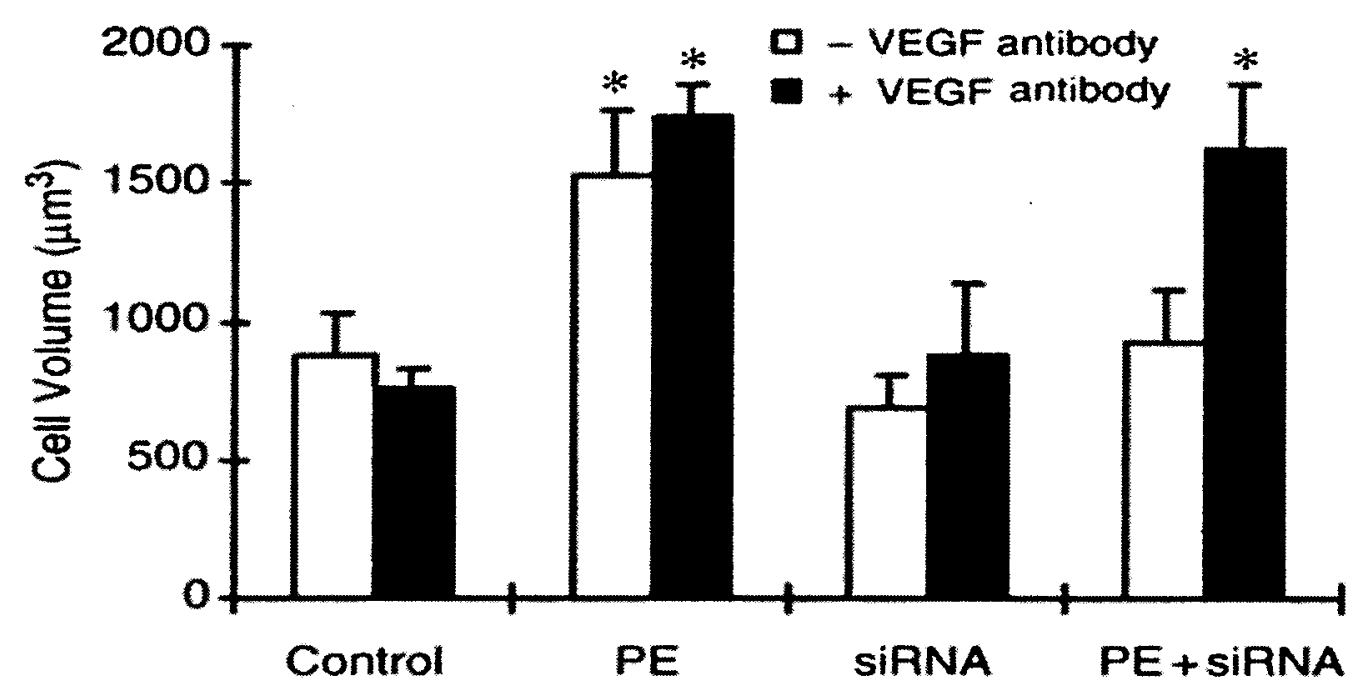

Figure 13. Effect of siRNA targeting VEGFR-2 on the regression of PE-induced hypertrophy, and the requirement of VEGF. Hypertrophy was induced by PE at a final concentration of $100 \mu \mathrm{M}$ for 48 hours in serum-free media. After PE treatment, cardiac myocytes were transfected with $10 \mathrm{nM}$ annealed siRNA targeting VEGFR-2. Lipofectamine 2000 was used as the transfection reagent according to the manufacturer's instructions. After 48 hours of transfection, cells were trypsinized, collected. Cell volume was determined using a Lovins microslide field finder. Briefly, cardiac myocytes in cultures were trypsinized, suspended in PBS and loaded onto a microslide field finder. The diameters of approximately 150 cells from each group were assessed and recorded. The cell volume was calculated using the volume of a sphere: $V=(4 / 3) \pi r^{3}$ where $V$ $=$ cell volume, $\pi=3.14$ and $r=$ radius. Each group of data presented were obtained from three independent experiments and each experiment contains a triplicate sample for each treatment. The values presented in the figures are means \pm SEM. ${ }^{*}$ Significantly different from Control group $(\mathrm{P}<$ $0.05) . \mathrm{n}=3$. 


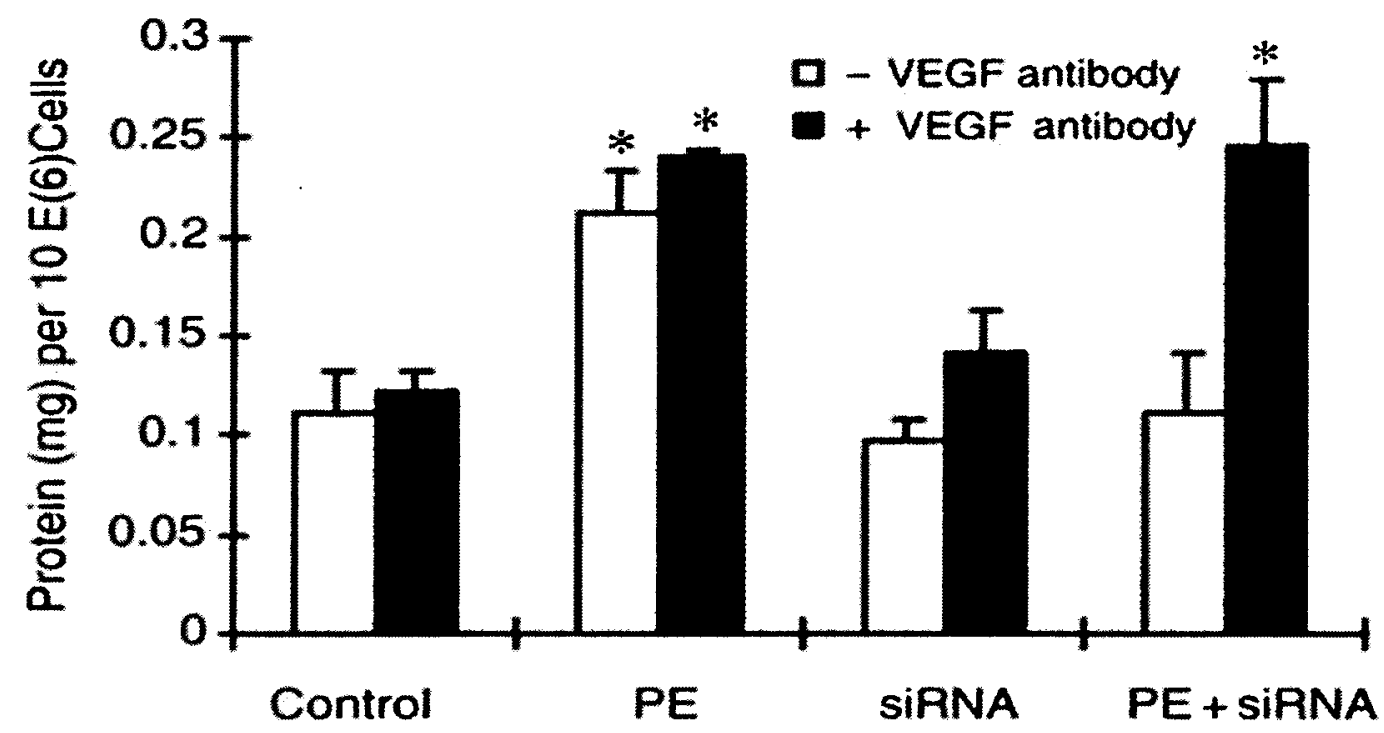

Figure 14. Regression of PE-induced cell hypertrophy by siRNA targeting VEGFR-2in primary cultures of neonatal rat cardiomyocytes, and the requirement of VEGF in the regression of cardiomyocyte hypertrophy induced by siRNA targeting VEGFR-2. The protein content in the cells measured by a Bradford method and normalized by cell number. Each group of data presented were obtained from three independent experiments and each experiment contains a triplicate sample for each treatment. The values presented in the figures are means \pm SEM. *Significantly different from Control group $(\mathrm{P}<0.05)$. 
E. Suppression of $\mathrm{Cu}$-induced reversal of cardiomyocyte hypertrophy by gene silencing of VEGFR-1.

1. Up until this point, all the data points to VEGFR-1. The increase in the ratio of VEGFR-1/VEGFR-2 accompanied with the reversal of PE-induced cardiac myocyte hypertrophy indicated the determinant role of VEGFR-1 in $\mathrm{Cu}$ regression of cardiac myocyte hypertrophy. The next question that we asked was: If you knock out VEGFR-1, what happens? We used siRNA targeting VEGFR-1 to examine the effect of suppressing VEGFR-1 on $\mathrm{Cu}$ reversal of cardiac myocyte hypertrophy. Figures 15, 16 and 17 show quantitative analysis of the effect of siRNA targeting VEGFR-1 on VEGFR-1, VEGFR-2 and the ratio of VEGFR1:VEGFR-2 respectively. As shown in Figures 18, 19 and 20, gene silencing of VEGFR-1 inhibited the response of the cells to PE-induced VEGFR-1 elevation, and consequently suppressed the increase in the ratio of VEGFR-1/VEGFR-2. 


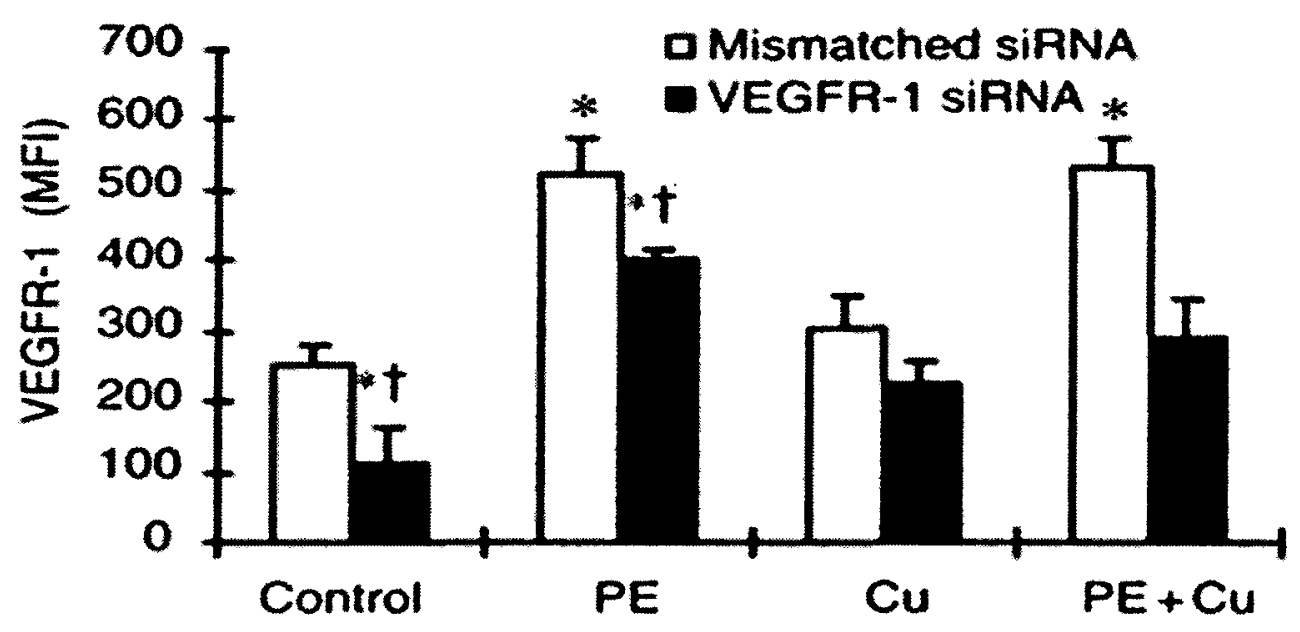

Figure 15. Effect of siRNA targeting VEGFR-1 on the distribution of VEGFR-1 and VEGFR-2 and the antagonistic effect of siRNA targeting VEGFR-1 on Cu-induced regression of cell hypertrophy. Quantitative analysis of the density of VEGFR-1 based on the flow cytometric analysis. Hypertrophy was induced by PE at a final concentration of $100 \mu \mathrm{M}$ for 48 hours in serum-free media. After PE treatment, cardiac myocytes were transfected with $10 \mathrm{nM}$ annealed siRNA targeting VEGFR-1. Lipofectamine 2000 was used as the transfection reagent according to the manufacturer's instructions. After 48 hours of transfection, cells were trypsinized, collected, fixed and incubated with Alexa Fluor488 conjugated antibody of VEGFR-1 and Alexa Fluor 594 conjugated antibody to VEGFR-2. Cells were subjected to flow cytometry analysis and 30,000 cells in each sample were counted. Receptor density was determined by the intensity of fluorescence using the software FlowJo based on the data collection from 30,000 cells per sample. Data were plotted in two-dimensional fluorescence intensity vs. cell number. Each group of data presented were obtained from three independent experiments and each experiment contains a triplicate sample for each treatment. The values presented in the figures are means \pm SEM.

*Significantly different from Control group. †significantly different from $\mathrm{PE}$-treated group $(\mathrm{P}<$ 0.05 ). $\mathrm{MFI}=$ Median Fluorescence Intensity. $\mathrm{n}=3$. 


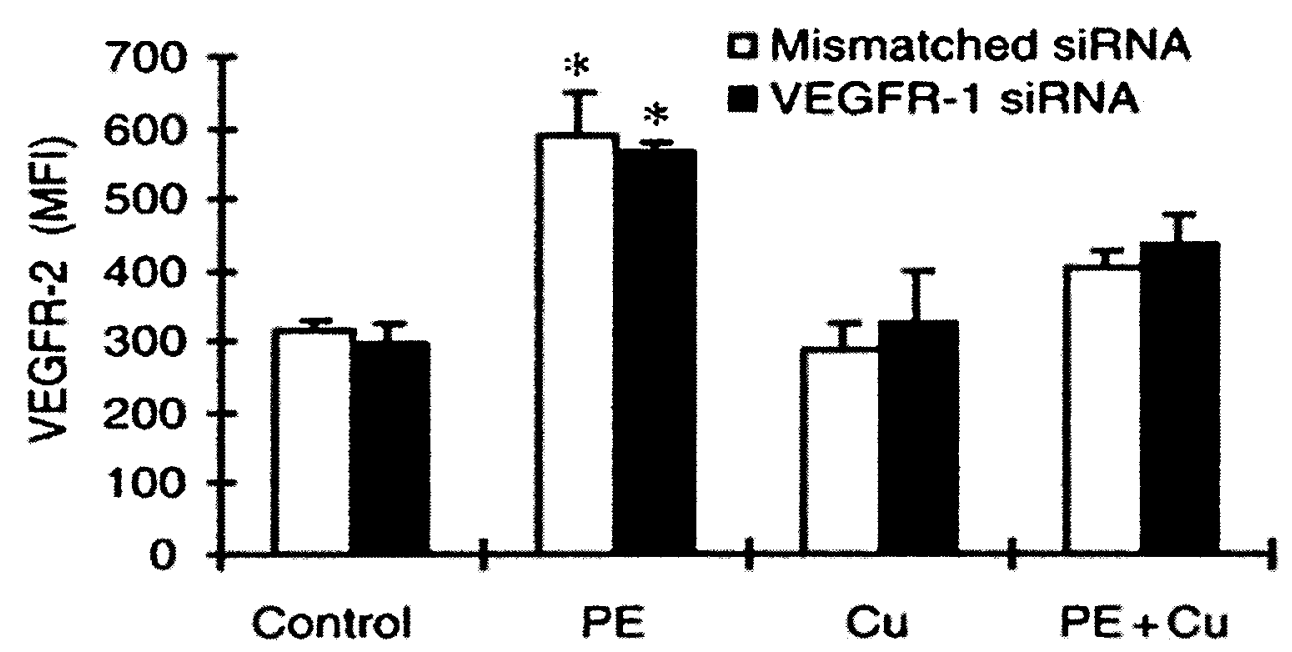

Figure 16. Effect of siRNA targeting VEGFR-1 on the distribution of VEGFR-1 and VEGFR-2 and the antagonistic effect of siRNA targeting VEGFR-1 on $\mathrm{Cu}$-induced regression of cell hypertrophy. Quantitative analysis of the density of VEGFR-1 based on the flow cytometric analysis. Hypertrophy was induced by PE at a final concentration of $100 \mu \mathrm{M}$ for 48 hours in serum-free media. After PE treatment, cardiac myocytes were transfected with $10 \mathrm{nM}$ annealed siRNA targeting VEGFR-1. Lipofectamine 2000 was used as the transfection reagent according to the manufacturer's instructions. After 48 hours of transfection, cells were trypsinized, collected, fixed and incubated with Alexa Fluor 488 conjugated antibody of VEGFR-1 and Alexa Fluor 594 conjugated antibody to VEGFR-2. Cells were subjected to flow cytometry analysis and 30,000 cells in each sample were counted. Receptor density was determined by the intensity of fluorescence using the software FlowJo based on the data collection from 30,000 cells per sample. Data were plotted in two-dimensional fluorescence intensity vs. cell number. Each group of data presented were obtained from three independent experiments and each experiment contains a triplicate sample for each treatment. The values presented in the figures are means \pm SEM. *Significantly different from Control group $(\mathrm{P}<0.05)$. MFI = Median Fluorescence Intensity. $\mathrm{n}$ $=3$. 


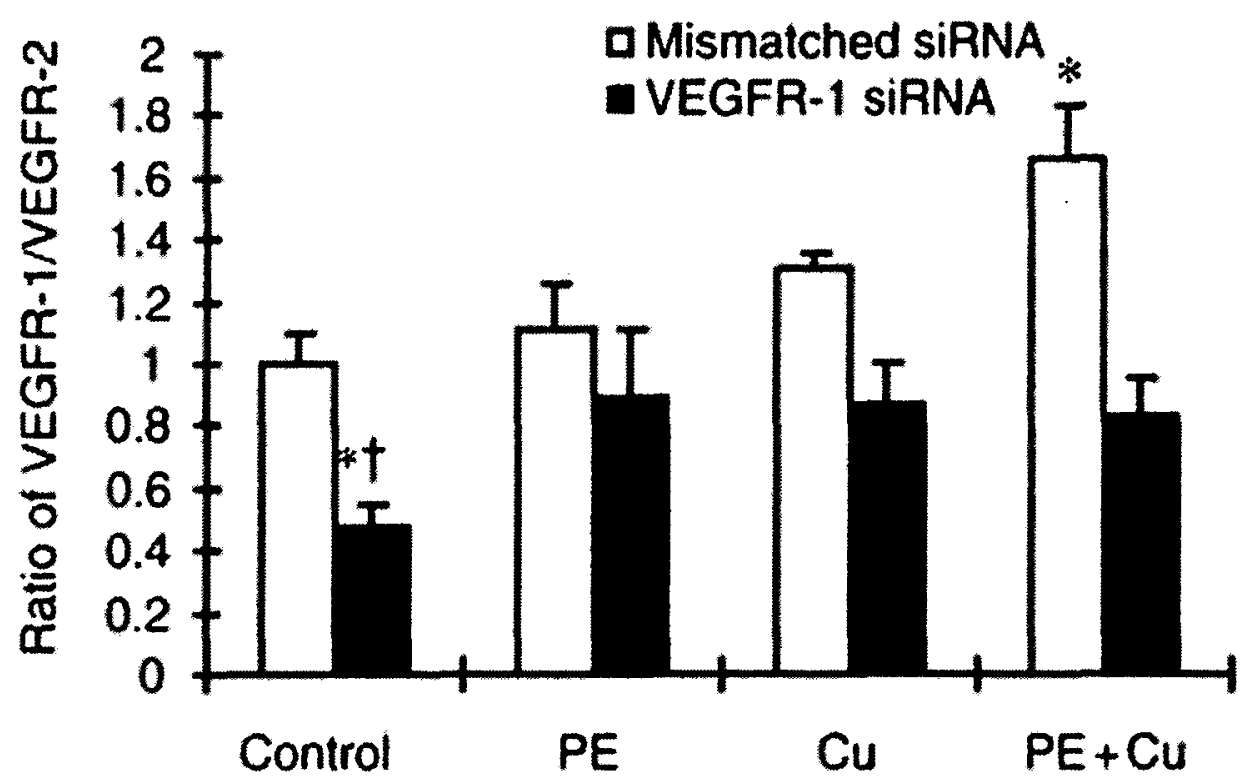

Figure 17. Quantitative analysis of the density of VEGFR-1 based on the flow cytometric analysis. Ratio of VEGFR-1:VEGFR-2. Effect of siRNA targeting VEGFR-1 on the distribution of VEGFR-1 and VEGFR-2 and the antagonistic effect of siRNA targeting VEGFR-1 on Cu-induced regression of cell hypertrophy. Effect of siRNA targeting VEGFR-1 on the distribution of VEGFR-1 and VEGFR-2 and the antagonistic effect of siRNA targeting VEGFR-1 on Cu-induced regression of cell hypertrophy. Hypertrophy was induced by $P E$ at a final concentration of 100 $\mu \mathrm{M}$ for 48 hours in serum-free media. After PE treatment, cardiac myocytes were transfected with $10 \mathrm{nM}$ annealed siRNA targeting VEGFR-1. Lipofectamine 2000 was used as the transfection reagent according to the manufacturer's instructions. After 48 hours of transfection, cells were trypsinized, collected, fixed and incubated with Alexa Fluor 488 conjugated antibody of VEGFR-1 and Alexa Fluor 594 conjugated antibody to VEGFR-2. Cells were subjected to flow cytometry analysis and 30,000 cells in each sample were counted. Receptor density was determined by the intensity of fluorescence using the software FlowJo based on the data collection from 30,000 cells per sample. Data were plotted in two-dimensional fluorescence intensity vs. cell number. Each group of data presented were obtained from three independent experiments and each experiment contains a triplicate sample for each treatment. The values presented in the figures are means \pm SEM. * Significantly different from Control group. $\dagger$ significantly different from PE-treated group $(\mathrm{P}<0.05)$. MFI $=$ Median Fluorescence Intensity. $\mathrm{n}=3$. 
Again, we measured the three indices of hypertrophy: cell size, cell volume and protein content. Cell size was determined by flow cytometry, using forward scatter light, which is proportional to cell size. Cell volume was determined using a microslide field finder, and the diamter of 150 cells was measured. The equation for the volume of a sphere was used to determine the volume, $V=(4 / 3) \pi r^{3}$. Protein content was measured using the Bradford method, and was normalized by the cell number.

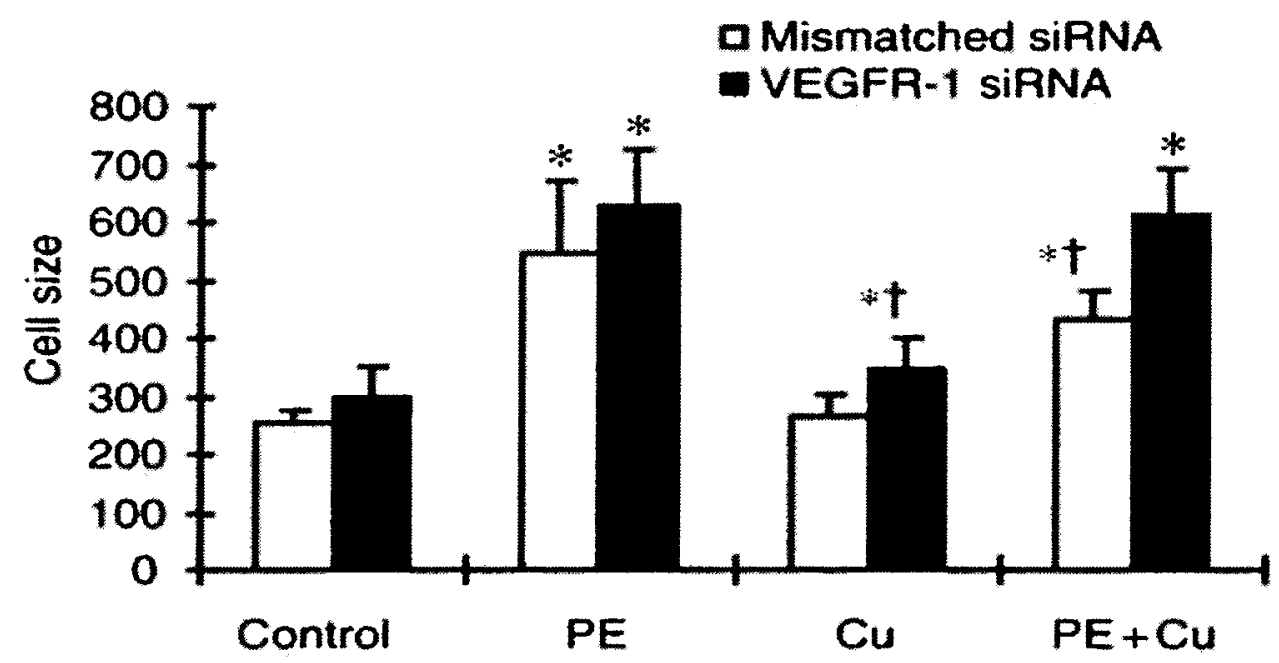

Figure 18. Quantitative analysis of cell size based upon flow cytometric analysis. Hypertrophy was induced by $\mathrm{PE}$ at a final concentration of $100 \mu \mathrm{M}$ for 48 hours in serum-free media. After PE treatment, cardiac myocytes were transfected with $10 \mathrm{nM}$ annealed siRNA targeting VEGFR-1. Lipofectamine 2000 was used as the transfection reagent according to the manufacturer's instructions. After 48 hours of transfection, cells were trypsinized, collected, fixed and subjected to flow cytometry analysis using forward scatter light, which is proportional to the cell size. 30,000 cells in each sample were counted. Each group of data presented were obtained from three independent experiments and each experiment contains a triplicate sample for each treatment. The values presented in the figures are means \pm SEM. *Significantly different from Control group. † significantly different from $P E-$ treated group $(\mathrm{P}<0.05) . \mathrm{n}=3$. 


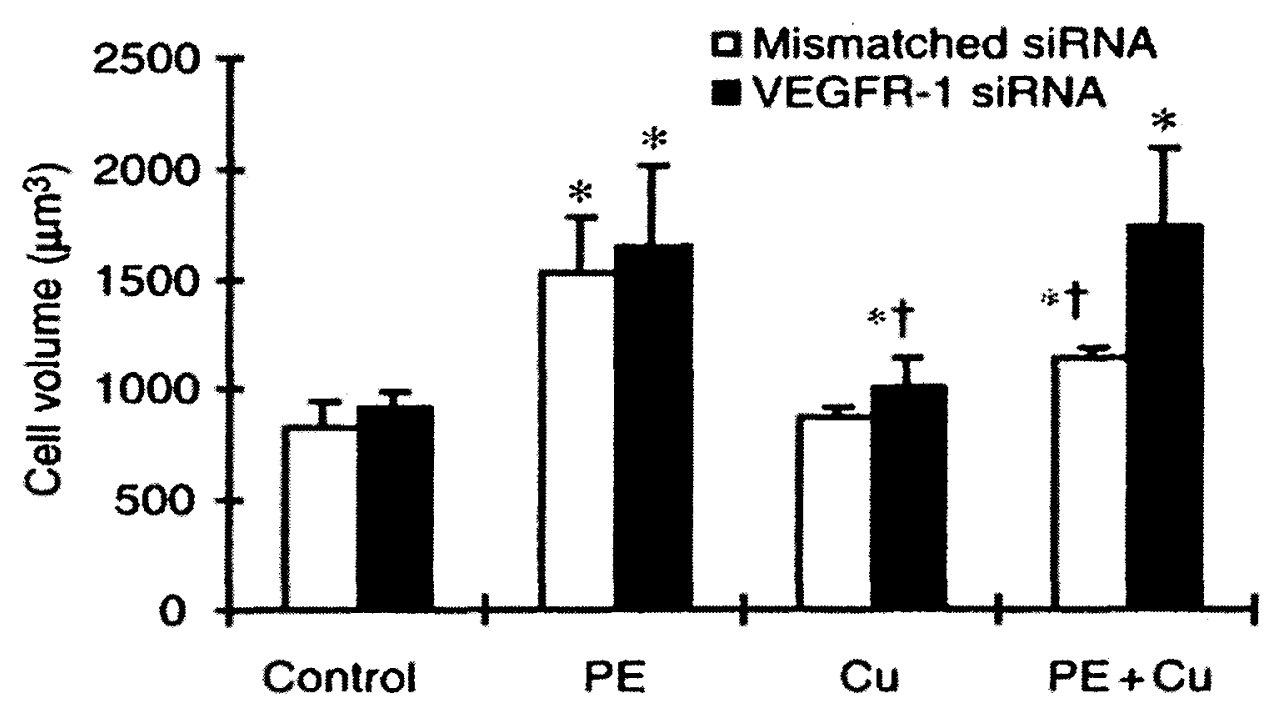

Figure 19. Hypertrophy was induced by PE at a final concentration of $100 \mu \mathrm{M}$ for 48 hours in serum-free media. After PE treatment, cardiac myocytes were transfected with $10 \mathrm{nM}$ annealed siRNA targeting VEGFR-1. Lipofectamine 2000 was used as the transfection reagent according to the manufacturer's instructions. After 48 hours of transfection, cells were trypsinized and collected. Cell volume was determined using a Lovins microslide field finder. Briefly, cardiac myocytes in cultures were trypsinized, suspended in PBS and loaded onto a microslide field finder. The diameters of approximately 150 cells from each group were assessed and recorded. The cell volume was calculatred using the volume of a sphere: $V=(4 / 3) \pi r^{3}$ where $V=$ cell volume, $\pi=3.14$ and $r=$ radius. Each group of data presented were obtained from three independent experiments and each experiment contains a triplicate sample for each treatment. The values presented in the figures are means \pm SEM. *Significantly different from Control group and $\dagger$ significantly different from $\mathrm{PE}$-treated group $(\mathrm{P}<0.05)$. 


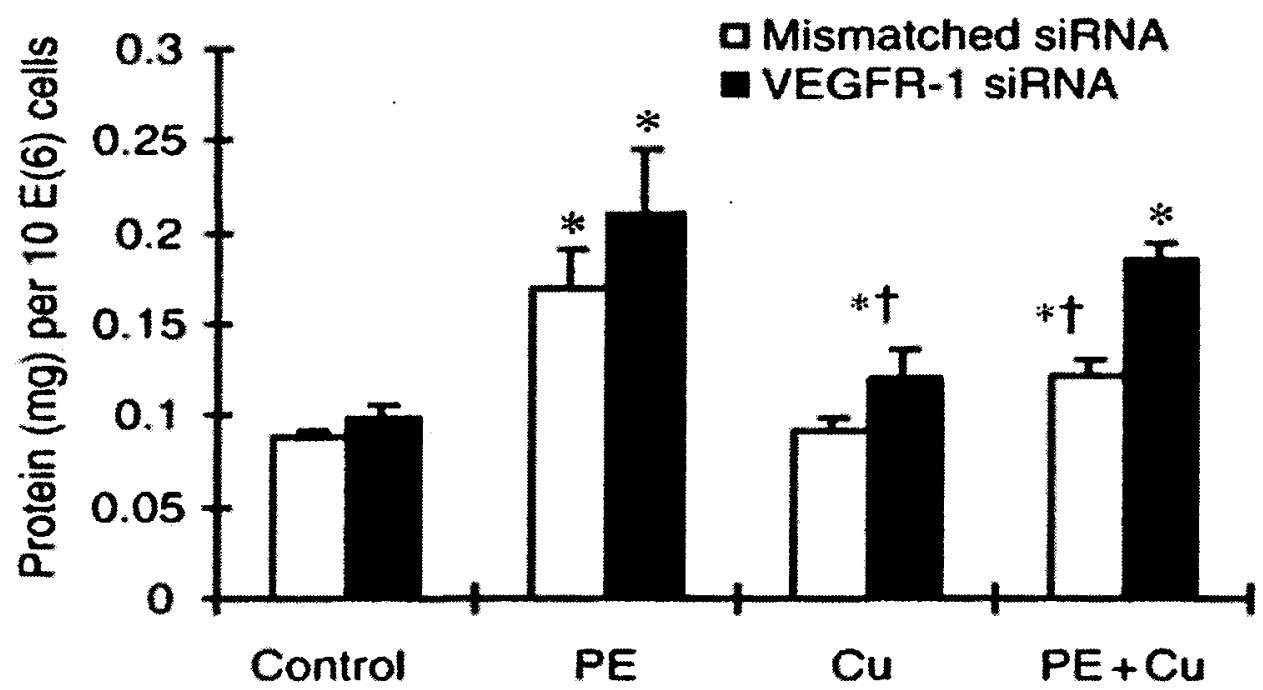

Figure 20. Effect of siRNA targeting VEGFR-1 on the distribution of VEGFR-1 and VEGFR-2 and the antagonistic effect of siRNA targeting VEGFR- 1 on Cu-induced regression of cell hypertrophy. At the end of siRNA treatment, cells were collected. The protein content in the cells measured by a Bradford method and normalized by cell number. Each group of data presented were obtained from three independent experiments and each experiment contains a triplicate sample for each treatment. The values presented in the figures are means \pm SEM. *Significantly different from Control group and $\dagger$ significantly different from PE-treated group $(\mathrm{P}<0.05)$.

In summary, the data suggests that the regression of hypertrophy is associated with an increase in the VEGFR-1:VEGFR-2. We also observed that siRNA targeting VEGFR-2 mimics the Cu-effect. Additionally, we also observed that siRNA targeting VEGFR-1 blocks Cu-induced reversal of cardiac myocyte hypertrophy induced by PE. 


\section{F. Association of PKG-1 with VEGFR-1-mediated reversal of cardiomyocyte hypertrophy by $\mathrm{Cu}$ treatment}

1. The data thus far suggests that VEGFR-1 is a mediator in the reversal effect. The next question that we asked was: What is the signaling molecule associated with VEGFR-1? cGMP-dependent protein kinase-1 (PKG-1) has been defined to be involved in the inhibition of myocardial growth. The PKG-1 signaling pathway is a well-studied and important pathway that has also been shown to be involved in the regression of cardiac hypertrophy ${ }^{176}$. Additionally, this effect has also been shown to be VEGF-dependent. The link between VEGFR-1 and PKG-1 was examined by the effect of VEGFR-1 gene silencing on PKG-1 activity.

We hypothesized that the VEGFR-1 increase related regression is mediated by PKG-1 and set out to determine the role of PKG-1 and VEGFR-1 mediated regression of cardiac myocyte hypertrophy. The first thing that we examined was the effect of VEGFR-1 gene silencing on PKG-1 activity, as seen in Figure 21. 


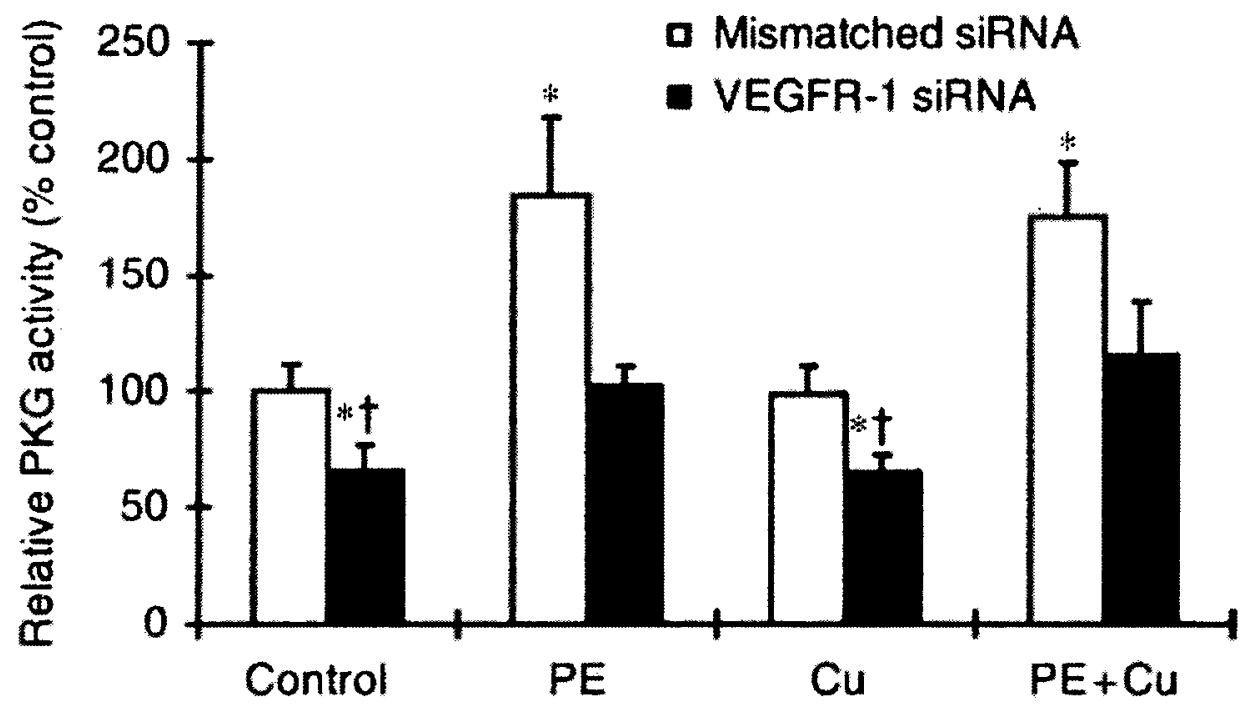

Figure 21. Inhibitory effect of siRNA targeting VEGFR-1 on PKG-1 activity on PE-elevated PKG-1 activity. PKG-1 activity was assayed by a colormetric analysis procedure. The assay was performed 30 minutes after treatment with $\mathrm{Cu}$. Each group of data presented were obtained from three independent experiments and each experiment contains a triplicate sample for each treatment. The values presented in the figures are means \pm SEM. *Significantly different from Control group and $\dagger$ significantly different from $\mathrm{PE}$-treated group $(\mathrm{P}<0.05)$. 
2. We also examined the effect of PKG-1 antagonist on $\mathrm{Cu}$ - or VEGFR-2 gene silencing-induced regression of cardiac myocyte hypertrophy. The results suggest that PE treatment significantly elevated PKG-1 activity and $\mathrm{Cu}$ addition did not change PKG-1 activity, but VEGFR-1 gene silencing significantly attenuated PEinduced PKG-1 activation. Additionally, a PKG-1 antagonist, Rp-8-pCPTcGMPS, blocked both VEGFR-2 gene silencing- and $\mathrm{Cu}$-induced reversal of cardiac myocyte hypertrophy, as shown in Figures 22-24 and 25-27 respectively .

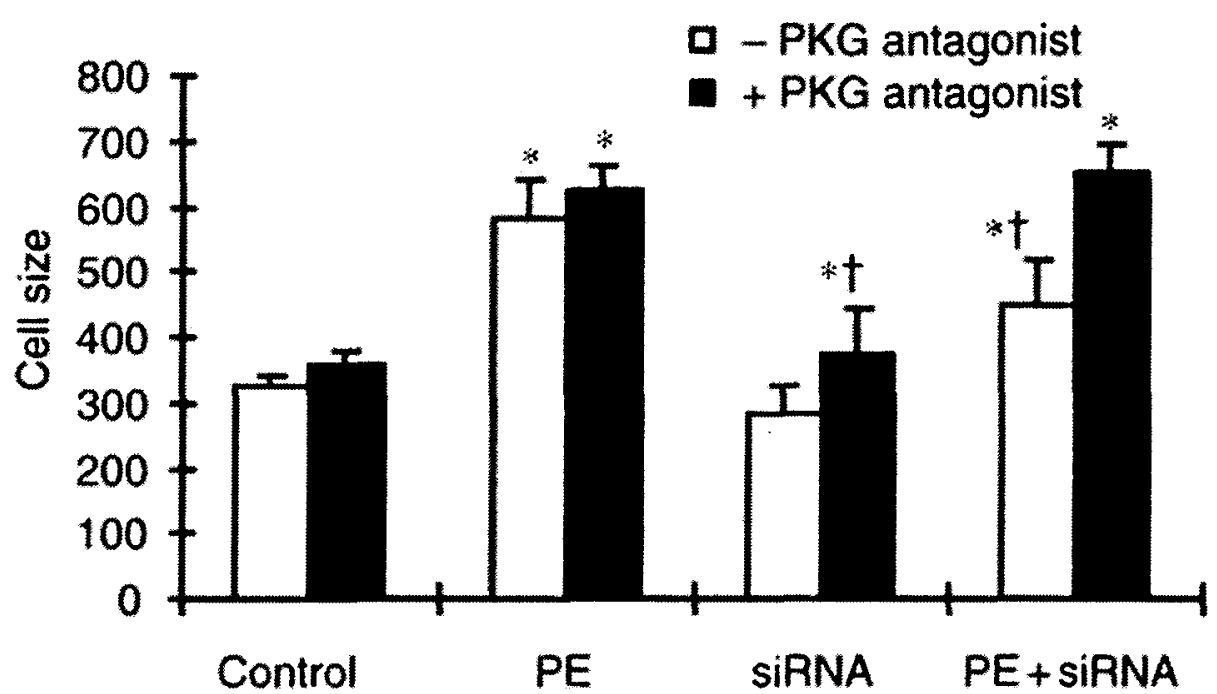

Figure 22. Antagonistic effect PKG-1 inhibitor, Rp-8-pCPT-cGMPS, on regression of cell hypertrophy by siRNA targeting VEGFR-2. PKG- 1 inhibitor was added 1 hour prior to siRNA at a concentration of $0.5 \mu \mathrm{M}$. Hypertrophy was induced by $\mathrm{PE}$ at a final concentration of $100 \mu \mathrm{M}$ for 48 hours in serum-free media. After PE treatment, cardiac myocytes were transfected with $10 \mathrm{nM}$ annealed siRNA targeting VEGFR-2. Lipofectamine 2000 was used as the transfection reagent according to the manufacturer's instructions. After 48 hours of transfection, cells were trypsinized and collected. Quantitative analysis of cell size based upon flow cytometric analysis. Each group of data presented were obtained from three independent experiments and each experiment contains a triplicate sample for each treatment. The values presented in the figures are means \pm SEM. *Significantly different from Control group and $\nmid$ significantly different from PE-treated group (P $<0.05)$. 


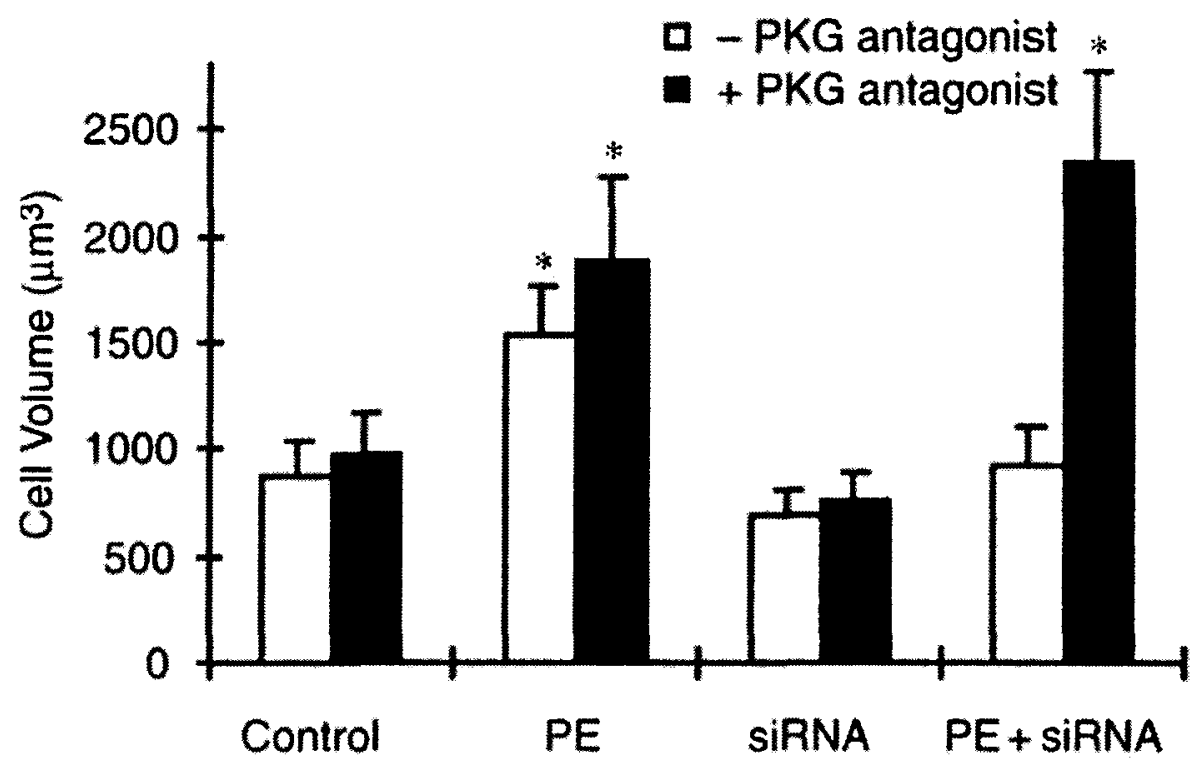

Figure 23. Antagonistic effect PKG-1 inhibitor, Rp-8-pCPT-cGMPS, on regression of cell hypertrophy by siRNA targeting VEGFR-2. PKG-1 inhibitor was added 1 hour prior to siRNA at a concentration of $0.5 \mu \mathrm{M}$. Hypertrophy was induced by PE at a final concentration of $100 \mu \mathrm{M}$ for 48 hours in serum-free media. After PE treatment, cardiac myocytes were transfected with $10 \mathrm{nM}$ annealed siRNA targeting VEGFR-2. Lipofectamine 2000 was used as the transfection reagent according to the manufacturer's instructions. After 48 hours of transfection, cells were trypsinized and collected. Cell volume was determined using a Lovins microslide field finder. Briefly, cardiac myocytes in cultures were trypsinized, suspended in PBS and loaded onto a microslide field finder. The diameters of approximately 150 cells from each group were assessed and recorded. The cell volume was calculated using the volume of a sphere: $V=(4 / 3) \pi r^{3}$ where $V=$ cell volume, $\pi=3.14$ and $r=$ radius. Each group of data presented were obtained from three independent experiments and each experiment contains a triplicate sample for each treatment. The values presented in the figures are means \pm SEM. *Significantly different from Control group $(\mathbf{P}<$ $0.05)$. 


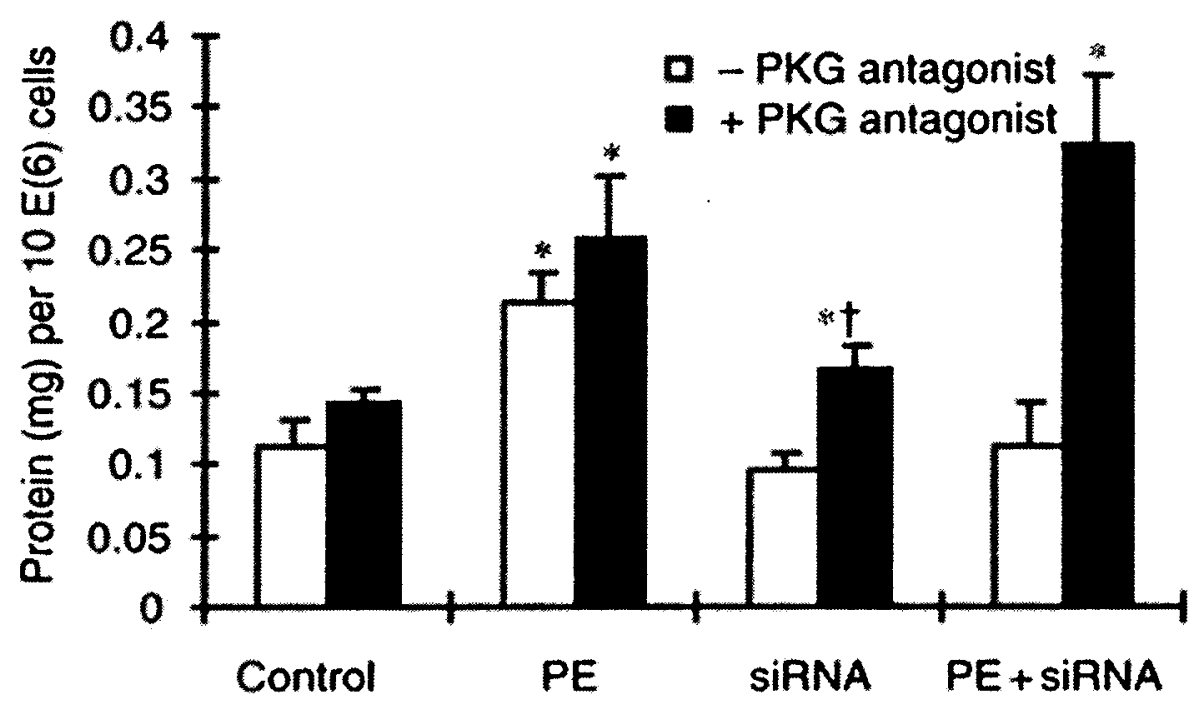

Figure 24. Antagonistic effect of PKG-1 inhibitor, Rp-8-pCPT-cGMPS, on regression of cell hypertrophy induced by siRNA targeting VEGFR-2. PKG-1 inhibitor was added 1 hour prior to siRNA at a concentration of $0.5 \mu \mathrm{M}$. Hypertrophy was induced by $\mathrm{PE}$ at a final concentration of $100 \mu \mathrm{M}$ for 48 hours in serum-free media. After PE treatment, cardiac myocytes were transfected with $10 \mathrm{nM}$ annealed siRNA targeting VEGFR-2. Lipofectamine 2000 was used as the transfection reagent according to the manufacturer's instructions. After 48 hours of transfection, cells were trypsinized and collected. The protein content in the cells measured by a Bradford method and normalized by cell number. Each group of data presented were obtained from three independent experiments and each experiment contains a triplicate sample for each treatment. The values presented in the figures are means \pm SEM. $*$ Significantly different from Control group and †significantly different from PE-treated group $(\mathrm{P}<0.05)$. 


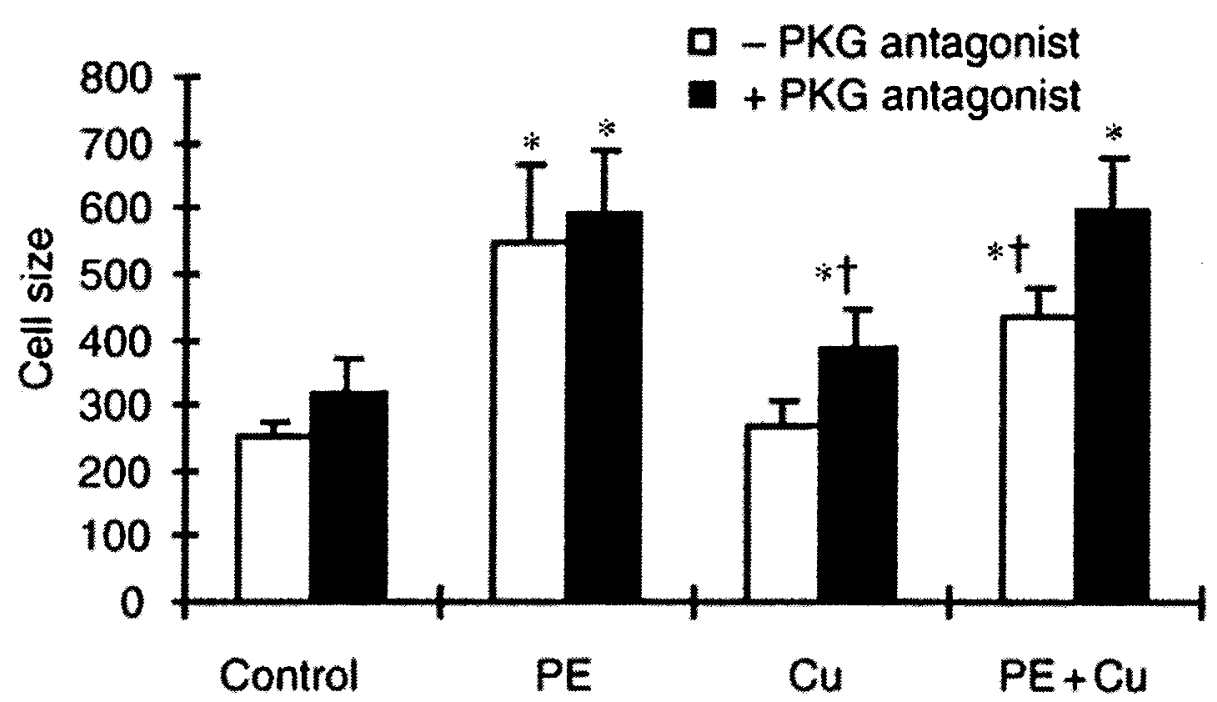

Figure 25. Antagonistic effect PKG-1 inhibitor, Rp-8-pCPT-cGMPS, on regression of cell hypertrophy by $\mathrm{Cu}$ supplementation. PKG-1 inhibitor was added $\mathrm{I}$ hour prior to $\mathrm{Cu}$ at a concentration of $0.5 \mu \mathrm{M}$. Hypertrophy was induced by $\mathrm{PE}$ at a final concentration of $100 \mu \mathrm{M}$ for 48 hours in serum-free media. After PE treatment, cardiac myocytes were treated with $5 \mu \mathrm{M} \mathrm{Cu}$ by direct addition to the cultures. Quantitative analysis of cell size based upon flow cytometric analysis. Each group of data presented were obtained from three independent experiments and each experiment contains a triplicate sample for each treatment. The values presented in the figures are means \pm SEM. *Significantly different from Control group and †significantly different from PE-treated group $(\mathrm{P}<0.05)$. 


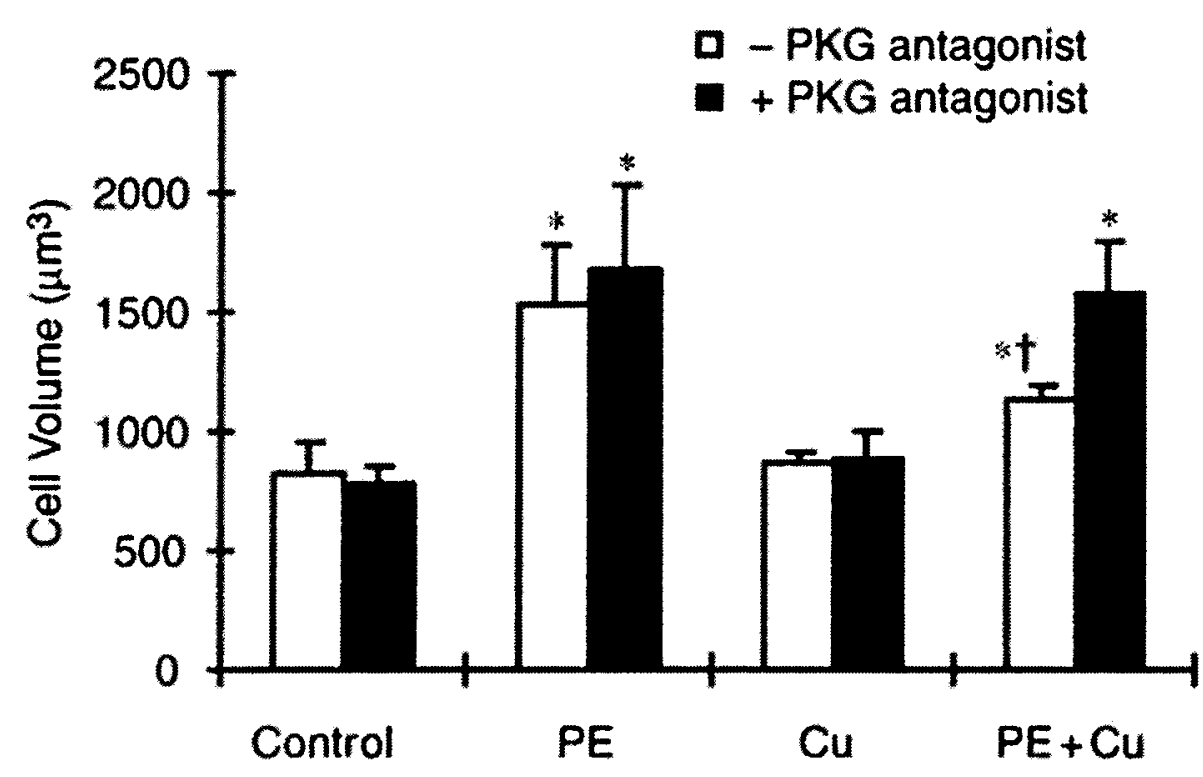

Figure 26. Antagonistic effect PKG-1 inhibitor, Rp-8-pCPT-cGMPS, on regression of cell hypertrophy by $\mathrm{Cu}$ supplementation. PKG-1 inhibitor was added 1 hour prior to $\mathrm{Cu}$ at a concentration of $0.5 \mu \mathrm{M}$. Hypertrophy was induced by PE at a final concentration of $100 \mu \mathrm{M}$ for 48 hours in serum-free media. After PE treatment, cardiac myocytes were treated with $5 \mu \mathrm{M} \mathrm{Cu}$ by direct addition to the cultures. Cell volume was determined using a Lovins microslide field finder. Briefly, cardiac myocytes in cultures were trypsinized, suspended in PBS and loaded onto a microslide field finder. The diameters of approximately 150 cells from each group were assessed and recorded. The cell volume was calculatred using the volume of a sphere: $V=(4 / 3) \pi r^{3}$ where $V=$ cell volume, $\pi=3.14$ and $r=$ radius. Each group of data presented were obtained from three independent experiments and each experiment contains a triplicate sample for each treatment. The values presented in the figures are means \pm SEM. *Significantly different from Control group and $\uparrow$ significantly different from PE-treated group $(\mathrm{P}<$ 0.05 ). 


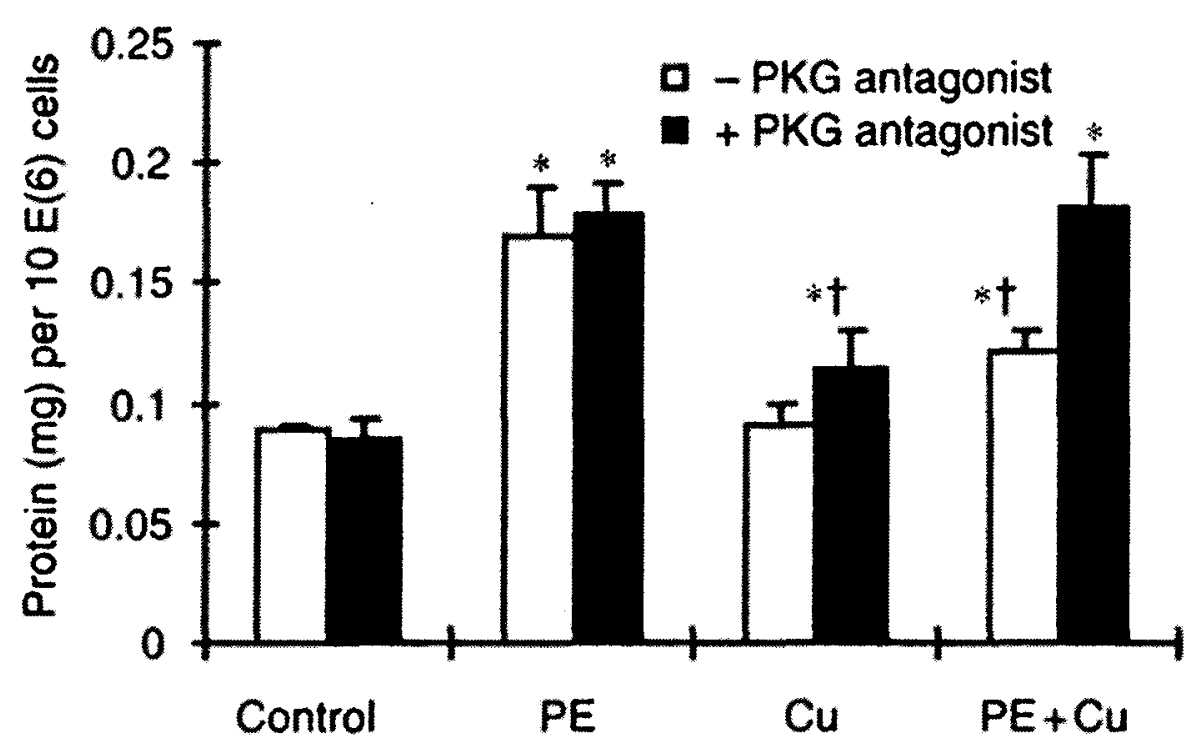

Figure 27. Antagonistic effect PKG-1 inhibitor, Rp-8-pCPT-cGMPS, on regression of cell hypertrophy by $\mathrm{Cu}$ supplementation. PKG-1 inhibitor was added 1 hour prior to $\mathrm{Cu}$ at a concentration of $0.5 \mu \mathrm{M}$. Hypertrophy was induced by $\mathrm{PE}$ at a final concentration of $100 \mu \mathrm{M}$ for 48 hours in serum-free media. After PE treatment, cardiac myocytes were treated with $5 \mu \mathrm{M} \mathrm{Cu}$ by direct addition to the cultures. The protein content in the cells measured by a Bradford method and normalized by cell number. Each group of data presented were obtained from three independent experiments and each experiment contains a triplicate sample for each treatment. The values presented in the figures are means \pm SEM. ${ }^{*}$ Significantly different from Control group and $\dagger$ significantly different from PE-treated group $(\mathrm{P}<0.05)$. 
In summary, these data suggest that PKG-1 antagonist blocks the regression of cardiac myocyte hypertrophy induced by siRNA targeting VEGFR-2, as well as $\mathrm{Cu}$-induced regression of cardiac myocyte hypertrophy.

As a whole, this body of data suggests that the VEGFR-1 pathway is somehow linked to the PKG-1 signaling pathway. It appears that both are involved in the regression of cardiac hypertrophy and are somehow linked.

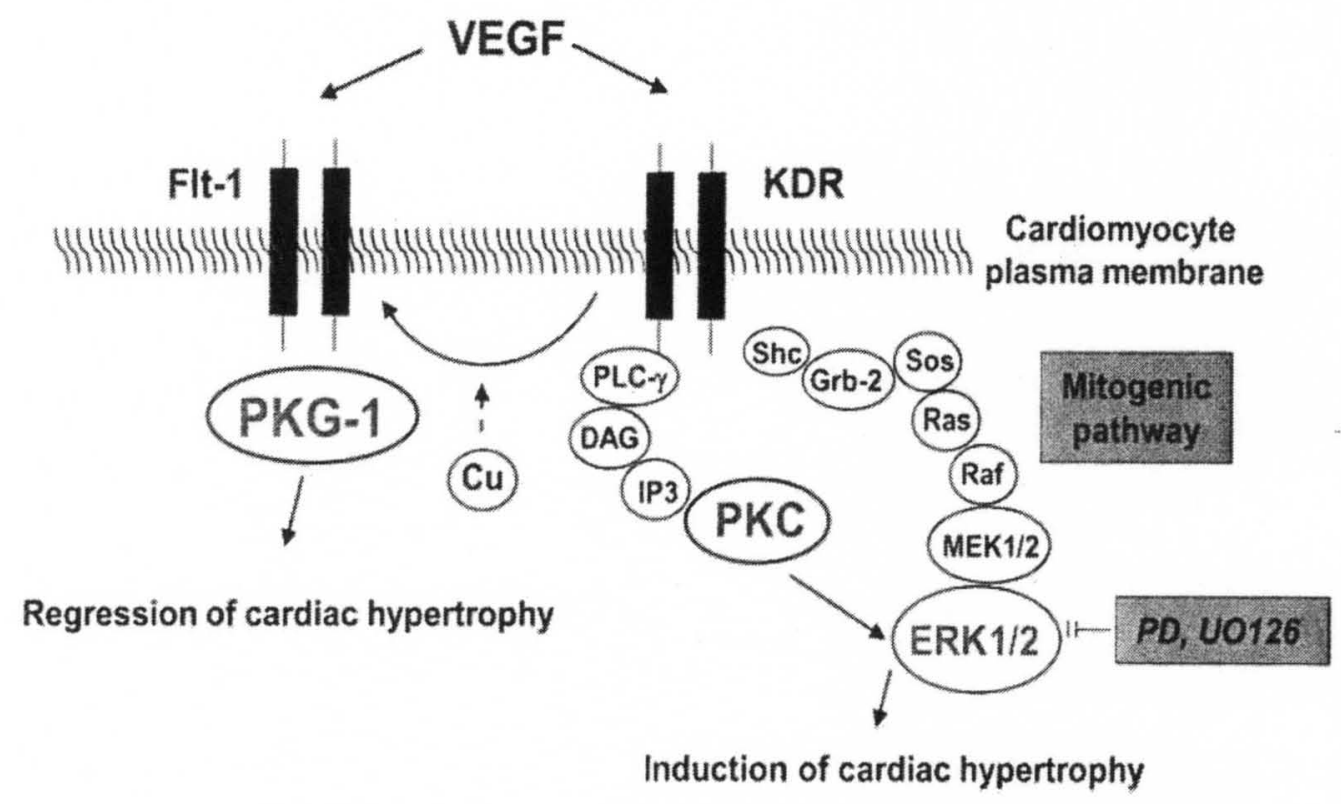

Figure 28. A complete schematic representation of VEGF receptor signaling in cardiac myocytes. 


\section{G. The involvement of Vimentin in the regression of cardiac myocyte hypertrophy}

1. The next question we asked was if there was a correlation between VEGFR-1 and PKG-1? Was it direct, or indirect? How? We then hypothesized that VEGFR-1 and PKG-1 are members of the same signaling cluster.

Copper-induced regression of cardiac myocyte hypertrophy is associated with enhanced VEGFR-1 signaling, as well as an increase in PKG-1 activity. In order to investigate the mechanisms by which this phenomena occurs, we used functional proteomics to identify proteins associated with PKG-1(Tables 1, 2 and 3) and VEGFR-1 (Table 4). There is one caviat: neither VEGFR-1 nor PKG-1 are copperbinding proteins, yet the phenomenon we have observed is related to $\mathrm{Cu}$ supplementation.

We used immunoprecipitation to pull down the immune complexes associated with VEGFR-1 as well as PKG-1. These immune complexes were sent off for LC-MS/MS analysis. Hundreds of proteins were identified in both complexes, however we were most interested in a copper-binding protein. For this reason, we chose Vimentin. Our preliminary Western blot analysis, in Figure 29 showed that PE treatment caused a decrease in the amount of Vimentin.

To further validate this interaction, we used co-immunoprecipitation, pulling down Vimentin and then subjecting the immunopreciptate to Western blot analysis. These 
experiments further validated the protein-protein interaction between Vimentin and

PKG-1 (Figure 30), and Vimentin and VEGFR-1(Figures 31 and 32). We

hypothesize that PE causes a decrease in Vimentin, and this tips the scale, favoring hypertrophic signaling and growth in cardiac myocytes. 


\section{Table 1: PKG-1 pull-down}

\author{
Control \\ $60 \mathrm{kDa}$ heat shock protein \\ mitochondrial \\ ATP synthase subunit alpha, \\ mitochondrial
}

Malate dehydrogenase, mitochondrial

14-3-3 protein beta/alpha 14-3-3 protein gamma 14-3-3 protein epsilon

14-3-3 protein zeta/delta 14-3-3 protein theta

40S ribosomal protein $S 3$

$40 S$ ribosomal protein $\mathrm{S} 12$

$40 S$ ribosomal protein $S 18$

$40 S$ ribosomal protein $S 20$

\section{$\underline{\mathrm{Cu}}$}

$60 \mathrm{kDa}$ heat shock protein, mitochondrial

ATP synthase subunit alpha, mitochondrial

ATP synthase subunit beta, mitochondrial

Malate dehydrogenase, mitochondrial

14-3-3 protein beta/alpha 14-3-3 protein gamma 14-3-3 protein epsilon 14-3-3 protein eta 14-3-3 protein zeta/delta 14-3-3 protein theta

40 S ribosomal protein $\mathrm{SA}$ $40 S$ ribosomal protein $S 2$ $40 \mathrm{~S}$ ribosomal protein $\mathrm{S} 3$ 40S ribosomal protein S5 $40 S$ ribosomal protein S6 $40 S$ ribosomal protein $S 12$ 40S ribosomal protein $S 18$
PE

$60 \mathrm{kDa}$ heat shock protein, mitochondrial

ATP synthase subunit beta, mitochondrial

Malate dehydrogenase mitochondrial

14-3-3 protein beta/alpha 14-3-3 protein gamma 14-3-3 protein epsilon 14-3-3 protein eta 14-3-3 protein zeta/delta 14-3-3 protein theta

$40 \mathrm{~S}$ ribosomal protein $\mathrm{SA}$ 40 S ribosomal protein $S 2$ $40 \mathrm{~S}$ ribosomal protein $\mathrm{S} 3$

40S ribosomal protein $\mathrm{S} 12$ $40 S$ ribosomal protein $\mathrm{S} 18$

$40 S$ ribosomal protein $\$ 20$
PE+Cu

$\mathrm{kDa}$ heat shock protein,

mitochondrial

ATP synthase subunit alpha

mitochondrial

ATP synthase subunit beta,

mitochondrial

ATP synthase subunit $O$,

mitochondrial

Malate dehydrogenase,

mitochondrial

Serine hydroxymethyltransferase,

mitochondrial

14-3-3 protein beta/alpha

14-3-3 protein gamma

14-3-3 protein epsilon

14-3-3 protein eta

14-3-3 protein zeta/delta

14-3-3 protein theta

$26 \mathrm{~S}$ proteasome non-ATPase

regulatory subunit

$40 S$ ribosomal protein $S A$

$40 S$ ribosomal protein $S 2$

$40 \mathrm{~S}$ ribosomal protein $\mathrm{S} 3$

$40 \mathrm{~S}$ ribosomal protein $\mathrm{S5}$

$40 S$ ribosomal protein $S 6$

$40 S$ ribosomal protein $S 12$

$40 \mathrm{~S}$ ribosomal protein $\mathrm{S} 18$

$40 S$ ribosomal protein $\$ 19$ 
40 S ribosomal protein $S 24$

40S ribosomal protein $\$ 28$

$40 S$ ribosomal protein $\mathrm{S} 28$

$60 S$ ribosomal protein $\mathrm{L} 4$

$60 S$ ribosomal protein $\mathrm{L} 6$

$60 S$ ribosomal protein $\mathrm{L} 17$

60S ribosomal protein L23a

$60 S$ ribosomal protein $\mathrm{L} 24$

$60 S$ ribosomal protein $\mathrm{L} 27 \mathrm{a}$

like
$60 S$ ribosomal protein L23a

60 S ribosomal protein $\mathrm{L} 7$

$60 S$ ribosomal protein $\mathrm{L} 27 \mathrm{a}$

$60 \mathrm{~S}$ acidic ribosomal protein $\mathrm{P} 0-60 \mathrm{~S}$ acidic ribosomal protein

$60 \mathrm{~S}$ acidic ribosomal protein $\mathrm{PO}$

60 S acidic ribosomal protein $\mathrm{P} 2$

$78 \mathrm{kDa}$ glucose-regulated

protein

$78 \mathrm{kDa}$ glucose-regulated protein

Protein S100-A6

Protein S100-A7

Protein S100-A7A

Protein S100-A8

Protein S100-A9

Protein S100-A11

UPF0568 protein C14orf166

Protein S100-A11

UPF0568 protein C14orf166

Alpha-actinin-1

Alpha-actinin-1
Protein S100-A11

SH3 domain-binding glutamic acid-rich-like protein

UPF0568 protein C14orf166

UPF0027 protein C22orf28

Alpha-actinin-1
40 S ribosomal protein $\$ 28$

$60 S$ ribosomal protein $\mathrm{L} A$

P0-like

$60 S$ acidic ribosomal protein $\mathrm{PO}$

40 S ribosomal protein $\$ 26$

$40 \mathrm{~S}$ ribosomal protein $\mathrm{S} 28$

$60 S$ ribosomal protein $\mathrm{LA}$

$60 S$ ribosomal protein $\mathrm{L7}$

$60 S$ ribosomal protein L1 1

$60 S$ ribosomal protein $\mathrm{L} 12$

60 S ribosomal protein $\mathrm{L} 23$

60 S ribosomal protein $\mathrm{L} 24$

$60 S$ acidic ribosomal protein $\mathrm{P} 0$

$60 S$ acidic ribosomal protein $P 1$

$60 S$ acidic ribosomal protein $\mathrm{P} 260$ S acidic ribosomal protein $\mathrm{P} 2$

$78 \mathrm{kDa}$ glucose-regulated

protein

$78 \mathrm{kDa}$ glucose-regulated protein
Protein S100-A11

SH3 domain-binding glutamic acidrich-like protein

UPF0568 protein C14orf166

UPF0027 protein C22orf28

Alpha-actinin-1

Alpha-actinin-2 
Alpha-actinin-4

Adenylyl cyclase-associated protein 1

Annexin A1

Annexin A2

Putative annexin A2-like protein Annexin A5

Actin, cytoplasmic 1

Actin, cytoplasmic 2

Actin, aortic smooth muscle Actin, alpha cardiac muscle 1 Actin, gamma-enteric smooth muscle

Actin, alpha skeletal muscle ADP-ribosylation factor 1 ADP-ribosylation factor 3 ADP-ribosylation factor 4 ADP-ribosylation factor 5

\section{ADP/ATP translocase 3}

Apolipoprotein D

ATP-dependent RNA helicase DDX3X

Beta-actin-like protein 2

Beta-actin-like protein 3

Brain acid soluble protein 1

Calmodulin
Alpha-actinin-4

Adenylyl cyclase-associated protein 1

\section{Annexin A1}

\section{Annexin A2}

Putative annexin A2-like protein

Annexin A5

Annexin A6

Actin, cytoplasmic 1

Actin, cytoplasmic 2

Actin-related protein 3

Actin, aortic smooth muscle

Actin, alpha cardiac muscle 1

Actin, gamma-enteric smooth muscle

Actin, alpha skeletal muscle ADP-ribosylation factor 1

ADP-ribosylation factor 3

ADP-ribosylation factor 4

ADP-ribosylation factor 5

ADP/ATP translocase 2

ADP/ATP translocase 3

Beta-actin-like protein 2

Beta-actin-like protein 3

Brain acid soluble protein 1

Calmodulin

Calpain small subunit 1
Alpha-actinin-4

Adenylyl cyclase-associated protein 1

Annexin A1

Annexin A2

Putative annexin A2-like

protein

Annexin A5

Annexin A6

Actin, cytoplasmic 1

Actin, cytoplasmic 2

Actin-related protein 3

Actin, aortic smooth muscle

Actin, alpha cardiac muscle 1

Actin, gamma-enteric smooth muscle

Actin, alpha skeletal muscle

ADP-ribosylation factor 1

ADP-ribosylation factor 3

ADP-ribosylation factor 4

ADP-ribosylation factor 5

Beta-actin-like protein 2

Beta-actin-like protein 3

Brain acid soluble protein 1

Calmodulin

Calpain small subunit 1
Alpha-actinin-4

Adenylyl cyclase-associated protein 1

Annexin A1

Annexin A2

Putative annexin A2-like protein

Annexin A5

Annexin A6

Actin, cytoplasmic 1

Actin, cytoplasmic 2

Actin-related protein 3

Actin, aortic smooth muscle

Actin, alpha cardiac muscle 1

Actin, gamma-enteric smooth muscle

Actin, alpha skeletal muscle

ADP-ribosylation factor 1

ADP-ribosylation factor 3

ADP-ribosylation factor 4

ADP-ribosylation factor 5

ADP/ATP translocase 1

ADP/ATP translocase 2

ADP/ATP translocase 3

Beta-actin-like protein 2

Beta-actin-like protein 3

Brain acid soluble protein 1

Calmodulin

Calpain small subunit 1 


\begin{tabular}{|c|c|c|c|}
\hline Calponin-2 & Calponin-2 & Calponin-2 & Calponin-2 \\
\hline \multirow[t]{4}{*}{ Calponin-3 } & & Calponin-3 & Calponin-3 \\
\hline & & & Calreticulin \\
\hline & & Calumenin & Calumenin \\
\hline & & Cathepsin B & Cathepsin B \\
\hline & Cathepsin D & & Cathepsin D \\
\hline \multirow[t]{3}{*}{$\begin{array}{l}\text { Chloride intracellular channel } \\
\text { protein } 1\end{array}$} & $\begin{array}{l}\text { Chloride intracellular channel } \\
\text { protein } 1 \\
\text { Chloride intracellular channel } \\
\text { protein } 4\end{array}$ & $\begin{array}{l}\text { Chloride intracellular channel } \\
\text { protein } 1\end{array}$ & $\begin{array}{l}\text { Chloride intracellular channel protein } \\
1 \\
\text { Chloride intracellular channel protein } \\
4\end{array}$ \\
\hline & Clathrin heavy chain 1 & Clathrin heavy chain 1 & Clathrin heavy chain 1 \\
\hline & Coatomer subunit beta & & \\
\hline Cofilin-1 & Cofilin-1 & Cofilin-1 & Cofilin-1 \\
\hline Cofilin-2 & & Cofilin-2 & , \\
\hline \multicolumn{4}{|l|}{ Complement C3 } \\
\hline \multicolumn{4}{|l|}{ Complement C4-A } \\
\hline \multirow{3}{*}{$\begin{array}{l}\text { Complement C4-B } \\
\text { Cullin-associated NEDD8- } \\
\text { dissociated protein } 1\end{array}$} & & & \\
\hline & $\begin{array}{l}\text { Cullin-associated NEDD8- } \\
\text { dissociated protein } 1\end{array}$ & & $\begin{array}{l}\text { Cullin-associated NEDD8- } \\
\text { dissociated protein } 1\end{array}$ \\
\hline & & Cytochrome c & \\
\hline \multirow{3}{*}{$\begin{array}{l}\text { Elongation factor } 1 \text {-alpha } 1 \\
\text { Putative elongation factor } 1 \text { - } \\
\text { alpha-like } 3\end{array}$} & Elongation factor 1 -alpha 1 & Elongation factor 1-alpha 1 & Elongation factor 1 -alpha 1 \\
\hline & $\begin{array}{l}\text { Putative elongation factor } 1 \text { - } \\
\text { alpha-like } 3\end{array}$ & $\begin{array}{l}\text { Putative elongation factor } 1 \text { - } \\
\text { alpha-like } 3\end{array}$ & $\begin{array}{l}\text { Putative elongation factor } 1 \text {-alpha- } \\
\text { like } 3\end{array}$ \\
\hline & Elongation factor 1 -beta & Elongation factor 1-beta & Elongation factor 1-beta \\
\hline Elongation factor 2 & Elongation factor 2 & Elongation factor 2 & Elongation factor 2 \\
\hline Elongation factor 1-alpha 2 & Elongation factor 1-alpha 2 & Elongation factor 1-alpha 2 & Elongation factor 1-alpha 2 \\
\hline \multirow[t]{2}{*}{ Elongation factor 1-delta } & Elongation factor 1-delta & Elongation factor 1-delta & Elongation factor 1-delta \\
\hline & Elongation factor 1-gamma & Elongation factor 1-gamma & Elongation factor 1-gamma \\
\hline Endoplasmin & Endoplasmin & Endoplasmin & Endoplasmin \\
\hline Alpha-enolase & Alpha-enolase & Alpha-enolase & Alpha-enolase \\
\hline Beta-enolase & Beta-enolase & Beta-enolase & Beta-enolase \\
\hline Gamma-enolase & Gamma-enolase & Gamma-enolase & Gamma-enolase \\
\hline
\end{tabular}


Eukaryotic initiation factor 4A-I

Eukaryotic initiation factor 4A-II

Eukaryotic translation initiation

factor 5A-1

Eukaryotic translation initiation

factor 5A-1-like

Eukaryotic translation initiation

factor $5 \mathrm{~A}-2$

Exportin-2
Eukaryotic initiation factor 4A-I Eukaryotic initiation factor 4A-

Eukaryotic translation initiation factor $5 \mathrm{~A}-1$

Eukaryotic translation initiation

factor 5A-1-like

Eukaryotic translation initiation

factor 5A-2

Exportin-2

F-actin-capping protein subunit alpha-1

Filamin-A

Fructose-bisphosphate aldolase

Fructose-bisphosphate aldolase A A

Galectin-1

Glucose-6-phosphate isomerase

Glutathione S-transferase $\mathbf{P}$

Glyceraldehyde-3-phosphate

dehydrogenase

GTP-binding nuclear protein Ran

Heat shock protein HSP 90-alpha Heat shock protein HSP 90-beta

Heat shock-related $70 \mathrm{kDa}$ protein

\section{Galectin-1}

Glucose-6-phosphate isomerase

Glutathione S-transferase P Glyceraldehyde-3-phosphate dehydrogenase

Ran

Heat shock protein HSP 90-

alpha

Heat shock protein HSP 90-beta

Heat shock protein beta-1

Heat shock-related $70 \mathrm{kDa}$

protein 2

\section{GTP-binding nuclear protein}

Eukaryotic initiation factor 4A-I Eukaryotic initiation factor $4 \mathrm{~A}$ II

Eukaryotic translation initiation factor 5A-1

Eukaryotic translation initiation factor 5A-1-like

Eukaryotic translation initiation factor $5 \mathrm{~A}-2$

(2)

Fructose-bisphosphate aldolase A

Galectin-1

Glucose-6-phosphate isomerase Glutathione S-transferase omega-1

Glutathione S-transferase P

Glyceraldehyde-3-phosphate

dehydrogenase

Guanine nucleotide-binding

protein subunit beta-2-1

GTP-binding nuclear protein

Ran

Heat shock protein HSP 90alpha

Heat shock protein HSP 90-beta

Heat shock protein beta-1

Heat shock-related $70 \mathrm{kDa}$

protein 2
Eukaryotic initiation factor $4 \mathrm{~A}-\mathrm{I}$

Eukaryotic initiation factor 4A-II

Eukaryotic translation initiation

factor 5A-1

Eukaryotic translation initiation

factor 5A-1-like

Eukaryotic translation initiation factor 5A-2

\section{Ezrin}

F-actin-capping protein subunit alpha-1

Filamin-A

Filamin-B

Filamin-C

Fructose-bisphosphate aldolase A

Galectin-1 .

Glucose-6-phosphate isomerase

Glutathione S-transferase P

Glyceraldehyde-3-phosphate

dehydrogenase

GTP-binding nuclear protein Ran

Heat shock protein HSP 90-alpha

Heat shock protein HSP 90-beta

Heat shock-related $70 \mathrm{kDa}$ protein 2 
Heat shock cognate $71 \mathrm{kDa}$

protein

Putative heat shock protein HSP

90-alpha A2

Putative heat shock protein HSP

90-beta 2

Putative heat shock protein HSP

90-beta-3

Heterogeneous nuclear

ribonucleoproteins A2/B1

Heterogeneous nuclear ribonucleoproteins $\mathrm{C} 1 / \mathrm{C} 2$

Heterogeneous nuclear ribonucleoprotein $\mathrm{K}$

\section{Heat shock $70 \mathrm{kDa}$ protein 6}

Heat shock $70 \mathrm{kDa}$ protein 6

Heat shock cognate $71 \mathrm{kDa}$

protein

Putative heat shock protein HSP 90-alpha A2

Putative heat shock protein HSP

90-beta 2

Putative heat shock protein HSP

90-beta-3

Heterogeneous nuclear ribonucleoprotein $\mathrm{Al}$

Putative heterogeneous nuclear ribonucleoprotein $\mathrm{A}$

Heterogeneous nuclear ribonucleoprotein Al-like

Heterogeneous nuclear

ribonucleoprotein $\mathrm{A} / \mathrm{B}$

Heterogeneous nuclear

ribonucleoproteins $\mathrm{A} 2 / \mathrm{B} 1$

Heterogeneous nuclear ribonucleoproteins $\mathrm{C} 1 / \mathrm{C} 2$

Heterogeneous nuclear

ribonucleoprotein D0

Heterogeneous nuclear ribonucleoprotein $\mathrm{K}$
Heat shock cognate $71 \mathrm{kDa}$ protein

Putative heat shock protein HSP 90-alpha A2

Putative heat shock protein HSP

90-beta 2

Putative heat shock protein HSP

90-beta-3

Putative heat shock protein HSP

90-beta 4

Heterogeneous nuclear

ribonucleoprotein A1

Putative heterogeneous nuclear

ribonucleoprotein A

Heterogeneous nuclear

ribonucleoprotein A1-like

Heterogeneous nuclear

ribonucleoproteins A2/B1

Heterogeneous nuclear

ribonucleoprotein C-like

Heterogeneous nuclear

ribonucleoproteins $\mathrm{C} 1 / \mathrm{C} 2$

Heterogeneous nuclear

ribonucleoprotein D0

Heterogeneous nuclear

ribonucleoprotein $\mathrm{K}$

Heterogeneous nuclear

ribonucleoprotein $\mathrm{M}$

Heat shock $70 \mathrm{kDa}$ protein 1

Heat shock $70 \mathrm{kDa}$ protein 6

Putative heat shock $70 \mathrm{kDa}$ protein 7

Heat shock $70 \mathrm{kDa}$ protein $1 \mathrm{~L}$

Heat shock cognate $71 \mathrm{kDa}$ protein

Putative heat shock protein HSP 90-

alpha A2

Phock protein HSP 90-

beta 2

Putative heat shock protein HSP 90-

beta- 3

Putative heat shock protein HSP 90-

beta 4

Heterogeneous nuclear

ribonucleoprotein Al

Putative heterogeneous nuclear

ribonucleoprotein $\mathrm{A}$

Heterogeneous nuclear

ribonucleoprotein A1-like

Heterogeneous nuclear

ribonucleoproteins A2/B

Heterogeneous nuclear

ribonucleoprotein C-like 1

Heterogeneous nuclear

ribonucleoproteins $\mathrm{C} 1 / \mathrm{C} 2$

Heterogeneous nuclear

ribonucleoprotein D0

Heterogeneous nuclear

ribonucleoprotein D-like

Heterogeneous nuclear

ribonucleoprotein $\mathrm{K}$ 
Importin subunit beta-1

L-lactate dehydrogenase A chain

L-lactate dehydrogenase $B$ chain Lysosome-associated membrane glycoprotein 2

Myosin-9
Myosin-10
Myosin-11
Myosin-14
Myosin light polypeptide 6
Myosin light chain 6B
Myosin regulatory light chain
12A
Myosin regulatory light chain
12B

Histone H2A type 1

Histone H2A type 1-B/E

Histone $\mathrm{H} 2 \mathrm{~A}$ type 1-C

Histone H2A type 1-D

Histone H2A type 1-H

Histone H2A type 1-J

Histone H2A type 2-A

Histone H2A type 2-C

Histone H2A type 3

Histone H2A.J

Importin-5

Importin subunit alpha-2

Importin subunit beta-1

\section{Latexin}

L-lactate dehydrogenase $B$ chain

Lysosome-associated membrane glycoprotein 2

Moesin
Myosin-9
Myosin-10
Myosin-11
Myosin-14
Myosin light polypeptide 6
Myosin light chain 6B
Myosin regulatory light chain
12A
Myosin regulatory light chain
12B

Histone H2A type 1

Histone $\mathrm{H} 2 \mathrm{~A}$ type 1-B/E

Histone $\mathrm{H} 2 \mathrm{~A}$ type 1-C

Histone H2A type 1-D

Histone $\mathrm{H} 2 \mathrm{~A}$ type 1-H

Histone $\mathrm{H} 2 \mathrm{~A}$ type 1 -J

Histone H2A type 2-A

Histone $\mathrm{H} 2 \mathrm{~A}$ type 2-C

Histone H2A type 3

Histone H2A.J

Importin subunit beta-1

Latexin

L-lactate dehydrogenase $\mathrm{A}$

chain

L-lactate dehydrogenase $B$

chain

Moesin

Myosin-9

Myosin-11

Myosin-14

Myosin light polypeptide 6

Myosin light chain 6B

Myosin regulatory light chain $12 \mathrm{~A}$

Myosin regulatory light chain

12B
Importin-5

Importin subunit beta- 1

Integrin beta-1

Latexin

L-lactate dehydrogenase A chain

L-lactate dehydrogenase $B$ chain

Malate dehydrogenase, cytoplasmic Moesin

Myosin-9

Myosin-10

Myosin-1 1

Myosin-14

Myosin light polypeptide 6

Myosin light chain 6B

Myosin regulatory light chain $12 \mathrm{~A}$

Myosin regulatory light chain 12B 
Myosin regulatory light polypeptide 9

Myristoylated alanine-rich Ckinase substrate

Nicotinamide N-

methyltransferase

Neuroblast differentiation-

associated protein AHNAK
Myosin regulatory light

polypeptide 9

Myristoylated alanine-rich C-

kinase substrate

Nicotinamide $\mathrm{N}$ -

methyltransferase

Neuroblast differentiation-

associated protein AHNAK

Nucleophosmin

POTE ankyrin domain family member $\mathrm{E}$

POTE ankyrin domain family member $\mathbf{F}$

Peptidyl-prolyl cis-trans

isomerase $\mathrm{A}$

Peptidyl-prolyl cis-trans

isomerase $B$

Peroxiredoxin-1

Peroxiredoxin-6

Peripherin

Phosphoglycerate kinase 1

Phosphoglycerate mutase 1
Myosin regulatory light

polypeptide 9

Myristoylated alanine-rich C-

kinase substrate

Nicotinamide $\mathrm{N}$ -

methyltransferase

Neuroblast differentiation-

associated protein AHNAK

Nucleophosmin

Putative nucleoside diphosphate

kinase

Nucleoside diphosphate kinase

POTE ankyrin domain family member E

POTE ankyrin domain family member $\mathrm{F}$

Peptidyl-prolyl cis-trans

isomerase A

Peptidyl-prolyl cis-trans

isomerase B

Peroxiredoxin-1

Peroxiredoxin-6

Peripherin

Phosphoglycerate kinase 1

Phosphoglycerate mutase 1

Phosphoglycerate mutase 2

Probable phosphoglycerate

mutase 4
Myosin regulatory light polypeptide 9

Myristoylated alanine-rich C-kinase substrate

Multifunctional protein ADE2

$\mathrm{NAD}(\mathrm{P}) \mathrm{H}$ dehydrogenase [quinone]

Nicotinamide $\mathrm{N}$-methyltransferase

Neuroblast differentiation-associated protein AHNAK

Nucleophosmin

Nuclease-sensitive element-binding protein 1

POTE ankyrin domain family member $\mathrm{E}$

POTE ankyrin domain family member $\mathrm{F}$

Peptidyl-prolyl cis-trans isomerase A

Peptidyl-prolyl cis-trans isomerase B Peroxiredoxin-1

\section{Peripherin}

Phosphoglycerate kinase 1

Phosphoglycerate mutase 1

Phosphoglycerate mutase 2

Probable phosphoglycerate mutase 4

Proteasome subunit beta type- 3 


\section{Plastin-3}

Proactivator polypeptide

Profilin-1

Prolactin-inducible protein

Proteasome activator complex subunit 2

Protein disulfide-isomerase

Protein disulfide-isomerase A3

Pyruvate kinase isozymes M1/M2
Ras-related protein Rab-10

Reticulon-4
Plastin-3

Polyadenylate-binding protein 1

Proactivator polypeptide

Profilin-1

Protein disulfide-isomerase

Protein disulfide-isomerase A3

Protein disulfide-isomerase A6

Pyruvate kinase isozymes

\section{M1/M2}

Poly(rC)-binding protein 1

Poly(rC)-binding protein 2

Rab GDP dissociation inhibitor alpha

Rab GDP dissociation inhibitor

beta
Reticulon-4
Plastin-3

Proactivator polypeptide

Profilin- 1

Protein disulfide-isomerase

Protein disulfide-isomerase A3

Protein disulfide-isomerase A4

Protein disulfide-isomerase A6

Pyruvate kinase isozymes

\section{M1/M2}

Poly(rC)-binding protein 1

Poly(rC)-binding protein 2

Poly(rC)-binding protein 3

Ras-related protein Rab-10

Ras-related protein Rab-1A

Ras-related protein Rab-1B

Ras-related protein Rab-15

Putative Ras-related protein

Rab-1C

Ras-related protein Rab-10

Ras-related protein Rab-1B

Ras-related protein Rab-15
Proactivator polypeptide

Profilin-1

Protein disulfide-isomerase

Protein disulfide-isomerase A3

Protein disulfide-isomerase A6

Pyruvate kinase isozymes M1/M2

Poly(rC)-binding protein 1

Poly(rC)-binding protein 2

Rab GDP dissociation inhibitor alpha

Rab GDP dissociation inhibitor beta Radixin

Ras-related protein Rab-5C

Ras-related protein Rab-10

Ras-related protein Rab-1A

Ras-related protein Rab-1B

Ras-related protein Rab-15

Putative Ras-related protein Rab-1C Ras-related C3 botulinum toxin substrate

Ras-related $\mathrm{C} 3$ botulinum toxin substrate 3

Reticulon-4 
1

RNA-binding protein EWS

RNA-binding protein FUS

Sacsin

Serpin $\mathbf{H} 1$

T-complex protein 1 subunit beta T-complex protein 1 subunit gamma
Rho GDP-dissociation inhibitor

Ribonuclease inhibitor

RNA-binding protein FUS

\section{Serpin H1 \\ Serine/threonine-protein \\ phosphatase $2 \mathrm{~A} 65 \mathrm{kDa}$}

T-complex protein 1 subunit

beta

T-complex protein 1 subunit

gamma

T-complex protein 1 subunit

theta

T-complex protein 1 subunit eta

Thioredoxin

Thioredoxin domain-containing protein 5

Transgelin

Transgelin-2

Transitional endoplasmic

reticulum ATPase

\section{Transketolase}

Triosephosphate isomerase

Tubulin alpha-1A chain

Tubulin alpha-1B chain

Tubulin alpha- $1 \mathrm{C}$ chain
Triosephosphate isomerase

Tropomyosin alpha-4 chain

Tubulin alpha-1 A chain

Tubulin alpha-1B chain

Tubulin alpha-1C chain
Serpin H1

T-complex protein 1 subunit

beta

T-complex protein 1 subunit gamma

T-complex protein 1 subunit theta

\section{Thioredoxin}

Transgelin

Transgelin-2

Transketolase

Triosephosphate isomerase

Tropomyosin alpha-4 chain

Tubulin alpha-1A chain

Tubulin alpha-1B chain

Tubulin alpha-1C chain
Serpin H1

Serine/threonine-protein phosphatase $2 \mathrm{~A} 65 \mathrm{kDa}$

Sorcin

T-complex protein 1 subunit beta

$\mathrm{T}$-complex protein 1 subunit gamma T-complex protein 1 subunit delta

T-complex protein 1 subunit theta

T-complex protein 1 subunit eta

T-complex protein 1 subunit epsilon

Transgelin

Transgelin-2

Translationally-controlled tumor protein

Transketolase

Triosephosphate isomerase

Tubulin alpha-1 A chain

Tubulin alpha-1B chain

Tubulin alpha-1C chain 
Tubulin alpha chain-like 3 Tubulin alpha-3C/D chain Tubulin alpha-3E chain Tubulin alpha-4A chain Tubulin alpha- 8 chain

Tubulin beta chain

Tubulin beta- $2 \mathrm{C}$ chain

Tubulin beta- 8 chain

Tubulin beta- 8 chain $B$

Putative tubulin beta- $4 \mathrm{q}$ chain

Tubulin beta-2A chain

Tubulin beta-2B chain

Tubulin beta- 3 chain Putative tubulin-like protein alpha-4B

Tubulin alpha chain-like 3

\section{Ubiquitin \\ Ubiquitin carboxyl-terminal hydrolase isozyme $\mathrm{Ll}$}

Ubiquitin-conjugating enzyme E2

$\mathrm{N}$

Vimentin

Zinc-alpha-2-glycoprotein
Tubulin alpha chain-like 3

Tubulin alpha-3C/D chain

Tubulin alpha-3E chain

Tubulin alpha-4A chain

Tubulin alpha-8 chain

Tubulin beta chain

Tubulin beta- 1 chain

Tubulin beta- $2 \mathrm{C}$ chain

Tubulin beta- 4 chain

Tubulin beta- 8 chain

Tubulin beta- 8 chain B

Putative tubulin beta-4q chain

Tubulin beta-2A chain

Tubulin beta-2B chain

Tubulin beta- 3 chain

Tubulin beta- 6 chain

Putative tubulin-like protein

alpha-4B

Ubiquitin

Ubiquitin carboxyl-terminal

hydrolase isozyme L1
Tubulin alpha chain-like 3

Tubulin alpha-3C/D chain

Tubulin alpha-3E chain

Tubulin alpha-4A chain

Tubulin alpha- 8 chain

Tubulin beta chain

Tubulin beta- 1 chain

Tubulin beta- $2 \mathrm{C}$ chain

Tubulin beta- 4 chain

Tubulin beta- 8 chain

Tubulin beta- 8 chain $B$

Putative tubulin beta- $4 \mathrm{q}$ chain

Tubulin beta- $2 \mathrm{~A}$ chain

Tubulin beta- $2 \mathrm{~B}$ chain

Tubulin beta- 3 chain

Tubulin beta- 6 chain

Putative tubulin-like protein

alpha-4B

Ubiquitin carboxyl-terminal hydrolase isozyme Ll
Tubulin alpha chain-like 3

Tubulin alpha-3C/D chain

Tubulin alpha-3E chain

Tubulin alpha-4A chain

Tubulin alpha-8 chain

Tubulin beta chain

Tubulin beta-2 $\mathrm{C}$ chain

Tubulin beta- 4 chain

Tubulin beta- 8 chain

Tubulin beta- 8 chain B

Putative tubulin beta- $4 \mathrm{q}$ chain

Tubulin beta-2A chain

Tubulin beta-2B chain

Tubulin beta- 3 chain

Tubulin beta- 6 chain

Putative tubulin-like protein alpha-

4B

Ubiquitin carboxyl-terminal

hydrolase isozyme $\mathrm{Ll}$

Ubiquitin-conjugating enzyme E2

variant 1

Ubiquitin-conjugating enzyme E2

variant 2

Vimentin
Vimentin

Vimentin 
Table 2: PKG-1 pull-down

\section{Control}

$10 \mathrm{kDa}$ heat shock protein mitochondrial

$60 \mathrm{kDa}$ heat shock protein,

mitochondrial

Stress-70 protein, mitochondrial ATP synthase subunit alpha, mitochondrial

ATP synthase subunit beta, mitochondrial

Malate dehydrogenase,

mitochondrial
14-3-3 protein beta/alpha 14-3-3 protein zeta/delta 14-3-3 protein gamma 14-3-3 protein theta 14-3-3 protein eta

14-3-3 protein epsilon 14-3-3 protein sigma 40 S ribosomal protein SA $40 \mathrm{~S}$ ribosomal protein $\mathrm{S} 2$ $40 \mathrm{~S}$ ribosomal protein $\mathrm{S} 3$ 40 S ribosomal protein $\$ 4, X$ isoform

$40 \mathrm{~S}$ ribosomal protein $\mathrm{S} 6$ $40 S$ ribosomal protein $S 8$ $40 S$ ribosomal protein $\$ 9$ $40 S$ ribosomal protein $S 12$ $40 S$ ribosomal protein $S 15 a$ $40 S$ ribosomal protein $S 18$

\section{PE}

$10 \mathrm{kDa}$ heat shock protein, mitochondrial

$60 \mathrm{kDa}$ heat shock protein,

mitochondrial

ATP synthase subunit alpha, mitochondrial

ATP synthase subunit beta, mitochondrial

Malate dehydrogenase, mitochondria

Thioredoxin-dependent peroxide reductase, mitochon

14-3-3 protein beta/alpha

14-3-3 protein zeta/delta

14-3-3 protein gamma

14-3-3 protein theta

14-3-3 protein eta

14-3-3 protein epsilon

14-3-3 protein sigma

$40 \mathrm{~S}$ ribosomal protein $\mathrm{SA}$

$40 \mathrm{~S}$ ribosomal protein $\mathrm{S} 2$

$40 S$ ribosomal protein $S 3$

$40 S$ ribosomal protein $S 4, X$

isoform

$40 \mathrm{~S}$ ribosomal protein $\mathrm{S} 8$

$40 S$ ribosomal protein $S 9$

$40 S$ ribosomal protein $S 12$

40S ribosomal protein $S 18$

40S ribosomal protein $S 20$

\section{$\underline{\mathrm{Cu}}$}

$60 \mathrm{kDa}$ heat shock protein,

mitochondrial

Stress-70 protein

mitochondrial

ATP synthase subunit alpha,

mitochondrial

Malate dehydrogenase,

mitochondrial

Thioredoxin-dependent

peroxide reductase

mitochon

14-3-3 protein beta/alpha

14-3-3 protein zeta/delta

14-3-3 protein gamma

14-3-3 protein theta

14-3-3 protein eta

14-3-3 protein epsilon

14-3-3 protein sigma

40 S ribosomal protein $\mathrm{SA}$

40 S ribosomal protein $S 3$

40S ribosomal protein $\mathrm{S} 4, \mathrm{X}$ isoform

40 S ribosomal protein $S 8$ 40 S ribosomal protein $S 9$ 40 S ribosomal protein $\mathrm{S} 12$

40S ribosomal protein $S 19$

$40 S$ ribosomal protein $\$ 20$

\section{$\underline{P E}+\mathrm{Cu}$}

$10 \mathrm{kDa}$ heat shock protein, mitochondrial

$60 \mathrm{kDa}$ heat shock protein, mitochondrial

Stress-70 protein, mitochondrial

ATP synthase subunit alpha, mitochondrial

ATP synthase subunit beta, mitochondria

Malate dehydrogenase, mitochondria

14-3-3 protein beta/alpha

14-3-3 protein zeta/delta

14-3-3 protein gamma

14-3-3 protein theta

14-3-3 protein eta

14-3-3 protein epsilon

14-3-3 protein sigma

40 S ribosomal protein SA

$40 S$ ribosomal protein $\mathrm{S} 2$

$40 \mathrm{~S}$ ribosomal protein $\mathrm{S} 3$

$40 S$ ribosomal protein $S 4, X$ isoform

$40 S$ ribosomal protein $S 9$

$40 S$ ribosomal protein $S 12$

40S ribosomal protein $\mathrm{S} 18$

40S ribosomal protein $S 19$

$40 \mathrm{~S}$ ribosomal protein $\mathrm{S} 20$ 
40S ribosomal protein S25

60 S ribosomal protein L6

$60 \mathrm{~S}$ ribosomal protein $\mathrm{L} 7$

60 S ribosomal protein $\mathrm{L} 12$

$60 \mathrm{~S}$ ribosomal protein $\mathrm{L} 13$

$60 S$ ribosomal protein L15

60 S ribosomal protein $\mathrm{L} 22$

60 S ribosomal protein L30

$60 S$ ribosomal protein L36

60 S acidic ribosomal protein $\mathrm{PO}$

$60 S$ acidic ribosomal protein $\mathrm{P} 2$

$78 \mathrm{kDa}$ glucose-regulated protein A-kinase anchor protein 12

ADP/ATP translocase 2

ADP/ATP translocase 3

ADP-ribosylation factor 1

ADP-ribosylation factor 3

ATP-citrate synthase

ATP-dependent RNA helicase

DDX39

ATP-dependent RNA helicase

DDX3X

ATP-dependent RNA helicase

\section{DDX3Y}

Actin, alpha cardiac muscle 1
60S ribosomal protein L6 $60 \mathrm{~S}$ ribosomal protein $\mathrm{L} 7$

60S ribosomal protein L12 60 S ribosomal protein $\mathrm{L} 13$ 60 S ribosomal protein $\mathrm{L} 14$

$60 S$ ribosomal protein $\mathrm{L} 18$ $60 S$ ribosomal protein $\mathrm{L} 22$

$60 \mathrm{~S}$ ribosomal protein $\mathrm{L} 30$

$60 S$ acidic ribosomal protein $\mathrm{P} 0$

$60 S$ acidic ribosomal protein $P 2$ $78 \mathrm{kDa}$ glucose-regulated protein

ADP/ATP translocase 1

ADP/ATP translocase 2

ADP/ATP translocase 3

ADP-ribosylation factor 1

ADP-ribosylation factor 3

ADP-ribosylation factor 4

ADP-ribosylation factor 5

Probable ATP-dependent RNA helicase DDX 17

Probable ATP-dependent RNA helicase DDX5

ATP-dependent RNA helicase

DDX39

ATP-dependent RNA helicase

DDX3X

Actin, alpha cardiac muscle 1
40 S ribosomal protein $\$ 25$

60S ribosomal protein $\mathrm{L} 7$

$60 S$ ribosomal protein $\mathrm{L} 10 \mathrm{a}$

60 S ribosomal protein $\mathrm{L} 12$

$60 S$ ribosomal protein $\mathrm{L} 13$

60 S ribosomal protein L14

60 S ribosomal protein $\mathrm{L} 22$

$60 S$ ribosomal protein $\mathrm{L} 30$

$60 \mathrm{~S}$ acidic ribosomal protein

$\mathrm{P} 2$

$78 \mathrm{kDa}$ glucose-regulated protein

$60 S$ acidic ribosomal protein $\mathrm{P} 2$

$78 \mathrm{kDa}$ glucose-regulated protein

A-kinase anchor protein 12

ADP/ATP translocase 3

ADP-ribosylation factor 1

ADP-ribosylation factor 3

ADP-ribosylation factor 4

ADP-ribosylation factor 1

ADP-ribosylation factor 3

ADP-ribosylation factor 4

ADP-ribosylation factor 5

Probable ATP-dependent

RNA helicase DDX17

Probable ATP-dependent

RNA helicase DDX5

Probable ATP-dependent RNA helicase DDX5

\section{ATP-dependent RNA}

helicase DDX3X

ATP-dependent RNA

helicase DDX3Y

Actin, alpha cardiac muscle Actin, alpha cardiac muscle 1 
Actin, aortic smooth muscle Actin, alpha skeletal muscle Actin, gamma-enteric smooth muscle

Actin, cytoplasmic 1

Actin, cytoplasmic 2

Adenylyl cyclase-associated

protein 1

Adenosylhomocysteinase

Alpha-actinin-1

Alpha-actinin-2

Alpha-actinin-3

Alpha-actinin-4

Annexin A1

Annexin A2

Annexin A5

Annexin A6

\section{Calmodulin}

Calnexin

Calponin-2

Calreticulin

Chloride intracellular channel

\section{protein}

Chloride intracellular channel

protein 4

Clathrin heavy chain 1

Clathrin heavy chain 2

Coatomer subunit gamma

Cofilin-1

Cofilin-2

Complement component $1 \mathrm{Q}$

subcomponent-binding prot

Cytoskeleton-associated protein 4
1

Actin, aortic smooth muscle Actin, alpha skeletal muscle

Actin, gamma-enteric smooth muscle

Actin, cytoplasmic 1

Actin, cytoplasmic 2

Adenylyl cyclase-associated

protein 1

Adenosylhomocysteinase

Alpha-actinin-1

Alpha-actinin-4

Annexin A1

Annexin A2

Annexin A5

Annexin A6

Calmodulin

Calponin-2

Calreticulin

Cathepsin D

Chloride intracellular channel

protein 1

Chloride intracellular channel

protein 4

Clathrin heavy chain I

Clathrin heavy chain 2

\section{Cofilin-1}

Cofilin-2

Complement component $1 \mathrm{Q}$ subcomponent-binding prot

Cytoskeleton-associated protein
Actin, aortic smooth muscle Actin, alpha skeletal muscle Actin, gamma-enteric smooth muscle Actin, cytoplasmic 1

Actin, cytoplasmic 2 Adenylyl cyclase-associated protein 1

Alpha-actinin-1

Alpha-actinin-2

Alpha-actinin-4

Annexin A1

Annexin A2

Annexin A5

Annexin A6

Calmodulin

Calreticulin

Chloride intracellular

channel protein 4

Clathrin heavy chain 1

Clathrin heavy chain 2

\section{Cofilin-1}

Complement component 1

Q subcomponent-binding

prot

Cytoskeleton-associated
Actin, aortic smooth muscle Actin, alpha skeletal muscle

Actin, gamma-enteric smooth muscle Actin, cytoplasmic 1

Actin, cytoplasmic 2

Adenylyl cyclase-associated protein 1

Adenosylhomocysteinase

Alpha-actinin-1

Alpha-actinin-4

Annexin A1

Annexin A2

Annexin A5

Annexin A6

Annexin A7

Calmodulin

Calponin-2

Calreticulin

Chloride intracellular channel protein 1

Clathrin heavy chain 1

Clathrin heavy chain 2

Cofilin-1

Cofilin-2

Cytoskeleton-associated protein 4 


\section{Cystatin-B}

Desmin

Dolichyl-

diphosphooligosaccharide--protein

glycosyl

Elongation factor 1-alpha 1

Elongation factor 1-alpha 2

Elongation factor 1-gamma

Elongation factor 1-delta

Elongation factor 2

Endoplasmin

Alpha-enolase

Beta-enolase

Eukaryotic initiation factor 4A-I

Eukaryotic initiation factor 4A-II Eukaryotic translation initiation

factor 5A-1

Eukaryotic translation initiation

factor 5A-2

Ezrin

F-actin-capping protein subunit

alpha-1

Fascin

Filamin-A

Filamin-B

Filamin-C

Fructose-bisphosphate aldolase A Galectin-1

Glucose-6-phosphate 1-

dehydrogenase
Cystatin-B

Desmin

Elongation factor 1-alpha 1

Elongation factor 1-alpha 2

Elongation factor 1-beta

Elongation factor 1-gamma

Elongation factor 2

Endoplasmin

Alpha-enolase

Beta-enolase

Gamma-enolase

Eukaryotic initiation factor $4 \mathrm{~A}-\mathrm{I}$

Eukaryotic initiation factor $4 \mathrm{~A}$ -

II

Eukaryotic translation initiation

factor 5A-1

Eukaryotic translation initiation

factor 5A-2

Ezrin

rotein 4

Cystatin-B

Cytochrome c

Desmin

Cystatin-B

Desmin

Elongation factor 1-alpha 1

Elongation factor 1-alpha 2

Elongation factor 1-beta

Elongation factor 1-gamma

Elongation factor 2

Endoplasmin

Alpha-enolase

Beta-enolase

Gamma-enolase

Eukaryotic initiation factor

\section{A-I}

Eukaryotic initiation factor

4A-II

Eukaryotic translation

initiation factor $5 \mathrm{~A}-1$

Eukaryotic translation

initiation factor $5 \mathrm{~A}-2$

Ezrin

Filamin-A

Filamin-A

Filamin-B

Filamin-C

Fructose-bisphosphate aldolase

A

Galectin-1

Fructose-bisphosphate aldolase A

Glucose-6-phosphate 1-

dehydrogenase
Elongation factor 1-alpha 1

Elongation factor 1-alpha 2

Elongation factor 1-beta

Elongation factor 1-gamma

Elongation factor 1-delta

Elongation factor 2

Endoplasmin

Alpha-enolase

Beta-enolase

Eukaryotic initiation factor 4A-I

Eukaryotic initiation factor 4A-II

Eukaryotic translation initiation factor 5A-1

Ezrin

Filamin-A

Filamin-B

Filamin-C

Fructose-bisphosphate aldolase A

Galectin-1 
Glucose-6-phosphate isomerase Glucosidase 2 subunit beta

Glutathione S-transferase P

Glutathione S-transferase omega-1

Glyceraldehyde-3-phosphate

dehydrogenase

Guanine nucleotide-binding protein subunit beta-2-1

Heat shock cognate $71 \mathrm{kDa}$ protein

Heat shock $70 \mathrm{kDa}$ protein $1 \mathrm{~A} / 1 \mathrm{~B}$

Heat shock $70 \mathrm{kDa}$ protein 4 Heat shock $70 \mathrm{kDa}$ protein 6

Hsp90 co-chaperone Cdc37

Heat shock protein HSP 90-alpha

Heat shock protein HSP 90-beta

Heat shock-related $70 \mathrm{kDa}$ protein 2

Heterogeneous nuclear ribonucleoprotein Al

Heterogeneous nuclear

ribonucleoprotein A/B

Heterogeneous nuclear

ribonucleoproteins A2/B1

Heterogeneous nuclear

ribonucleoprotein $\mathrm{A} 3$
Glucose-6-phosphate

Glucose-6-phosphate isomerase

Glucosidase 2 subunit beta

Glutathione S-transferase P

Glutathione S-transferase

omega-1

Glyceraldehyde-3-phosphate dehydrogenase

Guanine nucleotide-binding

protein subunit beta-2-1

GTP-binding nuclear protein

Ran

Heat shock cognate $71 \mathrm{kDa}$

protein

Heat shock $70 \mathrm{kDa}$ protein

$1 \mathrm{~A} / \mathrm{B}$

Heat shock $70 \mathrm{kDa}$ protein 6

Heat shock protein HSP 90-

alpha

Heat shock protein HSP 90-beta

Heat shock protein beta-1

Heat shock-related $70 \mathrm{kD}$

protein 2

Heterogeneous nuclear

ribonucleoprotein Al

Heterogeneous nuclear

ribonucleoprotein $\mathrm{A} / \mathrm{B}$

Heterogeneous nuclear

ribonucleoproteins A2/B1

\section{isomerase}

Glucosidase 2 subunit beta

Glutathione S-transferase P

Glutathione S-transferase

omega-1

Glyceraldehyde-3-phosphate dehydrogenase

Guanine nucleotide-binding protein subunit beta-2-1

GTP-binding nuclear protein

\section{Ran}

Heat shock cognate $71 \mathrm{kDa}$

protein

Heat shock $70 \mathrm{kDa}$ protein

1A/1B

Heat shock-related $70 \mathrm{kDa}$

protein 2

Heat shock $70 \mathrm{kDa}$ protein 6

Heat shock protein HSP 90-

alpha

Heat shock protein HSP 90-

beta

Heat shock protein beta-1

Heterogeneous nuclear

ribonucleoprotein $\mathrm{A} 1$

Heterogeneous nuclear

ribonucleoprotein $\mathrm{A} / \mathrm{B}$

Heterogeneous nuclear

ribonucleoproteins A2/B1

Heterogeneous nuclear

ribonucleoprotein $\mathrm{A} 3$
Glucose-6-phosphate isomerase

Glucosidase 2 subunit beta

Glutathione S-transferase $P$

Glutathione S-transferase omega-1

Glyceraldehyde-3-phosphate dehydrogenase

Guanine nucleotide-binding protein subunit beta-2-1

GTP-binding nuclear protein Ran

Heat shock cognate $71 \mathrm{kDa}$ protein

Heat shock $70 \mathrm{kDa}$ protein $1 \mathrm{~A} / 1 \mathrm{~B}$

Heat shock-related $70 \mathrm{kDa}$ protein 2

政 $70 \mathrm{kDa}$ protein 6

Putative heat shock $70 \mathrm{kDa}$ protein 7

Heat shock protein HSP 90-alpha

Heat shock protein HSP 90-beta

Heat shock protein beta- 1

Heterogeneous nuclear ribonucleoprotein A1

Heterogeneous nuclear ribonucleoproteins A2/B1

Heterogeneous nuclear ribonucleoprotein A3 
Heterogeneous nuclear ribonucleoproteins $\mathrm{C} 1 / \mathrm{C} 2$ Heterogeneous nuclear ribonucleoprotein D0 Heterogeneous nuclear ribonucleoprotein $\mathrm{F}$

Heterogeneous nuclear ribonucleoprotein $\mathrm{G}$

Heterogeneous nuclear ribonucleoprotein $\mathrm{H}$

Heterogeneous nuclear ribonucleoprotein $\mathrm{H} 2$ Heterogeneous nuclear ribonucleoprotein $\mathrm{K}$ Heterogeneous nuclear ribonucleoprotein Q Heterogeneous nuclear ribonucleoprotein $\mathrm{R}$
Integrin beta- 1

Interleukin enhancer-binding factor 2

L-lactate dehydrogenase A chain

L-lactate dehydrogenase $B$ chain Lamin-A/C

Latexin
Heterogeneous nuclear ribonucleoproteins $\mathrm{C} 1 / \mathrm{C} 2$ Heterogeneous nuclear ribonucleoprotein D0 Heterogeneous nuclear ribonucleoprotein $\mathbf{F}$

Heterogeneous nuclear ribonucleoprotein $\mathrm{G}$

Heterogeneous nuclear ribonucleoprotein $\mathbf{H}$

Heterogeneous nuclear ribonucleoprotein $\mathrm{H} 2$

Heterogeneous nuclear ribonucleoprotein $\mathrm{K}$

Heterogeneous nuclear ribonucleoprotein $\mathrm{Q}$ Heterogeneous nuclear ribonucleoprotein $\mathrm{R}$

Histone $\mathrm{H} 4$

Importin subunit beta-1 Integrin beta-

Heterogeneous nuclear ribonucleoproteins $\mathrm{C} 1 / \mathrm{C} 2$ Heterogeneous nuclear ribonucleoprotein D0

Heterogeneous nuclear ribonucleoprotein $\mathrm{F}$

Heterogeneous nuclear ribonucleoprotein $\mathbf{H}$

Heterogeneous nuclear ribonucleoprotein $\mathrm{H} 2$

Heterogeneous nuclear ribonucleoprotein $\mathrm{K}$

Heterogeneous nuclear ribonucleoprotein Q

Heterogeneous nuclear ribonucleoprotein $\mathrm{R}$

Histone $\mathrm{H} 2 \mathrm{~A}$ type $1-\mathrm{B} / \mathrm{E}$

Histone $\mathrm{H} 2 \mathrm{~A}$ type 1-C

Histone H2A type 1-D

Histone $\mathrm{H} 2 \mathrm{~A}$ type $1-\mathrm{H}$

Histone $\mathrm{H} 2 \mathrm{~A}$ type $1-\mathrm{J}$

Histone $\mathrm{H} 4$

Importin subunit beta-1 Integrin beta-1

L-lactate dehydrogenase A chain

L-lactate dehydrogenase $\mathrm{B}$ chain Lamin-A/C

Latexin

chain

chain

Lamin-A/C

Latexin
Heterogeneous nuclear ribonucleoproteins $\mathrm{C} 1 / \mathrm{C} 2$

Heterogeneous nuclear ribonucleoprotein D0

Heterogeneous nuclear ribonucleoprotein $\mathrm{F}$

Heterogeneous nuclear ribonucleoprotein $\mathrm{H}$ Heterogeneous nuclear ribonucleoprotein $\mathrm{H} 2$

Heterogeneous nuclear ribonucleoprotein $\mathrm{K}$

Heterogeneous nuclear ribonucleoprotein Q

Heterogeneous nuclear ribonucleoprotein $\mathrm{R}$

Histone $\mathrm{H} 2 \mathrm{~A}$ type 1-B/E

Histone $\mathrm{H} 2 \mathrm{~A}$ type 1-C

Histone H2A type 1-D

Histone H2A type 1-H

Histone $\mathrm{H} 2 \mathrm{~A}$ type 1-J

Histone $\mathrm{H} 4$

Hypoxia up-regulated protein 1

Importin subunit beta-1

L-lactate dehydrogenase A

L-lactate dehydrogenase B
L-lactate dehydrogenase A chain

L-lactate dehydrogenase $B$ chain Lamin-A/C

Latexin 
Leucine-rich repeat-

containing protein 59

Microtubule-associated protein

Moesin

Myosin light polypeptide 6

Myosin regulatory light chain 12A

Myosin regulatory light chain 12B

Myosin regulatory light

polypeptide 9

Myristoylated alanine-rich C-

kinase substrate

Nascent polypeptide-associated

complex subunit alph

Nestin

Neuroblast differentiation-

associated protein AHNAK

Nuclease-sensitive element-binding

protein 1

Nucleolin

Nucleophosmin

Nucleoside diphosphate kinase A

Nucleoside diphosphate kinase B

POTE ankyrin domain family

member E

Peripherin

Peroxiredoxin-1
Moesin

Myosin-9

Myosin light polypeptide 6

Myosin light chain 6B

Myosin regulatory light chain

12A

Myosin regulatory light chain

12B

Myosin regulatory light

polypeptide 9

Nascent polypeptide-associated

complex subunit alph

Nestin

Neuroblast differentiation-

associated protein AHNAK

Nuclease-sensitive element-

binding protein 1

Nucleolin

Nucleophosmin

Nucleoside diphosphate kinase

A

Nucleoside diphosphate kinase

B

Nucleosome assembly protein

1-like 1

POTE ankyrin domain family

member $\mathrm{E}$

Peripherin

Peroxiredoxin-1
Lysosome-associated membrane glycoprotein

Microtubule-associated protein 4 Moesin

\section{Myosin light polypeptide 6}

Myosin light chain 6B

Myosin light chain 6B

Myosin regulatory light chain $12 \mathrm{~A}$

Myosin regulatory light

chain 12B

Myosin regulatory light

polypeptide 9

Myosin regulatory light chain $12 \mathrm{~A}$

Myosin regulatory light chain $12 \mathrm{~B}$

Myosin regulatory light polypeptide 9

Nascent polypeptide-associated complex subunit alph

\section{Nestin}

Neuroblast differentiationassociated protein AHNAK

Nucleolin

Nucleophosmin

Nucleoside diphosphate

kinase A

Nucleoside diphosphate kinase B

Nestin

Neuroblast differentiation-associated

protein AHNAK

Nuclease-sensitive element-binding protein

Nucleolin

Nucleophosmin

Nucleoside diphosphate kinase A

Nucleoside diphosphate kinase B

POTE ankyrin domain

family member $\mathrm{E}$

Peripherin

Peroxiredoxin-1
POTE ankyrin domain family member $\mathrm{E}$

Peroxiredoxin-1 
Peroxiredoxin-2

Peroxiredoxin-6

Peptidyl-prolyl cis-trans isomerase

Peptidyl-prolyl cis-trans isomerase

Phosphoglycerate kinase

Phosphoglycerate mutase 1

Plastin-3

Poly(rC)-binding protein

Poly(rC)-binding protein 2

Poly(rC)-binding protein 3

Polyadenylate-binding protein 1

Polyadenylate-binding protein 3 Polyadenylate-binding protein 4

Polypyrimidine tract-binding protein 1

Proactivator polypeptide

Profilin-1

Proliferation-associated protein

2G4

Prolyl 3-hydroxylase 1

\section{Protein SET}

Protein S100-A11

Protein disulfide-isomerase

Protein disulfide-isomerase A3

Protein disulfide-isomerase A4

Protein disulfide-isomerase A6

Pyruvate kinase isozymes M1/M2
Peroxiredoxin-2

Peroxiredoxin- 6

Peptidyl-prolyl cis-trans

isomerase $\mathrm{A}$

Peptidyl-prolyl cis-trans

isomerase $\mathbf{B}$

Phosphoglycerate kinase 1

Phosphoglycerate mutase 1

Plasminogen activator inhibitor

1 RNA-binding prot

Plastin-3

Poly(rC)-binding protein 1

Poly(rC)-binding protein 2

Poly(rC)-binding protein 3

Polyadenylate-binding protein I

Polyadenylate-binding protein 3

Polypyrimidine tract-binding protein 1

Proactivator polypeptide

Profilin-1

Prostaglandin E synthase 3

Protein S100-A11

Protein disulfide-isomerase

Protein disulfide-isomerase A3

Protein disulfide-isomerase A4

Protein disulfide-isomerase A6

Pyruvate kinase isozymes
Peroxiredoxin-2

Peroxiredoxin-6

Peptidyl-prolyl cis-trans

isomerase A

Peptidyl-prolyl cis-trans

isomerase B

Phosphoglycerate kinase 1

Phosphoglycerate mutase 1

Plastin-3

Plectin-1

Poly(rC)-binding protein 1

Poly(rC)-binding protein 2

Poly(rC)-binding protein 3

Polyadenylate-binding

protein 1

Polyadenylate-binding

protein 3

Proactivator polypeptide Profilin-1

Protein disulfide-isomerase Protein disulfide-isomerase

Protein disulfide-isomeras A6

Pyruvate kinase isozymes
Proactivator polypeptide Profilin-1

Protein S100-A11

Peroxiredoxin-2

Peroxiredoxin-6

Peptidyl-prolyl cis-trans isomerase A

Peptidyl-prolyl cis-trans isomerase B

Phosphoglycerate mutase 1

Poly(rC)-binding protein 1

Polyadenylate-binding protein 1

Polyadenylate-binding protein 3

Protein disulfide-isomerase

Protein disulfide-isomerase A3

Protein disulfide-isomerase A4

Protein disulfide-isomerase A6

Pyruvate kinase isozymes M1/M2 
Rab GDP dissociation inhibitor alpha

Rab GDP dissociation inhibitor

beta

Ras GTPase-activating-like protein

IQGAP1

Ras-related protein Rab-1A

Ras-related protein Rab-1B

Ras-related protein Rab-10

Ras-related protein Rab-15

Rho GDP-dissociation inhibitor

RNA-binding protein EWS

RNA-binding protein FUS

Radixin

Reticulon-4

Serine/threonine-protein

phosphatase PP1-alpha

Serine/threonine-protein

phosphatase PP1-beta

Serine/threonine-protein

phosphatase PP 1-gamma

Serpin H1

Small nuclear ribonucleoprotein

Sm D3

Spectrin alpha chain, brain

Spliceosome RNA helicase BAT1
Rab GDP dissociation inhibitor alpha

Rab GDP dissociation inhibitor

beta

Ras-related protein Rab-1A

Ras-related protein Rab-1B

Putative Ras-related protein

Rab-1C

Ras-related protein Rab-10

Ras-related protein Rab-15

RNA-binding protein EWS

RNA-binding protein FUS

Radixin

Reticulon-4

Serine/threonine-protein phosphatase PP 1-alpha Serine/threonine-protein phosphatase PP1-beta

Serine/threonine-protein phosphatase PP1-gamma

Serpin $\mathrm{H} 1$

Spectrin alpha chain, brain Spliceosome RNA helicase BAT1
$\mathrm{M} 1 / \mathrm{M} 2$

Rho GDP-dissociation

inhibitor 1

Rab GDP dissociation

inhibitor beta

Ras GTPase-activating-like

protein IQGAP1

Ras-related protein Rab-1A Ras-related protein Rab-1A

Ras-related protein Rab-1B Ras-related protein Rab-1B

Ras-related protein Rab-10

Ras-related protein Rab-15

Putative Ras-related protein Rab-1C

Ras-related protein $\mathrm{Rab}-10$

Ras-related protein Rab-15

RNA-binding protein EWS

RNA-binding protein FUS

Radixin

RNA-binding protein EWS

Radixin

Reticulocalbin-1

Reticulocalbin-3

Reticulon-4

Ribosome-binding protein 1

SH3 domain-binding glutamic acid-rich-

like protein

Serpin $\mathrm{Hl}$

Serpin $\mathbf{H} 1$

Spectrin alpha chain, brain 
Splicing factor $3 \mathrm{~B}$ subunit 2 Splicing factor U2AF $65 \mathrm{kDa}$ subunit

TATA-binding protein-associated factor $2 \mathrm{~N}$

T-complex protein 1 subunit beta T-complex protein 1 subunit gamma

T-complex protein 1 subunit delta T-complex protein 1 subunit epsilon

T-complex protein 1 subunit theta T-complex protein 1 subunit zeta

Talin-1

Thioredoxin domain-containing protein 5

Transgelin
Transgelin-2
Transitional endoplasmic reticulum
ATPase
Transketolase

Triosephosphate isomerase

Tropomyosin alpha-3 chain Tropomyosin alpha-4 chain
Splicing factor U2AF $65 \mathrm{kDa}$ subunit

TATA-binding proteinassociated factor $2 \mathrm{~N}$

T-complex protein 1 subunit gamma

T-complex protein 1 subunit delta

T-complex protein 1 subunit epsilon

T-complex protein 1 subunit theta

Talin-1

Thioredoxin

Thioredoxin domain-containing protein 5

Transaldolase

Transcription intermediary

factor 1-beta

Transgelin

Transgelin-2

Transitional endoplasmic

reticulum ATPase

Transketolase

Translationally-controlled tumor

protein

Triosephosphate isomerase

Tropomyosin alpha-1 chain

Tropomyosin alpha- 3 chain

Tropomyosin alpha-4 chain
Splicing factor 3B subunit 2

Splicing factor 3B subunit 2

TATA-binding protein

associated factor $2 \mathrm{~N}$

T-complex protein 1 subunit alpha

T-complex protein 1 subunit

beta

T-complex protein 1 subunit gamma

T-complex protein 1 subunit

delta

T-complex protein 1 subunit

epsilon

T-complex protein 1 subunit theta

Talin-1

Thioredoxin domaincontaining protein 5

Transgelin

Transgelin-2

Transitional endoplasmic

reticulum ATPase

Transketolase

Translationally-controlled

tumor protein

Triosephosphate isomerase

Tropomyosin alpha- 3 chain

Tropomyosin alpha-4 chain
T-complex protein 1 subunit alpha

T-complex protein 1 subunit beta

T-complex protein 1 subunit gamma

T-complex protein 1 subunit delta

T-complex protein 1 subunit epsilon

T-complex protein 1 subunit theta

T-complex protein 1 subunit eta

Talin-1

Thioredoxin domain-containing protein 5

Transgelin

Transgelin-2

Transitional endoplasmic reticulum ATPase Transketolase

Triosephosphate isomerase

Tropomyosin alpha-4 chain 
Tropomyosin beta chain

Tubulin alpha-1A chain

Tubulin alpha-1B chain

Tubulin alpha- $1 \mathrm{C}$ chain

Tubulin alpha-3C/D chain

Tubulin alpha-3E chain

Tubulin alpha-4A chain

Tubulin beta chain

Tubulin beta- 1 chain

Tubulin beta-2A chain

Tubulin beta-2B chain

Tubulin beta-2C chain

Tubulin beta- 3 chain

Tubulin beta- 4 chain

\section{UPF0568 protein C140rf166}

Ubiquitin

Ubiquitin carboxyl-terminal

hydrolase isozyme Ll

Ubiquitin-conjugating enzyme E2

variant 1

Ubiquitin-conjugating enzyme E2

variant 2

Vimentin

Vinculin

Voltage-dependent anion-selective

channel protein
Tropomyosin beta chain

Tubulin alpha-1 A chain

Tubulin alpha-1B chain

Tubulin alpha- $1 \mathrm{C}$ chain

Tubulin alpha-3C/D chain

Tubulin alpha-3E chain

Tubulin alpha-4A chain

Tubulin alpha- 8 chain

Tubulin beta chain

Tubulin beta- 1 chain

Tubulin beta-2A chain

Tubulin beta-2B chain

Tubulin beta-2C chain

Tubulin beta- 3 chain

Tubulin beta- 4 chain

Tubulin beta- 6 chain

UPF0568 protein C140rf166

\section{Ubiquitin}

Ubiquitin carboxyl-terminal

hydrolase isozyme $\mathbf{L} 1$

Ubiquitin-conjugating enzyme

E2 variant 1

Ubiquitin-conjugating enzyme

E2 variant 2

UV excision repair protein

RAD23 homolog $\mathrm{B}$

Vimentin

Vinculin
Tropomyosin beta chain

Tubulin alpha- $1 \mathrm{~A}$ chain

Tubulin alpha-1B chain

Tubulin alpha- $1 \mathrm{C}$ chain

Tubulin alpha-3C/D chain

Tubulin alpha-3E chain

Tubulin alpha-4A chain

Tubulin beta chain

Tubulin beta- 1 chain

Tubulin beta-2A chain

Tubulin beta-2B chain

Tubulin beta-2C chain

Tubulin beta- 3 chain

Tubulin beta- 4 chain

Tubulin beta- 6 chain

UPF0556 protein C19orf10

UPF0568 protein C14orf166 UPF0568 protein C14orf166

Ubiquitin

Ubiquitin-conjugating enzyme E2 variant 1

Ubiquitin-conjugating

enzyme E2 variant 2

UV excision repair protein

RAD23 homolog B

Vimentin
Ubiquitin

Tropomyosin beta chain

Tubulin alpha-1 A chain

Tubulin alpha-1B chain

Tubulin alpha- $1 \mathrm{C}$ chain

Tubulin alpha-3C/D chain

Tubulin alpha-3E chain

Tubulin alpha-4A chain

Tubulin alpha-8 chain

Tubulin beta chain

Tubulin beta-2A chain

Tubulin beta-2B chain

Tubulin beta-2C chain

Tubulin beta- 3 chain

Tubulin beta -4 chain

Tubulin beta- 6 chain

Ubiquitin-conjugating enzyme E2 variant 1

Vimentin 


\section{Table 3: PKG pull-down}

\section{Control \\ $60 \mathrm{kDa}$ heat shock protein, mitochondrial \\ 14-3-3 protein beta/alpha \\ 14-3-3 protein gamma \\ 14-3-3 protein zeta/delta \\ 14-3-3 protein epsilon \\ 14-3-3 protein eta \\ 14-3-3 protein sigma \\ 14-3-3 protein theta \\ $40 \mathrm{~S}$ ribosomal protein $\mathrm{SA}$ \\ 40S ribosomal protein $\mathrm{S} 3$ \\ $40 \mathrm{~S}$ ribosomal protein $\$ 5$ \\ $40 S$ ribosomal protein $\$ 9$ \\ $40 S$ ribosomal protein $S 18$ \\ $60 \mathrm{~S}$ ribosomal protein $\mathrm{L} 11$ \\ $60 S$ ribosomal protein L14}

$60 \mathrm{~S}$ acidic ribosomal protein $\mathrm{P0}$ $60 \mathrm{~S}$ acidic ribosomal protein $\mathrm{P} 2$

$78 \mathrm{kDa}$ glucose-regulated protein Acyl-CoA-binding protein

Actin, alpha cardiac muscle 1

Actin, alpha skeletal muscle

Actin, aortic smooth muscle

Actin, cytoplasmic 1

Actin, cytoplasmic 2

Actin, gamma-enteric smooth

muscle

Alpha-actinin-1

Alpha-actinin-4

Annexin A1

Annexin A2

Annexin A5
PE

$60 \mathrm{kDa}$ heat shock protein, mitochondrial

14-3-3 protein beta/alpha

14-3-3 protein gamma

14-3-3 protein zeta/delta

14-3-3 protein epsilon

14-3-3 protein eta

14-3-3 protein theta

40 S ribosomal protein $\mathrm{S3}$

$40 S$ ribosomal protein S5

$40 S$ ribosomal protein $S 9$

$40 S$ ribosomal protein $S 18$ Acyl-CoA-binding protein

Actin, alpha cardiac muscle 1

Actin, alpha skeletal muscle

Actin, aortic smooth muscle

Actin, cytoplasmic 1

Actin, cytoplasmic 2

Actin, gamma-enteric smooth

muscle

Annexin Al

Annexin A2

Annexin A5

\section{Cu}

$60 \mathrm{kDa}$ heat shock protein, mitochondrial

14-3-3 protein beta/alpha

14-3-3 protein gamma

14-3-3 protein zeta/delta

14-3-3 protein epsilon

$40 S$ ribosomal protein $S 3$

$40 S$ ribosomal protein $S 5$

$40 S$ ribosomal protein $S 18$

$78 \mathrm{kDa}$ glucose-regulated protein

Actin, alpha cardiac muscle 1

Actin, alpha skeletal muscle

Actin, aortic smooth muscle

Actin, cytoplasmic 1

Actin, cytoplasmic 2

Actin, gamma-enteric smooth

muscle

Annexin Al

Annexin A2

Annexin A5
$60 S$ ribosomal protein $\mathrm{L} 11$

60 S ribosomal protein L22

14-3-3 protein beta/alpha

14-3-3 protein gamma

14-3-3 protein zeta/delta

14-3-3 protein epsilon

14-3-3 protein eta

14-3-3 protein sigma

14-3-3 protein theta

$78 \mathrm{kDa}$ glucose-regulated protein

Actin, alpha cardiac muscle 1 Actin, alpha skeletal muscle Actin, aortic smooth muscle

Actin, cytoplasmic 1

Actin, cytoplasmic 2

Actin, gamma-enteric smooth muscle

Annexin A1

Annexin A2

Annexin A5 
Calmodulin

Calumenin

Chloride intracellular channel protein 1

\section{Cofilin-1}

Complement component $1 \mathrm{Q}$

subcomponent-binding prot

Cytochrome c

Elongation factor 1-alpha 1

Elongation factor 1-alpha 2

Elongation factor 2

Endoplasmin

Alpha-enolase

Beta-enolase

Gamma-enolase

Eukaryotic initiation factor 4A-I

Eukaryotic initiation factor 4A-II

Filamin-A

Fructose-bisphosphate aldolase A

Glucose-6-phosphate isomerase

Glyceraldehyde-3-phosphate dehydrogenase

GTP-binding nuclear protein Ran

Heat shock cognate $71 \mathrm{kDa}$ protein

Heat shock protein beta- 1
Calmodulin

Cathepsin B

Calmodulin

Cathepsin B

Cathepsin B

Clathrin heavy chain 1

Cofilin-1

Complement component $1 \mathrm{Q}$

subcomponent-binding prot

Elongation factor 1-alpha 1

Elongation factor 1-alpha 2

Elongation factor 2

Endoplasmin

Alpha-enolase

Beta-enolase

\section{Galectin-1}

Glucose-6-phosphate isomerase

Glutathione S-transferase P

Glyceraldehyde-3-phosphate dehydrogenase

GTP-binding nuclear protein Ran

Heat shock $70 \mathrm{kDa}$ protein $1 \mathrm{~A} / 1 \mathrm{~B}$

Heat shock $70 \mathrm{kDa}$ protein 6

Heat shock cognate $71 \mathrm{kDa}$

protein

\section{Cofilin-1}

Complement component $1 \mathrm{Q}$

subcomponent-binding prot

Cystatin-B

Elongation factor 1-alpha 1

Elongation factor 1-alpha 2

Elongation factor 2

\section{Alpha-enolase}

Beta-enolase

Gamma-enolase

Eukaryotic initiation factor 4A-I

Eukaryotic initiation factor 4A-I

Eukaryotic translation initiation

factor 5A-1

Eukaryotic translation initiation

\section{Galectin-1} dehydrogenase

GTP-binding nuclear protein Ran

Heat shock cognate $71 \mathrm{kDa}$ protein

Heat shock protein beta-1 factor $5 \mathrm{~A}-2$

\section{Glyceraldehyde-3-phosphate}

Cofilin-1

Elongation factor 1-alpha 1

Elongation factor 1-alpha 2

Elongation factor 2

Endoplasmin

Alpha-enolase

Beta-enolase

Gamma-enolase

Glucose-6-phosphate isomerase

Glyceraldehyde-3-phosphate dehydrogenase

GTP-binding nuclear protein Ran

Heat shock cognate $71 \mathrm{kDa}$ protein 
Heat shock protein HSP 90-alpha Heat shock protein HSP 90-beta

Hepatoma-derived growth factor

Heterogeneous nuclear ribonucleoprotein A1

Heterogeneous nuclear ribonucleoprotein $\mathrm{A} / \mathrm{B}$

Heterogeneous nuclear

ribonucleoproteins A2/B1

Heterogeneous nuclear

ribonucleoproteins $\mathrm{C} 1 / \mathrm{C} 2$

Heterogeneous nuclear

ribonucleoprotein D0

Heterogeneous nuclear ribonucleoprotein $H$

Heterogeneous nuclear

ribonucleoprotein $\mathrm{K}$

Histone $\mathrm{H} 4$

L-lactate dehydrogenase A chain

L-lactate dehydrogenase B chain

Moesin

Myosin light chain 6B

Myosin light polypeptide 6

Myristoylated alanine-rich C-kinase

substrate

Nucleolin

POTE ankyrin domain family

member E

Peptidyl-prolyl cis-trans isomerase

A

Peptidyl-prolyl cis-trans isomerase
Heat shock protein HSP 90-alpha Heat shock protein HSP 90-beta

Heterogeneous nuclear

ribonucleoproteins A2/B

Heterogeneous nuclear

ribonucleoproteins $\mathrm{C} 1 / \mathrm{C} 2$

Heterogeneous nuclear

ribonucleoprotein D0

Heterogeneous nuclear

ribonucleoprotein $\mathrm{K}$

Lamin-A/C

L-lactate dehydrogenase A

chain

L-lactate dehydrogenase $B$

chain

Myosin light chain 6B

Myosin light polypeptide 6

Myristoylated alanine-rich $\mathrm{C}$ -

kinase substrate

N-acetylglucosamine-6-

sulfatase

Nucleolar RNA helicase 2

Nucleolin

POTE ankyrin domain family

member $\mathrm{E}$

Peptidyl-prolyl cis-trans

isomerase $\mathrm{A}$

Peptidyl-prolyl cis-trans

isomerase $B$
Heat shock protein HSP 90 -alpha Heat shock protein HSP 90-beta

Heterogeneous nuclear

ribonucleoprotein A1

Heterogeneous nuclear

ribonucleoprotein $\mathrm{A} / \mathrm{B}$

Heterogeneous nuclear

ribonucleoproteins A2/B 1

Heterogeneous nuclear

ribonucleoproteins $\mathrm{C} 1 / \mathrm{C} 2$

Heterogeneous nuclear

ribonucleoprotein D0

\section{Histone $\mathrm{H} 4$}

L-lactate dehydrogenase A chain

L-lactate dehydrogenase B chain

Myosin light chain 6B

Myosin light polypeptide 6

Myristoylated alanine-rich C-kinase

substrate

Nucleolin

POTE ankyrin domain family

member E

Peptidyl-prolyl cis-trans isomerase

A

Peptidyl-prolyl cis-trans isomerase
Heat shock protein HSP 90-aipha Heat shock protein HSP 90-beta

Heterogeneous nuclear ribonucleoproteins $\mathrm{A} 2 / \mathrm{B} 1$

Heterogeneous nuclear ribonucleoprotein D0

\section{Lamin-A/C}

L-lactate dehydrogenase A chain

L-lactate dehydrogenase B chain

Myristoylated alanine-rich C-

kinase substrate

Nucleolin

Peptidyl-prolyl cis-trans isomerase 


\section{Peroxiredoxin-1}

Peroxiredoxin-2

Peroxiredoxin- 1

Phosphatidylethanolamine-binding

protein

Plasminogen activator inhibitor 1 RNA-binding prot

Poly(rC)-binding protein 1

Proactivator polypeptide

Profilin-1

Protein disulfide-isomerase

Protein disulfide-isomerase A3

Protein disulfide-isomerase A6

Pyruvate kinase isozymes M1/M2

RNA-binding protein EWS

RNA-binding protein FUS

Serpin H1

Splicing factor, arginine/serine-rich 3

TATA-binding protein-associated

factor $2 \mathrm{~N}$

$\mathrm{T}$-complex protein 1 subunit beta

Transgelin-2

Triosephosphate isomerase

Tubulin alpha-1 A chain

Tubulin alpha-1B chain

Tubulin alpha-1C chain

Tubulin alpha-3C/D

Tubulin alpha-3E chain

Tubulin alpha-4A chain

Tubulin beta chain
Profilin-1

Protein disulfide-isomerase

Protein disulfide-isomerase A3

Protein disulfide-isomerase A6

Pyruvate kinase isozymes

$\mathrm{M} 1 / \mathrm{M} 2$

Reticulocalbin-1

RNA-binding protein EWS

RNA-binding protein FUS

Serpin $\mathrm{Hl}$

TATA-binding proteinassociated factor $2 \mathrm{~N}$

Transgelin

Transgelin-2

Triosephosphate isomerase

Tubulin alpha-1A chain

Tubulin alpha-1B chain

Tubulin alpha- $1 \mathrm{C}$ chain

Tubulin alpha-3C/D

Tubulin alpha-3E chain

Tubulin beta chain
Peroxiredoxin-1

Peroxiredoxin-1

Proactivator polypeptide

Profilin-1

Protein disulfide-isomerase A3

Protein disulfide-isomerase A6

Pyruvate kinase isozymes M1/M2

Profilin-1

Protein disulfide-isomerase

Protein disulfide-isomerase A3

Protein disulfide-isomerase A6

Pyruvate kinase isozymes $\mathrm{M} 1 / \mathrm{M} 2$

RNA-binding protein EWS

RNA-binding protein FUS

SH3 domain-binding glutamic acid-

rich-like protein

Serpin $\mathbf{H} \mathbf{1}$

Serpin $\mathbf{H} 1$

TATA-binding protein-associated

factor $2 \mathrm{~N}$

Thioredoxin

Transgelin-2

Transgelin-2

Transitional endoplasmic reticulum ATPase

Triosephosphate isomerase

Tubulin alpha-1A chain

Tubulin alpha-1B chain

Tubulin alpha-1C chain

Tubulin alpha-3C/D

Tubulin alpha-3E chain

Tubulin alpha-4A chain

Tubulin beta chain
Tubulin alpha-1A chain

Tubulin alpha-1B chain

Tubulin alpha- $1 \mathrm{C}$ chain

Tubulin alpha-3C/D

Tubulin alpha-3E chain

Tubulin alpha-4A chain

Tubulin beta chain 
Tubulin beta-2A chain

Tubulin beta- $2 \mathrm{~B}$ chain

Tubulin beta- $2 \mathrm{C}$ chain

Tubulin beta- 3 chain

Tubulin beta- 4 chain

Tubulin-specific chaperone A

Transgelin

Transketolase

Ubiquitin

Ubiquitin carboxyl-terminal

hydrolase isozyme $\mathrm{L} 1$

Vimentin
Tubulin beta- $2 \mathrm{~A}$ chain

Tubulin beta-2B chain

Tubulin beta- $2 \mathrm{C}$ chain

Transketolase

Ubiquitin

Ubiquitin carboxyl-terminal

hydrolase isozyme L1

Vimentin
Tubulin beta- $2 \mathrm{~A}$ chain

Tubulin beta-2B chain

Tubulin beta-2C chain

Tubulin beta- 3 chain

Tubulin beta- 4 chain

Transgelin

Ubiquitin

Ubiquitin carboxyl-terminal

hydrolase isozyme $\mathrm{L}$

Vimentin
Tubulin beta- 1 chain

Tubulin beta-2A chain

Tubulin beta-2B chain

Tubulin beta-2C chain

Tubulin beta- 3 chain

\section{Transgelin}

Transketolase

Vimentin 


\section{Table 4: VEGFR-1 pull-down}

\section{Control}

PE

$10 \mathrm{kDa}$ heat shock protein

mitochondrial

$60 \mathrm{kDa}$ heat shock protein, $60 \mathrm{kDa}$ heat shock protein,

mitochondrial

14-3-3 protein beta/alpha

14-3-3 protein gamma

14-3-3 protein zeta/delta

14-3-3 protein eta

14-3-3 protein theta

14-3-3 protein epsilon

$40 \mathrm{~S}$ ribosomal protein $\mathrm{SA}$

40 S ribosomal protein $\$ 3$

$40 S$ ribosomal protein $\mathrm{S} 12$

40 S ribosomal protein $\mathrm{S} 18$

$60 S$ acidic ribosomal

protein $\mathrm{P} 2$

60S ribosomal protein $\mathrm{L1}$

$78 \mathrm{kDa}$ glucose-regulated protein mitochondrial

14-3-3 protein beta/alpha

14-3-3 protein gamma

14-3-3 protein zeta/delta

14-3-3 protein theta

14-3-3 protein epsilon

$40 \mathrm{~S}$ ribosomal protein $\mathrm{SA}$

$40 \mathrm{~S}$ ribosomal protein S3

$40 S$ ribosomal protein $S 18$

$60 \mathrm{~S}$ acidic ribosomal protein

$\mathrm{P} 2$

$60 S$ ribosomal protein $\mathrm{L} 7$

$78 \mathrm{kDa}$ glucose-regulated protein
$10 \mathrm{kDa}$ heat shock protein,

mitochondrial

14-3-3 protein beta/alpha

14-3-3 protein gamma

14-3-3 protein zeta/delta

14-3-3 protein theta

14-3-3 protein epsilon

$40 \mathrm{~S}$ ribosomal protein $\mathrm{SA}$

$40 \mathrm{~S}$ ribosomal protein $\mathrm{S} 3$

40S ribosomal protein $S 18$

$60 S$ ribosomal protein $\mathrm{L} 18$ $78 \mathrm{kDa}$ glucose-regulated protein
$40 \mathrm{~S}$ ribosomal protein $\mathrm{S} 18$

$60 S$ acidic ribosomal protein $\mathrm{P} 2$

60S ribosomal protein $\mathrm{L} 14$

$60 \mathrm{kDa}$ heat shock protein, mitochondrial

ATP synthase subunit alpha, mitochondrial

14-3-3 protein beta/alpha

14-3-3 protein gamma

14-3-3 protein zeta/delta

14-3-3 protein eta

14-3-3 protein theta

14-3-3 protein epsilon

14-3-3 protein sigma

$40 \mathrm{~S}$ ribosomal protein $\mathrm{SA}$

$40 S$ ribosomal protein $\$ 3$

40 S ribosomal protein $\$ 6$

$78 \mathrm{kDa}$ glucose-regulated protein 
Acyl-CoA-binding protein

Acyl-CoA-binding protein

ADP-ribosylation factor 1

ADP-ribosylation factor 3

ADP-ribosylation factor 4

ADP-ribosylation factor 5

Actin, alpha cardiac muscle

Actin, alpha skeletal

muscle

Actin, aortic smooth

muscle

Actin, gamma-enteric

smooth muscle

Actin, cytoplasmic 1

Actin, cytoplasmic 2

Adenylyl cyclase-

associated protein 1

Alpha-actinin-1

Alpha-actinin-4

Annexin A1

Annexin A2

Annexin A5

Brain acid soluble protein 1

Calmodulin

Calponin-2
Actin, alpha cardiac muscle 1

Actin, alpha skeletal muscle

Actin, aortic smooth muscle

Actin, gamma-enteric smooth

muscle

Actin, cytoplasmic 1

Actin, cytoplasmic 2

Alpha-actinin-1

Alpha-actinin-4

Annexin Al

Annexin A2

Annexin A5

Calmodulin

Calponin-2
Acyl-CoA-binding protein

ADP-ribosylation factor 1

ADP-ribosylation factor 3

ADP-ribosylation factor 4

ADP-ribosylation factor 5

ATP-dependent RNA helicase

DDX39

Actin, alpha cardiac muscle 1 Actin, alpha cardiac muscle 1

Actin, alpha skeletal muscle

Actin, alpha skeletal muscle

Actin, aortic smooth muscle

Actin, gamma-enteric

smooth muscle

Actin, aortic smooth muscle Actin, gamma-enteric smooth muscle

Actin, cytoplasmic 1

Actin, cytoplasmic 2

Adenylyl cyclase-associated protein 1

Alpha-actinin-1

Alpha-actinin-4

Annexin Al

Annexin A2

Annexin A5

Annexin A5

Astrocytic phosphoprotein

PEA-15

Calmodulin

Calmodulin

Calponin-2 


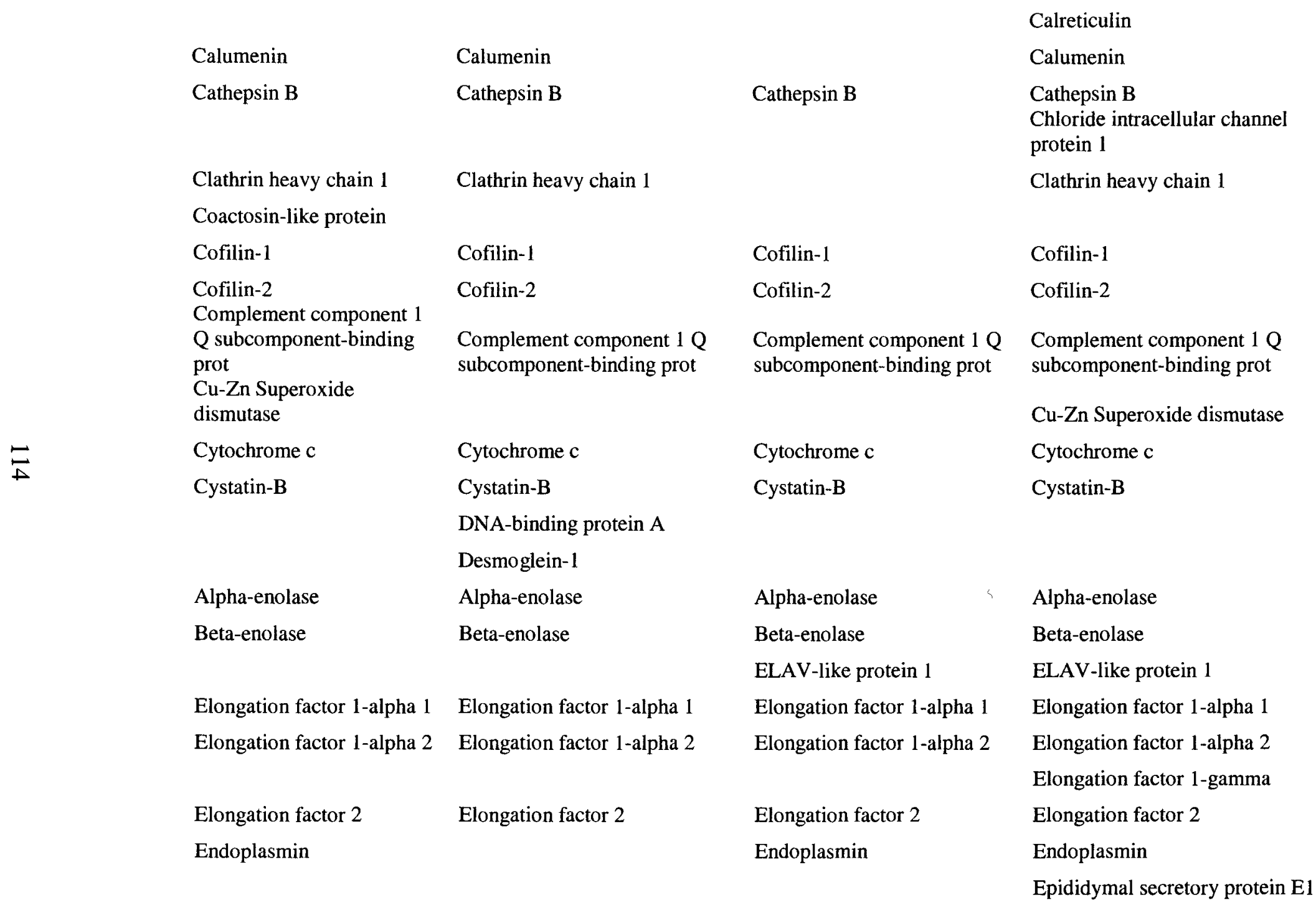


Eukaryotic initiation factor

4A-I

Eukaryotic initiation factor

4A-II

Eukaryotic translation

initiation factor 5A-1

Eukaryotic translation

initiation factor 5A-2

Far upstream element-

binding protein 1

Filamin-A

Filamin-B

Filamin-C

Fructose-bisphosphate

aldolase A

guclear

protein Ran

Galectin-1

Gamma-glutamyl

hydrolase

Glucosidase 2 subunit beta

Glyceraldehyde-3-

phosphate dehydrogenase

Guanine nucleotide-

binding protein subunit

beta-2-1

Heat shock protein beta-1

Heat shock $70 \mathrm{kDa}$ protein

$1 \mathrm{~A} / \mathrm{B}$

Heat shock $70 \mathrm{kDa}$ protein

Eukaryotic initiation factor 4A- Eukaryotic initiation factor I

Eukaryotic initiation factor 4A- Eukaryotic initiation factor II

Eukaryotic translation

initiation factor 5A-1

Eukaryotic translation

initiation factor $5 \mathrm{~A}-2$

Ezrin

Filamin-A

Filamin-B

Filamin-C

GTP-binding nuclear protein

Ran

Galectin-1

4A-II

Eukaryotic translation

initiation factor 5A-1

Eukaryotic translation

initiation factor 5A-2

Far upstream element-

binding protein 1

Filamin-A

$$
\text { aldolase } \mathrm{A}
$$

Fructose-bisphosphate

Galectin-1

Gamma-

glutamylcyclotransferase

Glyceraldehyde-3-phosphate

dehydrogenase

Heat shock protein beta-1

Heat shock $70 \mathrm{kDa}$ protein

$1 \mathrm{~A} / 1 \mathrm{~B}$

Heat shock $70 \mathrm{kDa}$ protein 6 dehydrogenase protein subunit beta- 2

Heat shock protein beta-1

Heat shock $70 \mathrm{kDa}$ protein

IA/1B
Eukaryotic initiation factor 4A-I

Eukaryotic initiation factor 4A-II

Eukaryotic translation initiation

factor 5A-1

Eukaryotic translation initiation

factor $5 \mathrm{~A}-2$

Ezrin

Far upstream element-binding

protein 1

Filamin-A

Fructose-bisphosphate aldolase

GTP-binding nuclear protein Ran

Glucosidase 2 subunit beta

Glutathione S-transferase P

Glyceraldehyde-3-phosphate

Glyceraldehyde-3-phosphate dehydrogenase

Guanine nucleotide-binding

Guanine nucleotide-binding protein subunit beta-2

Heat shock protein beta-1

Heat shock $70 \mathrm{kDa}$ protein

$1 \mathrm{~A} / \mathrm{B}$

Heat shock $70 \mathrm{kDa}$ protein 6 Heat shock $70 \mathrm{kDa}$ protein 6 
Heat shock cognate $71 \mathrm{kDa}$ protein

Heat shock protein HSP 90-alpha

Heat shock protein HSP

90-beta

Hepatoma-derived growth

factor

Heterogeneous nuclear ribonucleoprotein $\mathrm{A} / \mathrm{B}$

Heterogeneous nuclear ribonucleoprotein $\mathrm{A} 1$

Heterogeneous nuclear ribonucleoproteins A2/B

Heterogeneous nuclear ribonucleoproteins $\mathrm{Cl} / \mathrm{C} 2$

Heterogeneous nuclear ribonucleoprotein D0

Heterogeneous nuclear

ribonucleoprotein $\mathrm{F}$

Heterogeneous nuclear

ribonucleoprotein $\mathrm{G}$

Heterogeneous nuclear

ribonucleoprotein $\mathrm{H}$

Heterogeneous nuclear ribonucleoprotein $\mathrm{H} 3$

Heterogeneous nuclear ribonucleoprotein $\mathrm{K}$

High mobility group protein B 1

Histone $\mathrm{H} 2 \mathrm{~A}$ type 1-B/E

Histone H2A type 1-C

Histone H2A type 1-D
Heat shock cognate $71 \mathrm{kDa}$ protein

Heat shock protein HSP 90alpha

Heat shock protein HSP 90bet

beta

Hepatoma-derived growth

factor

Heterogeneous nuclear ribonucleoprotein A/B

Heterogeneous nuclear ribonucleoprotein $\mathrm{Al}$

Heterogeneous nuclear ribonucleoproteins $A 2 / B 1$

Heterogeneous nuclear ribonucleoproteins $\mathrm{C} 1 / \mathrm{C} 2$

Heterogeneous nuclear ribonucleoprotein D0

Heterogeneous nuclear

ribonucleoprotein $\mathrm{F}$

Heterogeneous nuclear

ribonucleoprotein $\mathrm{G}$

Heterogeneous nuclear

ribonucleoprotein $\mathrm{H}$

Heterogeneous nuclear ribonucleoprotein $\mathrm{H} 2$

Heterogeneous nuclear ribonucleoprotein $\mathrm{K}$ High mobility group protein B 1

ribonucleoprotein $\mathrm{A} / \mathrm{B}$

Heterogeneous nuclear

ribonucleoprotein Al

Heterogeneous nuclear

ribonucleoproteins A2/B

Heterogeneous nuclear

ribonucleoproteins $\mathrm{C} 1 / \mathrm{C} 2$

Heterogeneous nuclear

ribonucleoprotein D0

Heterogeneous nuclear

ribonucleoprotein $\mathbf{F}$

Heterogeneous nuclear

ribonucleoprotein $\mathrm{G}$

Heterogeneous nuclear

ribonucleoprotein $\mathrm{H}$

Heterogeneous nuclear

ribonucleoprotein $\mathrm{H} 2$

Heterogeneous nuclear

ribonucleoprotein $\mathrm{H} 3$

Heterogeneous nuclear

ribonucleoprotein $\mathrm{K}$

High mobility group protein

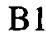

Heat shock cognate $71 \mathrm{kDa}$ protein

Heat shock protein HSP 90-alpha

Heat shock protein HSP 90-beta

Hepatoma-derived growth factor

Heterogeneous nuclear

ribonucleoprotein $\mathrm{A} / \mathrm{B}$
Heterogeneous nuclear

ribonucleoprotein A1

Heterogeneous nuclear

ribonucleoproteins A2/B1

Heterogeneous nuclear

ribonucleoproteins $\mathrm{C} 1 / \mathrm{C} 2$

Heterogeneous nuclear

ribonucleoprotein D0

Heterogeneous nuclear

ribonucleoprotein $\mathrm{F}$

Heterogeneous nuclear

ribonucleoprotein $\mathrm{G}$

Heterogeneous nuclear

ribonucleoprotein $\mathbf{H}$

Heterogeneous nuclear

ribonucleoprotein $\mathrm{H} 2$

Heterogeneous nuclear

ribonucleoprotein $\mathrm{H} 3$

Heterogeneous nuclear

High mobility group protein B1 ribonucleoprotein $\mathrm{K}$ 
Histone $\mathrm{H} 2 \mathrm{~A}$ type $1-\mathrm{H}$

Histone $\mathrm{H} 2 \mathrm{~A}$ type 1-J

Histone $\mathrm{H} 4$

Integrin beta-1

L-lactate dehydrogenase A chain

L-lactate dehydrogenase B

chain

Lamin-A/C

Lamina-associated

polypeptide 2 , isoform

alpha

Lamina-associated

polypeptide 2 , isoforms

beta/gam

Lysosome-associated

membrane glycoprotein 2

Microtubule-associated

protein 4

Moesin

Myosin light polypeptide 6

Myosin light chain 6B

Myosin regulatory light

polypeptide 9

Myosin regulatory light

chain 12A

Myosin regulatory light

chain 12B

Myristoylated alanine-rich

C-kinase substrate

\section{Histone $\mathrm{H} 4$}

Importin subunit beta-1

L-lactate dehydrogenase A chain

L-lactate dehydrogenase B chain

Lamin-A/C

Lysosome-associated
membrane glycoprotein 2

Microtubule-associated protein

IB

Moesin

Myosin light polypeptide 6

Myosin light chain 6B

Myosin regulatory light chain

12A

Myosin regulatory light chain

12B

Myristoylated alanine-rich Ckinase substrate
L-lactate dehydrogenase A

chain

L-lactate dehydrogenase B

chain

Lamin-A/C

Lamina-associated

polypeptide 2 , isoform alpha

Importin subunit beta-1

L-lactate dehydrogenase A chain

L-lactate dehydrogenase $B$ chain

Lamin-A/C

Lamina-associated polypeptide 2, isoform alpha

\section{Lamina-associated polypeptide 2, isoforms beta/gam}

Lysosome-associated membrane

glycoprotein 2

Microtubule-associated protein

IB

Microtubule-associated protein

4

Moesin

Myosin light polypeptide 6

Myosin light chain 6B

Myosin regulatory light chain

$12 \mathrm{~A}$

Myosin regulatory light chain

12B

Myristoylated alanine-rich C-

kinase substrate
Microtubule-associated protein 4

Moesin

Myosin light polypeptide 6

Myosin light chain 6B

Myosin regulatory light polypeptide 9

Myosin regulatory light chain $12 \mathrm{~A}$

Myosin regulatory light chain 12B

Myristoylated alanine-rich C-kinase substrate 
N-acetylglucosamine-6sulfatase

Nascent polypeptide-

associated complex subunit alph

Nestin complex subunit alph

Nuclease-sensitive elementbinding protein 1

Nucleolar RNA helicase 2

Nucleolin

Nucleophosmin

Nucleoside diphosphate kinase A

Nucleoside diphosphate

kinase B

Neuroblast differentiation-

POTE ankyrin domain

family member $\mathrm{E}$

Peptidyl-prolyl cis-trans

isomerase $\mathrm{A}$

Peptidyl-prolyl cis-trans

isomerase $B$

Peroxiredoxin-1

Peroxiredoxin-2

Peroxiredoxin-6

Phosphatidylethanolamine-

binding protein 1

Plasminogen activator

inhibitor 1 RNA-binding

prot

Poly(rC)-binding protein 1

\section{Nucleolin}

Nucleophosmin

Nucleoside diphosphate kinase

A

Nucleoside diphosphate kinase

B

Neuroblast differentiation-

associated protein AHNAK

POTE ankyrin domain family

member E

Peptidyl-prolyl cis-trans

isomerase A

Peptidyl-prolyl cis-trans

isomerase $\mathrm{B}$

Peroxiredoxin-1

Peroxiredoxin-2

Peroxiredoxin-6

Phosphatidylethanolamine-

binding protein 1

Poly(rC)-binding protein 1
$\mathrm{N}$-acetylglucosamine-6-sulfatase

Nascent polypeptide-associated complex subunit alph

Nascent polypeptide-associated complex subunit alph

Nucleolin

Nucleophosmin

Nucleophosmin

Nucleoside diphosphate kinase A

Nucleoside diphosphate kinase

B

Neuroblast differentiation-

associated protein AHNAK

POTE ankyrin domain family

member $\mathrm{E}$

Peptidyl-prolyl cis-trans

isomerase $\mathrm{A}$

Peptidyl-prolyl cis-trans

isomerase B

Peroxiredoxin-1

Peroxiredoxin-2

Peroxiredoxin-6

Phosphatidylethanolamine-

binding protein 1

Nucleoside diphosphate kinase A

Nucleoside diphosphate kinase B

Neuroblast differentiation-associated

protein AHNAK

POTE ankyrin domain family member $\mathrm{E}$

Peptidyl-prolyl cis-trans isomerase A

Peptidyl-prolyl cis-trans isomerase B

Peroxiredoxin-1

Peroxiredoxin-2

Peroxiredoxin-6

\section{Phosphoglycerate mutase 1}

Plasminogen activator inhibitor 1 RNAbinding prot

Poly(rC)-binding protein 1

Poly(rC)-binding protein 1 
Poly(rC)-binding protein $2 \quad$ Poly(rC)-binding protein 2

Poly(rC)-binding protein $3 \quad$ Poly(rC)-binding protein 3

Polyadenylate-binding Polyadenylate-binding protein

protein 1

Polyadenylate-binding

protein 3

Proactivator polypeptide

Profilin-1

Protein CDV3 homolog

Protein SET

Protein S100-A11

Protein disulfide-isomerase Protein disulfide-isomerase

A3

Protein disulfide-isomerase

A6

Pyruvate kinase isozymes M1/M2
1

Polyadenylate-binding protein 3

Proactivator polypeptide

Profilin-1

Protein disulfide-isomerase

Protein disulfide-isomerase A3

Protein disulfide-isomerase A6

Pyruvate kinase isozymes

M1/M2

Radixin

RNA-binding protein EWS

RNA-binding protein FUS

RNA-binding motif protein, $X$ -

linked-like-2
Polyadenylate-binding protein 1

Polyadenylate-binding protein 3

Proactivator polypeptide

Profilin-1

Protein CDV3 homolog

\section{Protein SET}

Protein disulfide-isomerase

Protein disulfide-isomerase A3

Protein disulfide-isomerase A6

Pyruvate kinase isozymes M1/M2

\section{RNA-binding protein EWS}

RNA-binding protein FUS

RNA-binding motif

protein, $X$-linked-like-2

Reticulocalbin-1

Reticulon-4

Reticulocalbin-1

Reticulon-4
Poly $(\mathrm{rC})$-binding protein 2

Poly $(\mathrm{rC})$-binding protein 3

Polyadenylate-binding protein 1

Polyadenylate-binding protein 3

Proactivator polypeptide

Profilin-1

\section{Protein SET}

Protein S100-A11

Protein disulfide-isomerase

Protein disulfide-isomerase A3

Protein disulfide-isomerase A6

Pyruvate kinase isozymes M1/M2

Radixin

Ras-related protein Rab-1B

Ras-related protein Rab-10

Ras-related protein Rab-15

Rab GDP dissociation inhibitor beta

RNA-binding protein EWS

RNA-binding protein FUS

RNA-binding motif protein, $X$-linked-

like-2 
SH3 domain-binding glutamic acid-rich-like protein

Serpin $H$

Splicing factor $3 \mathrm{~B}$ subunit

Splicing factor,

arginine/serine-rich 3

\section{Stathmin}

Stress-induced-

phosphoprotein 1

T-complex protein 1

subunit gamma

TATA-binding protein-

associated factor $2 \mathrm{~N}$

Thioredoxin

Thioredoxin reductase 1 ,

cytoplasmic

Transgelin

Transgelin-2

Transketolase

Triosephosphate isomerase

Tropomyosin alpha- 1 chain

Tropomyosin alpha- 3 chain
SH3 domain-binding glutamic acid-rich-like protein

Serpin $\mathbf{H} 1$

Serpin H1

Serpin $\mathrm{H} 1$

Serum albumin

Splicing factor,

arginine/serine-rich 3

Splicing factor, arginine/serine-rich 3

Spliceosome RNA helicase BAT 1

Stress-induced-phosphoprotein 1

T-complex protein 1 subunit gamma

TATA-binding proteinassociated factor $2 \mathrm{~N}$

TATA-binding proteinassociated factor $2 \mathrm{~N}$

Thioredoxin reductase 1 , cytoplasmic

cytoplasmic

Thioredoxin domain-

containing protein 5

Thioredoxin domain-

containing protein 17

Transgelin

Transgelin-2

Transketolase

Triosephosphate isomerase

Transitional endoplasmic

reticulum ATPase
Thioredoxin domain-

containing protein 17

Transgelin

Transgelin-2

Transketolase

Triosephosphate isomerase
Stress-induced-phosphoprotein 1

T-complex protein 1 subunit beta

TATA-binding protein-associated factor $2 \mathrm{~N}$

Thioredoxin reductase 1, cytoplasmic

Thioredoxin domain-containing protein

17

Transgelin

Transgelin-2

Transketolase

Triosephosphate isomerase

Transitional endoplasmic reticulum

ATPase

Tropomyosin alpha-3 chain 
Tropomyosin alpha- 4 chain

Tropomyosin beta chain

Tubulin alpha-1A chain

Tubulin alpha-1B chain

Tubulin alpha-1C chain

Tubulin alpha-3C/D chain

Tubulin alpha-3E chain

Tubulin alpha-4A chain

Tubulin beta chain

Tubulin beta-2A chain

Tubulin beta-2B chain

Tubulin beta- $2 \mathrm{C}$ chain

Tubulin beta- 3 chain

Tubulin beta- 4 chain

Tubulin beta- 6 chain

Tubulin-specific chaperone

\section{A}

Ubiquitin

Ubiquitin carboxyl-

terminal hydrolase isozyme

L1

Ubiquitin-conjugating

enzyme E2 variant 1
Tubulin alpha-1A chain

Tubulin alpha-1B chain

Tubulin alpha-1C chain

Tubulin alpha-3C/D chain

Tubulin alpha-3E chain

Tubulin alpha-4A chain

Tubulin alpha-8 chain

Tubulin beta chain

Tubulin beta- 1 chain

Tubulin beta-2A chain

Tubulin beta- $2 \mathbf{B}$ chain

Tubulin beta- $2 \mathrm{C}$ chain

Tubulin beta- 3 chain

Tubulin beta- 4 chain

Tubulin-specific chaperone A

Ubiquitin

Ubiquitin carboxyl-terminal hydrolase isozyme $\mathrm{Ll}$

Ubiquitin-conjugating enzyme

E2 variant 1
Tropomyosin alpha-4 chain

Tropomyosin alpha-4 chain

Tropomyosin beta chain

Tubulin alpha-1 A chain

Tubulin alpha-1B chain

Tubulin alpha-1C chain

Tubulin alpha-3C/D chain

Tubulin alpha-3E chain

Tubulin alpha-4A chain

Tubulin beta chain

Tubulin beta- $2 \mathrm{~A}$ chain

Tubulin beta-2B chain

Tubulin beta- $2 \mathrm{C}$ chain

Tubulin beta- 3 chain

Tubulin beta- 4 chain

Tubulin-specific chaperone A

Ubiquitin

Ubiquitin carboxyl-terminal hydrolase isozyme Ll
Tubulin alpha-1A chain

Tubulin alpha-1B chain

Tubulin alpha-1C chain

Tubulin alpha-3C/D chain

Tubulin alpha-3E chain

Tubulin alpha-4A chain

Tubulin alpha- 8 chain

Tubulin beta chain

Tubulin beta- 1 chain

Tubulin beta-2A chain

Tubulin beta-2B chain

Tubulin beta- $2 \mathrm{C}$ chain

Tubulin beta- 3 chain

Tubulin beta- 4 chain

Tubulin beta- 6 chain

Tubulin-specific chaperone A

Ubiquitin

Ubiquitin carboxyl-terminal hydrolase isozyme $\mathrm{L} 1$

Ubiquitin-conjugating enzyme E2

variant 1

Ubiquitin-conjugating enzyme E2

variant 2 


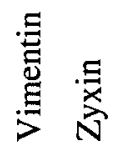

声

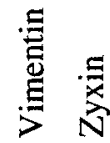

离 


\section{Vimentin Western Blot}

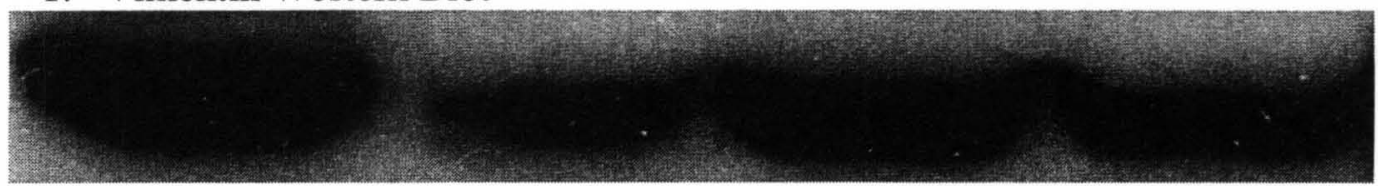

Control

PE

$\mathrm{Cu}$

$\mathrm{PE}+\mathrm{Cu}$

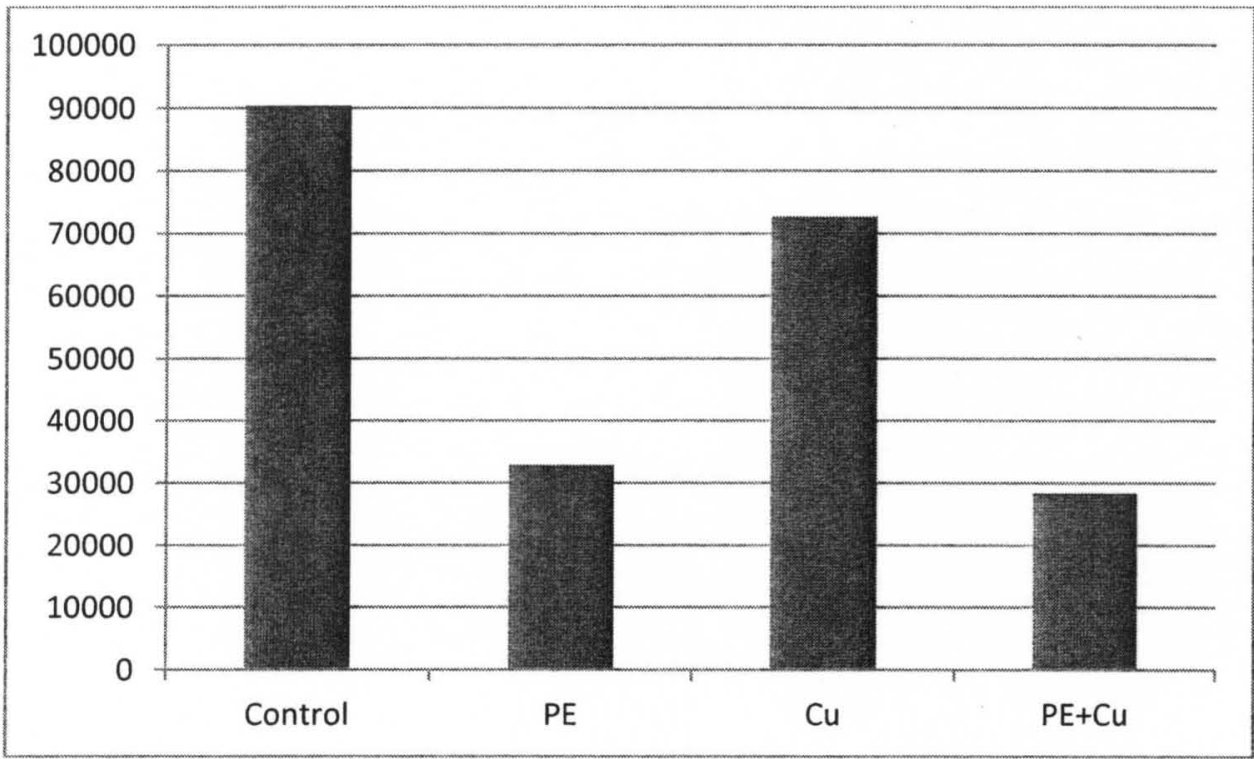

Figure 29. Representative Western blot of Vimentin under different treatment groups and densitometric analysis of Western blot. Immunoblotting was performed with anti-Vimentin antibody. This blot is representative of two independent experiments, and densitometric analysis is the mean of both blots. 
2. Interaction between Vimentin and PKG-1
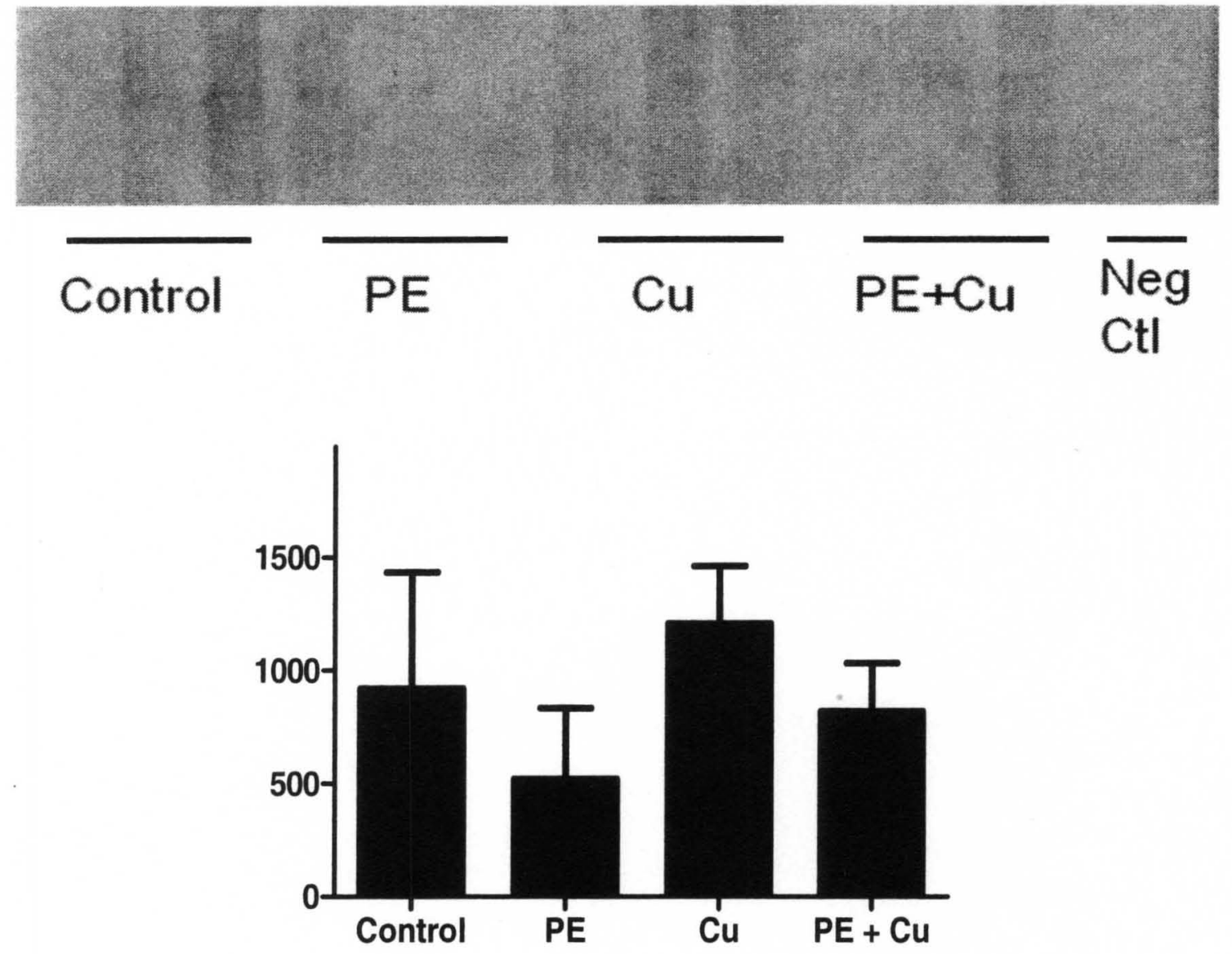

Figure 30. Co-immunoprecipitation of Vimentin and PKG-1. PKG-1 was coimmunoprecipitated after a Vimentin pull down. Semi-quantitative analysis of Western blot detecting PKG-1, bars are means for each group. One-way ANOVA, followed by Bonferroni's Multiple Comparison Test. No significant difference among groups. $n=3$. 
3. Interaction between Vimentin and VEGFR-1
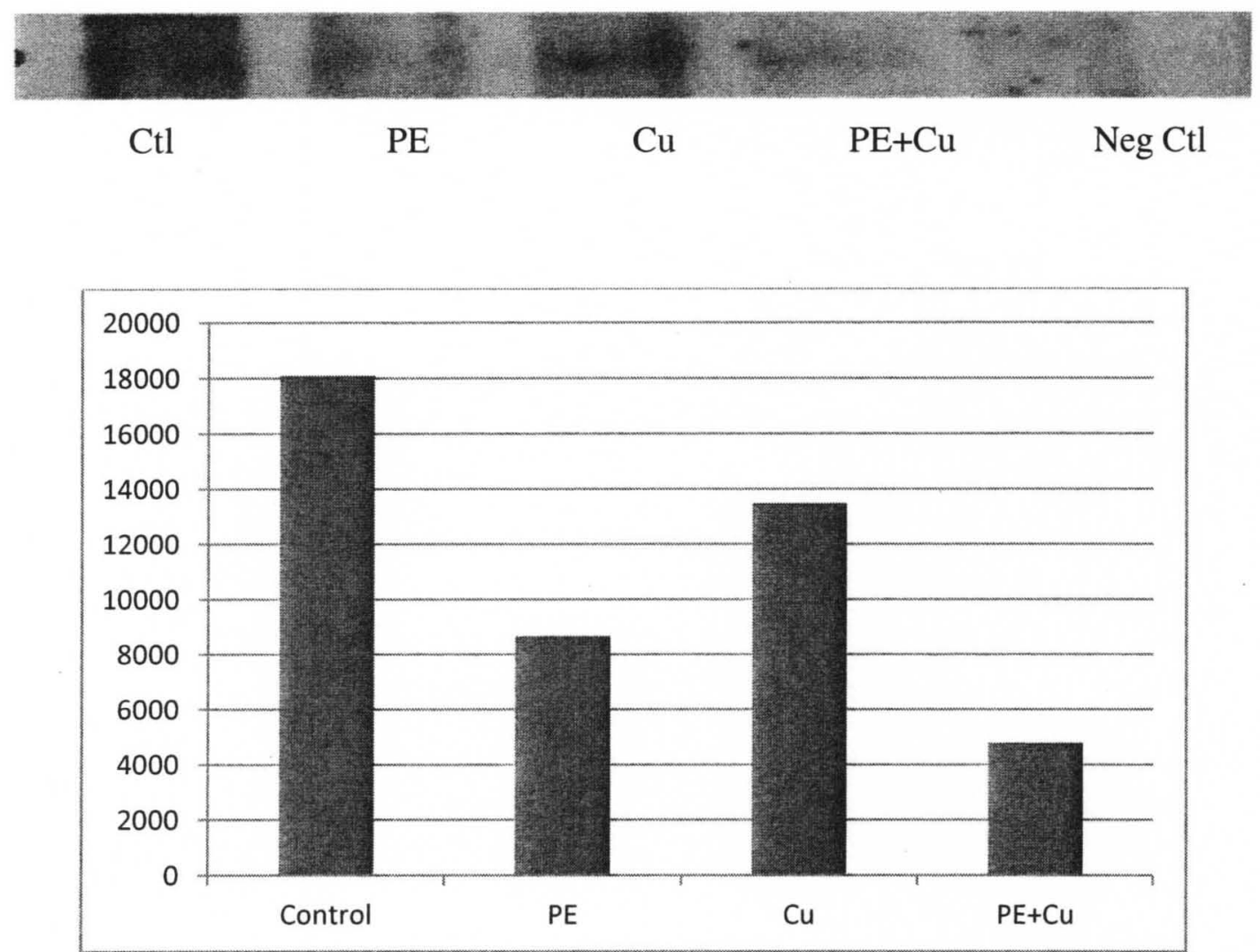

Figure 31. Co-immunoprecipitation of Vimentin and VEGFR-1. Semi-quantitative analysis of Western blot detecting VEGFR-1, bars are means from each group. $n=2$. 


\section{DISCUSSION}

It has been observed that $\mathrm{Cu}$ supplementation reverses cardiac myocyte hypertrophy of neonatal rat cardiac myocytes as well as embryonic cardiac myocytes derived from human embryos, and that this effect is VEGF-

dependent ${ }^{177}$. This study was undertaken to provide mechanistic insights into the action of $\mathrm{Cu}$. We have demonstrated that $\mathrm{Cu}$ at physiologically relevant levels which were used in this study does not increase VEGF production in primary cultures, yet anti-VEGF antibody blocked the reversal effect. This VEGFdependent action of $\mathrm{Cu}$ suggests that while $\mathrm{Cu}$ did not result in an increase in VEGF production, it did result in downstream events that are VEGF-dependent, and such downstream events would include VEGFRs. We observed that $\mathrm{Cu}$ increased the ratio of VEGFR-1:VEGFR-2, and that this increase was highly responsible for the reversal of cardiac myocyte hypertrophy (Figures 4 and 5).

This cause-effect relationship between the increase in the ratio of VEGFR-1 to VEGFR-2 and the reversal of cardiac myocyte hypertrophy was obtained from the experiment using siRNA targeting VEGFR-2 to elevate the ratio of VEGFR1:VEGFR-2. By using siRNA targeting VEGFR-2, we were able to increase the ratio of VEGFR-1:VEGFR-2 and thereby differentiate the regression phenomenon from the action of $\mathrm{Cu}$. However, it reproduced the same effect as $\mathrm{Cu}$-induced reversal of cardiac myocyte hypertrophy (Figures 9, 10 and 11). Further evidence 
from the experiment demonstrating that anti-VEGF antibody blocked the reversal of cardiac myocyte hypertrophy induced by VEGFR-2 gene silencing (Figures 12, 13 and 14), which is indicative of the importance of the interaction between VEGFR-1 and VEGF. siRNA targeting VEGFR-1 blocked Cu-induced reversal of cardiac myocyte hypertrophy, further indicating the role of VEGFR-1 in mediating $\mathrm{Cu}-$ induced reversal of cardiac myocyte hypertrophy (Figures 18, 19 and 20).

Numerous studies have established the differing functions of VEGFR-1 and VEGFR-2. VEGFR-2 is associated with cell growth stimulation by VEGF in endothelial cells. In cells with VEGFR-2 that are devoid of VEGFR-1, activation of VEGFR-2 by VEGF culminates in a mitogenic response ${ }^{178,179}$. Additionally, VEGFR-2 is more predominant in many of the known cellular responses elicited by VEGF such as embryonic vasculogenesis and tumor angiogenesis ${ }^{180}$. VEGFR-2 and its downstream pathways are more related to growth stimulation via MAPK signaling pathways. Akt1, which plays a critical role in the hypertrophic process has been linked to VEGFR-2, which was demonstrated in a study where a decoy VEGFR-2 inhibited cardiac growth induced by Akt1 activation ${ }^{181,182}$.

While previous studies have not given a clear, comprehensive understanding of the role of VEGFR-1 in cellular function, several studies have shown a counter action of VEGF-1 relative to VEGFR-2, and it was suggested that VEGFR-1 acted to negatively regulate VEGFR-2 ${ }^{183,184,185,186}$. This study demonstrates that VEGFR-1 is associated with PKG-1 activation. The link between VEGFR-1 to this well-known and well-studied pathway in the regression of heart hypertrophy provides an explanation of the VEGFR-1-dependent regression of cardiac myocyte hypertrophy. 
It is likely that many pathways are triggered by VEGFR-1 activation. Yet, PKG-1 signaling is the most studied and arguably the most important with respect to the regression of heart hypertrophy. We observed that siRNA targeting VEGFR-1 reduced PKG-1 activity (Figure 21), suggesting that the activation of PKG-1 is at least in part VEGFR-1-dependent. Conversely, the PKG-1 antagonist, Rp-8-pCPTcGMPS blocked both $\mathrm{Cu}$ and VEGFR-2 knockdown-induced reversal of cardiac myocyte hypertrophy (Figures 22-27). Taken together, these results demonstrate that VEGFR-1 is likely involved in $\mathrm{Cu}$-induced regression of cardiac myocyte hypertrophy, and that this pathway is likely associated with PKG-1 activation.

PKG-1 is activated in hypertrophic myocardium induced by pressure overload ${ }^{187}$. In mouse models of cardiac pressure overload induced by transverse aortic constriction (TAC), heart hypertrophy was observed 3 weeks after TAC, and PKG-1 activity was significantly elevated in the hypertrophic myocardium. Although PKG-1 activation alone was not sufficient to stop the development of heart hypertrophy, treatment with the oral phosphidiesterase-5A inhibitor, sildenafil, blocks the intrinsic catabolism of cGMP, leading to further activation of PKG-1 and resulted in the regression of heart hypertrophy. In our study, we observed that PE treatment enhanced PKG-1 activity, but Cu did not have any effect on PE-induced activation of PKG-1. But, $\mathrm{Cu}$ abrogated VEGFR-2, which is associated with Akt-1 signaling. In addition to this, inhibition was associated with the increase in the ratio of VEGF1:VEGFR-2, thus providing a molecular switch from growth via the VEGFR-2 pathway, to regression of cardiac myocyte hypertrophy via the VEGFR-1 pathway. While $\mathrm{Cu}$ did not increase PKG-1 activity, the switch in signaling pathways from 
VEGFR-2 to VEGFR-1 would make the activity of PKG-1 become dominant, leading to activation of hypertrophic reversal signaling cascades.

Initially, we hypothesized that the link between VEGFR-1 and PKG-1 was direct. However, after numerous VEGFR-1 and PKG-1 co-immunoprecipitations, we discovered that this was more than likely not the case. In order to elucidate the mechanism by which $\mathrm{Cu}$ increased VEGFR-1 signaling and thereby reversed cardiac myocyte hypertrophy, we turned to functional proteomics. Immune complex associated with VEGFR-1 or PKG-1 were sent off for analysis by liquid chromatography tandem mass spectrometry. Hundreds of proteins were identified. Upon further analysis of the data collected, we looked for proteins present in both the immune complexes of both VEGFR-1 and PKG-1. A literature search revealed very little knowledge regarding the role of Vimentin in the heart. Of the hundreds of proteins identified, we chose to further investigate the role of a protein interacting with both VEGFR-1 and PKG-1, Vimentin.

Preliminary results showed that PE decreases Vimentin in human cardiac myocytes. To validate the interaction between Vimentin and VEGFR-1 and Vimentin and PKG-1, co-immunoprecipitation was performed: pulling down Vimentin and analyzing the immunoprecipitate by Western blot to detect VEGFR-1, and then the reverse, pulling down VEGFR-1 and subjecting the immunoprecipiate to Western blot to detect Vimentin. The same experiment was performed to validate the proteinprotein interaction between PKG-1 and Vimentin.

Vimentin is the most abundant intermediate filament protein and is widely expressed. It is thought to have a primary role in biological structural processes, such 
as wound healing ${ }^{188}$ and is secreted by activated macrophages. Because of its abundance, it was assumed to play an important role in the stabilization of the cytoplasm, but knockout animals who do not express Vimentin have remarkably normal phenotypes ${ }^{189},{ }^{190}$. However, Vimentin knockout mice exhibited phenotypic abnormalities under specific conditions, such as injury ${ }^{191}$. Therefore, Vimentin may play a specialized role that contributes to distinct dynamic cellular processes ${ }^{192}$.

Previously thought to be an exclusively intracellular protein, recent studies have shown that Vimentin is also secreted by human macrophages into the extracellular space in a process that is regulated by pro- and anti-inflammatory cytokines ${ }^{193}$. Vimentin is phosphorylated at serine and threonine residues, and is phosphorylated by numerous protein kinases and is a substrate for protein kinase $\mathrm{C}$ (PKC). Additionally, the secretion of Vimentin is blocked by G06983, a specific PKC inhibitor. These findings are consistent with previous research that phosphorylation of Vimentin impacts its intracellular localization ${ }^{194},{ }^{195}$. Interestingly, PKC is also an essential mediator of heart hypertrophy ${ }^{196,197,198,199}$. Thus providing a possible link and further clue to the mechanism by which $\mathrm{Cu}$ can reverse cardiac hypertrophy. While the exact implications have yet to be understood, it is also interesting to note that Vimentin is also a known copper binding protein. It may also play a role in tissue repair ${ }^{157}$. We hypothesize that Vimentin acts as a molecular switch, somehow switching pathways from growth to regression upon the addition of $\mathrm{Cu}$.

Recently, a lot of attention has been given to the role of VEGF and it's receptors in cancer. In 2004, the FDA approved bevacizumab (Avastin ${ }^{\mathrm{TM}}$ ), the first approved biological therapy for the prevention of angiogenesis in tumors. It is one of many 
inhibitors developed on the idea of inhibiting angiogenesis in tumors and thereby delaying or completely halting the progression of cancer. Additionally, they have the benefit of fewer side effects than chemotherapy. However, results of these inhibitors may be dependent upon the type of cancer being treated. The investigators in a recent study that involved 2385 glioblastoma patients treated with small molecule kinase inhibitors suggested that the efficacy did not warrant their use in clinical practice ${ }^{200}$. Other studies have shown that the prognostic value of VEGF and Flt-1 expression in tumors has yet to be determined, and in patients with stage II/III non-small cell lung cancer, tumor expression did not appear to have a significant impact on overall survival, locoregional control or metastases-free survival ${ }^{201}$. While there is great potential for drugs targeting VEGF and its receptors, there is clearly also a need to proceed with caution and take into careful consideration if the potential cardiovascular side effects outweigh the risk.

There were several limits to this study. We did not examine how $\mathrm{Cu}$ regulates the expression or differentialdistribution of VEGFRs. This is an important question and the answer to this question will help further understand the role of $\mathrm{Cu}$ in regression of heart hypertrophy. From our observation that $\mathrm{Cu}$ suppressed PE-elevated VEGFR-2 levels, it would suggest that $\mathrm{Cu}$ would not cause overexpression of VEGFR-1. Then the question needs to be addressed is how $\mathrm{Cu}$ reduces VEGFR-2, suppressing its expression or enhancing its degradation? Our future studies will address this question. Another limitation of this study is that the mechanism by which VEGFR-1 regulates PKG-1 activation has not been defined. While it is clear that Vimentin plays a role, the mechanistic insight is important for further understanding the signalling pathway 
leading to regression of cardiomyocyte hypertrophy, which will be an important focus in our future studies.

In conclusion, the present study demonstrates that $\mathrm{Cu}$-induced regression of cardiomyocyte hypertrophy is related to a switch of signaling pathways from VEGFR-2 to VEGFR-1. Although both pathways are VEGF-dependent, the data suggest that VEGFR-1 is associated with signaling pathways linked to PKG-1, which has been demonstrated both in vivo and in vitro to be associated with cardiomyocyte growth inhibition and regression of cardiomyocyte hypertrophy. It remains to be elucidative how $\mathrm{Cu}$ regulates the differential distribution between VEGFR-1 and VEGFR-2 in hypertrophic cardiomyocytes and how VEGFR-1 interacts with PKG-1 leading to the activation of hypertrophic reversal signalling pathways, however, we have observed that Vimentin is associated with both VEGFR-1 and PKG-1 suggesting it may act as a link between the two, or perhaps acts as a molecular switch between hypertrophic and reversal of regression signaling. Further studies using siRNA targeting Vimentin will be instrumental in testing the hypothesis that it is a critical component in the reversal effect. 


\section{REFERENCES}

1. Heart disease and stroke statistics: 2008 update. Dallax, TX: American Heart Association; 2008.

2. Keehan S. et al. National Health Spending Projections Through 2020: Economic Recovery and Reform Drive Faster Spending Growth, Health Affairs, 2011;30(8):1-12.

3. Chen, J. and Fishman, M. Zebrafish tinman homolog demarcates the heart field and initiates myocardial differentiation. Development, 1996;122: 3809-3816.

4. Grossman, W. Cardiac hypertrophy: Useful adaptation or pathological process? American Journal of Medicine, 1980;69(4):576-584.

5. Dorn, G. and Force T. Protein kinase cascade in the regulation of cardiac hypertrophy. J ournal of Clinical Investigation, 2005;115(3):527-537.

6. Frey N. and Olson EN. Cardiac hypertrophy: the good, the bad and the ugly. Annual Review of Physiology, 2003; 65:45-79.

7. Stewart S. et al. More 'malignant' than cancer? Five-year survival following a first admission for heart failure. European Journal of Heart Failure, 2001; 3(3): 315-322.

8. Valentine $\mathbf{J}$ and Gralla E. Delivering Copper Inside Yeast and Human Cells. Science, 1997;278:817-818.

9. Evans GW. Copper homeostasis in the mammalian system. Pathological Reviews, 1973;53:535-570.

10. Lowe, N. et al. Is there a potential therapeutic value of copper and zinc for osteoporosis? Proceedings of the Nutrition Society, 2002; 61:181-485.

11. Olivares, $M$ and Uauy R. Copper as an essential nutrient. American Journal of Clinical Nutrition, 1996;63: 791S-796S.

12. Folkman J and Shing Y. Angiogenesis, The Journal of Biological Chemistry, 1992;267:10931-10934.

13. Stemmer KL et al. Copper deficiency effects on cardiovascular system and lipid 
of the Society for Experimental Biology and Medicine, 1989;192:166-171.

15. Xie $\mathrm{H}$ and Kang YJ. Role of copper in angiogenesis and its medicinal implications. Current Medicinal Chemistry. 2009;16:1304-1314.

16. Berger, Rudolph et al. B-Type Natriuretic Peptide Predicts Sudden Death in Patients with Chronic Heart Failure, Circulation, 2002;105:2392-2397.

17. Mann, D.L. Mechanisms and Models in Heart Failure. Circulation, 1999;100: 999-1008.

18. Scarabelli, T.M., Knight, R., Stephanou, A., Townsend, P.A., Chen-Scarabelli, C., Lawrence, K., Gottlieb, R., Latchman, D., and Narula, J. Clinical implications of apoptosis in ischemic myocardium. Current Problems in Cardiology, 2006; 31:181-264.

19. Apstein CA, morgan JP: Cellular mechanisms underlying left ventricular diastolic function. Pp. 3-24. In Gaasch WH, Le Winter MM (eds): Left ventricular diastolic dysfunction and Heart Failure. Lea \& Febiger, Philadelphia, 1994.

20. Morgan JP: Mechanisms of disease. Abnormal intracellular modulation of calcium as a major cause of cardiac contractile dysfunction..New England Journal of Medicine, 1991;325:625-632.

21. Katz AM: Interplay between inotropic and lusitropic effects of cyclic adenosine monophosphate on the myocardial cell. Circulation 1990;82:7-11.

22. Rich, M.W., Nease, R.F. Cost-Effective Analysis in Clinical Practice The Cost of Heart Failure. Archives of Internal Medicine. 1999; 159:1690-1700.

23. American Heart Association. Heart Disease and Stroke Facts, 2006 Update. Dallas, Texas: AHA, 2006.

24. Dipla, Konstantina et al. Myocyte Recovery After Mechanical Circulatory Support in Humans With End-Stage Heart Failure. Circulation. 1998;97:23162322.

25. Lopez AD. Assessing the burden of mortality from cardiovascular diseases. World Health Statatistics Quarterly, 1993;46(2):91-6.

26. Pearson TA. Cardiovascular disease in developing countries: myths, realities, and opportunities. Cardiovascular Drugs and Therapy, 1999;13(2):95-104.

27. Wielgosz AT. The decline in cardiovascular health in developing countries. World Health Statistics Quarterly, 1993;46(2):90. 
28. Feenstra, Johannes et al. Drug-Induced Heart Failure, Journal of the American College of Cardiology, 1999;33(5):1152-62.

29. Turnlund JR. Copper. In: Shils ME, Shike M, Ross AC, Caballero B, Cousins RJ, eds. Modern Nutrition in Health and Disease. 10th ed. Philadelphia: Lippincott Williams \& Wilkins; 2006:289-299.

30. Zatuchni, G. I. Advances in Contraception. Advances in Contraception, 1989;5(4):193-196.

31. The historical part of this paper is based on H.H.A. Dollwet and J.R.J. Sorenson, Historic uses of copper compounds in medicine, Trace Elements in Medicine, 1985;2(2):80-87.

32. Linder, M.C. (1991). Biochemistry of Copper (New York: Plenum Press).

33. Pen a, M.M.O., Lee, J., and Thiele, D.J. A delicate balance: Homeostatic control of copper uptake and distribution. Journal of Nutrition, 1999:129(7): 1251-1260.

34. Puig, S., and Thiele, D.J. Molecular mechanisms of copper uptake and distribution. Current Opinion in Chemical Biology, 2002:6(2):171-180.

35. Wester PO. Trace elements in human myocardial infarction determined by neutron activation analysis. Acta Medica Scandanavia, 1965:178(6):765-788.

36. Schroeder, H.A. et al. Essential trace metals in man: copper. Journal of Chronic Diseases, 1996:19(9):1007-1034.

37. Taylor, G O \& Wiilliams AO. Lipid and trace metal content in coronary arteries of Nigerian Africans. Experimental Molecular Pathology 1974:21(3):371-380.

38. Anderson TW et al. Letter:ischemic heart disease, water hardness and myocardial magnesium. Canadian Medical Association Journal, 1975: 113(3):199-203.

39. Chipperfield B \& Chipperfield JR. Differences in metal content of the heart muscle in death from ischemic heart disease. American Heart Journal, 1995: 95(6):732-737.

40. Casey CE, Guthrie BE \& Robinson MF. Copper, manganese, zinc and cadmium in tissues from New Zealanders. Biology of Trace Elemental Research, 1982:4105-4115.

41. Reiser S, et al. Indices of copper status in humans consuming a typical 
American diet containing either fructose or starch. American Journal of Clinical Nutrition, 1985:42(2):242-251.

42. Zama N, Towns RLR. Cardiac copper, magnesium, and zinc in recent and old myocardial infarction. Biology Trace Element Research 1986:19:201-208.

43. Martin BJ, Lyon TD \& Fell GS (1991). Comparison of inorganic elements from autopsy tissue of young and elderly subjects. J Trace Elem Electrolytes Health Dis 1991:5(3):203-211.

44. Falcone JC, Saari JT, Kang YJ \& Schuschke DA. Vasoreactivity in an adult rat model of marginal copper deficiency. Nutritional Research, 2005:25(2):25177-25186.

45. Nose Y, Kim B, Thiele E. Ctrl drives intestinal absorption and is essential for growth, iron metabolism, and neonatal cardiac function. Cell Metabolism, 2006:4(3):235-244.

46. Cherukuri S. et al. Unexpected role of ceruloplasmin in intestinal iron absorption. Cell Metabolism, 2005:2(5):309-319.

47. Schuschke, D. et al. Microvascular control mechanisms in copper deficiency. The Journal of Trace Elements in Experimental Medicine. 1996:9:63-72.

48. Jiang $Y$ et al. Dietary copper supplementation reverses hypertrophic cardiomyopathy induced by chronic pressure overload in mice. Journal of Experimental Medicine. 2007:204 (3):657-666.

49. N. Rusinko and J.R. Prohaska. Adenine nucleotide and lactate levels in organs from copper-deficient mice and brindled mice. Journal of Nutrition. 1985:115(7):936-943.

50. J.C.J. Chao, D.M. Medeiros, R.A. Altschuld and C.M. Hohl. Cardiac nucleotide levels and mitochondrial respiration in copper-deficient rats. Comparative Biochemistry and Physiology. 1993:104(1):163-168.

51. A.M. Bode, L.A. Miller, J. Faber and J.T. Saari, Mitochondrial respiration in heart, liver and kidney of copper-deficient rats, Journal of Nutritional Biochemistry. 1992:3(12): 668-672.

52. J.M. Matz, J.T Saari and A.M. Bode. Functional aspects of oxidative phosphorylation and electron transport in cardiac mitochondria of copperdeficient rats. Journal of Nutritional Biochemistry. 1995:6(12):644-652.

53. Rae TD et al. Undetectable Intracellular Free Copper: The Requirement of a Copper Chaperone for Superoxide Dismutase. Science. 1999:284(5415):805-808. 
54. Hart EB, Steenbock J, Waddell J, Elvehjem CA. Iron in nutrition. VII. Copper as a supplement to iron for hemoglobin building in the rat. Journal of Biological Chemistry. 2002;277(34)e22.

55. Chou T, Adolph WH. Copper metabolism in man. Biochemistry Journal. 1935;29(2):476-9.

56. Tompsett SL. The copper and "inorganic" iron contents of human tissues. Biochemical Journal. 1935;29(2):480-6.

57. Tompsett SL. The excretion of copper in urine and faeces and its relation to the copper content of the diet. Biochemical Journal. 1934;28(6):2088-91.

58. Leverton RM, Binkley ES. The copper metabolism and requirement of young women. Journal of Nutrition. 1944;27(1):43-52.

59. Food and Nutrition Board, Institute of Medicine. Copper. Dietary reference intakes for vitamin $\mathrm{A}$, vitamin $\mathrm{K}$, boron, chromium, copper, iodine, iron, manganese, molybdenum, nickel, silicon, vanadium, and zinc. Washington, D.C.: National Academy Press; 2001:224-257

60. Kim BE, Nevitt T \& Thiele DJ . Mechanisms for copper acquisition, distribution and regulation. Nature Chemical Biology. 2008:4(3): 176-185.

61. Hellman NE \& Gitlin JD. Ceruloplasmin metabolism and function. Annual Reviews of Nutrition. 2002:22439-22448.

62. Turnland J. Human whole-body copper metabolism. American Journal of Clinical Nutrition. 1998:67:960-4S.

63. Turnland JR, Keen CL, Smith RG. Copper status and urinary and salivary copper in young men at three levels of dietary copper. American Journal of Clinical Nutrition. 1990;51:658-64.

64. Milne DB. Copper intake and assessment of copper status. American Journal of Clinical Nutrition. 1998:67(5):1041S-1045S.

65. Konig JS \& Elmadfa I. Plasma copper concentration as marker of copper intake from food. Annals of Nutrition and Metababolism. 2000:44(3):129134.

66. Araya M, Olivares M, Pizarro F, Gonzalez M, Speisky H \& Uauy R. Copper exposure and potential biomarkers of copper metabolism. Biometals. 2003:16(1):199-204. 
67. Kanikowska D, Grzymislawski M \& Wiktorowicz K. Seasonal rhythms of “acute phase proteins" in humans. Chronobiology International. 2005:22(3): 591-596.

68. Montagna O, Grosso R, Santoro A \& Mautone A. Plasma levels of the serum antioxidants (uric acid, ceruloplasmin, transferrin) in term and preterm neonates in the first week of life. Minerva Pediatrica. 1994:46(6): 255-260.

69. Hambidge M. Biomarkers of trace mineral intake and status. Journal of Nutrition. 2003:133:Suppl. 3:948S-955S.

70. Bertinato J, Iskandar M \& L'Abbe MR. Copper deficiency induces the upregulation of the copper chaperone for $\mathrm{Cu} / \mathrm{Zn}$ superoxide dismutase in weanling male rats. Journal of Nutrition. 2003:133(1):28-31.

71. Ferrara N, Henzel WJ. Pituitary follicular cells secrete a novel heparin-binding growth factor specific for vascular endothelial cells. Biochemical and Biophysical ResearchCommunications. 1989:161(2):851-858.

72. Leung DW, Cachianes G, Kuang W-J, Goeddel DV, Ferrara N. Vascular endothelial growth factor is a secreted angiogenic mitogen. Science. 1989:246:1306-1309.

73. Connolly DT, Heuvelman DM, Nelson R, Olander JV, Eppley BL, Delfino JJ, Siegel NR, Leimgruber RM, Feder J. Tumor vascular permeability factor stimulates endothelial cell growth and angiogenesis. Journal of Clinical Investigation. 1989:84(5):1470-1478.

74. Keck PJ, Hauser SD, Krivi G, Sanzo K, Warren T, Feder J, Connolly DT. Vascular permeability factor, an endothelial cell mitogen related to platelet derived growth factor. Science. 1989:246(4935):1309-1312.

75. Ploüet J, Schilling J, Gospodarowicz D. Isolation and characterization of a newly identified endothelial cell mitogen produced by AtT 20 cells. European Mecular Biology Organization Journal. 1989:8(12):3801-3806.

76. Conn G, Bayne M, Soderman L, Kwok PW, Sullivan KA, Palisi TM, Hope DA, Thomas KA. Amino acid and cDNA sequence of a vascular endothelial cell mitogen homologous to platelet-derived growth factor. Proceedings of the National Acadamy of Sciences USA 1990:87:2628-2632.

77. Pepper MS, Wasi S, Ferrara N, Orci L, Montesano R. In vitro angiogenic and proteolytic properties of bovine lymphatic endothelial cells. Experimental Cell Research.1994:210(2):298-305.

78. Senger DR, Galli SJ, Dvorak AM, Perruzzi CA, Harvey VS, Dvorak HF. Tumor 
cells secrete a vascular permeability factor that promotes accumulation of ascites fluid. Science. 1983:219(4587):983-985.

79. Connolly DT, Olander JV, Heuvelman D, Nelson R, Monsell R, Siegel N, Haymore BL, Leimgruber R, Feder J. Human vascular permeability factor. Isolation from U937 cells. Journal of Biological Chemistry. 1989:264(33):20017-20024.

80. Pekala P, Marlow M, Heuvelman D, Connolly D. Regulation of hexose transport in aortic endothelial cells by vascular permeability factor and tumor necrosis factor-alpha, but not by insulin. Journal of Biological Chemistry. 1990:265(30):18051-18054.

81. Melder RJ, Koenig GC, Witwer BP, Safabakhsh N, Munn LL, Jain RK. During angiogenesis, vascular endothelial growth factor and basic fibroblast growth factor regulate natural killer cell adhesion to tumor endothelium. Nature Medicine. 1996:2(9):992-997.

82. Kim I, et al. VEGF Stimulates Expression of ICAM-1, VCAM-1 and E-Selectin through Nuclear Factor- $\kappa$ B Activation in Endothelial Cells. Journal of Biological Chemistry. 2006:276:7614-7620.

83. Clauss M, Gerlach M, Gerlach H, Brett J, Wang F, Familletti PC, Pan Y-C, Olander JV, Connolly DT, Stern D. Vascular permeability factor: a tumor-derived polypeptide that induces endothelial cell and monocyte procoagulant activity, and promotes monocyte migration. Journal of Experimental Medicine. 1990:172(6):1535-1545.

84. Ku DD, Zaleski JK, Liu S, Brock T. Vascular endothelial growth factor induces EDRF-dependent relaxation of coronary arteries. American Journal of Physiology. 1993:265:H586-592.

85. Ku DD, Zaleski JK, Liu S, Brock T. Vascular endothelial growth factor induces EDRF-dependent relaxation of coronary arteries. American Journal of Physiology.1993:19265:H586-592.

86. Cuevas P, Garcia-Calvo M, Carceller F, Reimers D, Zazo M, Cuevas B, MunozWillery I, Martinez-Coso V, Lamas S, Gimenez-Gallego G. Correction of hypertension by normalization of endothelial levels of fibroblast growth factor and nitric oxide synthase in spontaneously hypertensive rats. Proceedings of the National Academy of Sciences USA. 1996:93:11996-12001.

87. Robinson, C.J. and S.E. Stringer. The Splice Variants of Vascular Endothelial Growth Factor and Their Receptors. Journal of Cell Science. 2001:114:853.

88. Ferrara N, Houck K, Jakeman L, Leung DW. Molecular and biological 
properties of the vascular endothelial growth factor family of proteins. Endocrine Reviews. 1992:13(1):18-32.

89. Ostendorf T, et al. VEGF165 mediates glomerular endothelial repair. Journal of Clinical Investigation. 1999;104(7):913-923.

90. Byrne, A.M. et al. Angiogenic and cell survival functions of vascular endothelial growth factor (VEGF). Journal of Cellular and Molecular Medicine. 2005:9(4):777-794.

91. Angelo, L.S. and R. Kurzrock. Vascular endothelial growth factor and its relationship to inflammatory mediators. Clinical Cancer Research. 2007:13(10):2825-2830.

92. Houck KA, Leung DW, Rowland AM, Winer J, Ferrara N. Dual regulation of vascular endothelial growth factor bioavailability by genetic and proteolytic mechanisms. Journal of Biological Chemistry. 1992:267:26031-26037.

93. Cohen, T., Gitay-Goren, H., Sharon, R. et al. VEGF121, a vascular endothelial growth factor (VEGF) isoform lacking heparin binding ability, requires cell surface heparan sulfates for efficient binding to the VEGF receptors of human melanoma cells. Journal of Biological Chemistry. 1995:270: 11322-11326.

94. Park JE, Keller G-A, Ferrara N. The vascular endothelial growth factor (VEGF) isoforms: differential deposition into the subepithelial extracellular matrix and bioactivity of ECM-bound VEGF. Molecular Biology of the Cell. 1993:4(12):1317-1326.

95. Keyt B, Berleau L, Nguyen H, Heinshon H, Chen H, Vandlen R, Ferrara N. The carboxyl-terminal domain (111-165) of VEGF is critical for mitogenic potency. Journal of Biological Chemistry. 1996:271(13):7788-7795.

96. Petrova, T. V., Makinen, T., and Alitalo, K. Signaling via vascular endothelial growth factor receptors. Experimental Cell Research. 1999:253(1):117-130.

97. Karkkainen, M.J., Makinen, T. \& Alitalo, K. Lymphatic endothelium: a new frontier of metastasis research. Nature Cell Biology. 2002:4(1): E2-E5.

98. Shalaby, F., Rossant, J., Yamaguchi, T. P., Gertsenstein, M., Wu, X. F., Breitman, M.L., and Schuh, A. C. Failure of blood island formation and vasculogenesis in Flk-1-deficient mice. Nature. 1995:376, 62-66.

99. Fong, G. H., Rossant, J., Gertsenstein, M., and Breitman, M. L. Role of the Flt1receptor tyrosine kinase in regulating the assembly of vascular endothelium. Nature 1995:376(6535):66-70. 
100. Ferrara, N., Gerber H. and LeCouter J. The biology of VEGF and its receptors. Nature Medicine 2003:9:669-676.

101. Shibuya, M. et al. Nucleotide sequence and expression of a novel human receptor-type tyrosine kinase (flt) closely related to the fms family. Oncogene. 1990:5(4): 519-527.

102. Terman, B.I. et al. Identification of a new endothelial cell growth factor receptor tyrosine kinase. Oncogene. 1991:6(9):1677-1683.

103. deVries C, Escobedo JA, Ueno H, Houck KA, Ferrara N, Williams LT. The fms-like tyrosine kinase, a receptor for vascular endothelial growth factor. Science. 1992:255(5047):989-991.

104. Terman BI, Vermazen MD, Carrion ME, Dimitrov D, Armellino DC, Gospodarowicz D, Bohlen P. Identification of the KDR tyrosine kinase as a receptor for vascular endothelial growth factor. Biochemical and Biophysical Research Communications. 1992:187(3):1578-1586.

105. Ziche, M., Jones, J., and Gullino, P.M. Role of prostaglandin E1 and copper in angiogenesis. Journal of the National Cancer Institute. 1982:69(2):475482.

106. Harris, E.D. A requirement for copper in angiogenesis. Nutritional. Reviews. 2004:62(2):60-64.

107. Sen, C.K. et al. Copper-induced vascular endothelial growth factor expression and wound healing. American Journal of Heart and Circulatory Physiology. 2002:282(5): H1821-H1827.

108. Wenger, R.H. Cellular adaptation to hypoxia: O2-sensing protein hydroxylases, hypoxia-inducible transcription factors and O2-regulated gene expression. Federation of American Societies of Experimental Biology Journal. 2002:16(10):1151-1162.

109. Semenza, G.L. Hypoxia-inducible factor 1: master regulator of O2 homeostasis. Current Opinion in Genetic Development. 1998:8(5):588-594.

110. Safran, M. \& Kaeline, W.J. HIF hydroxylation and the mammalian oxygen-sensing pathway. Journal of Clinical Investigation. 2003:111(6), 779783.

111. Jaakkola, P. et al. Targeting of HIF-alpha to the von Hippel-Lindau ubiquitylation complex by O2-regulated prolyl hydroxylation. Science. 2001:292(5516): 468-472. 
112. Ivan, M. et al. HIFalpha targeted for VHL-mediated destruction by proline hydroxylation: implications for $\mathrm{O} 2$ sensing. Science. 2001:292(5516): 464-468.

113. Masson, N. and Ratcliffe, P.J., HIF prolyl and asparaginyl hydroxylases in the biological response to intracellular O2 levels. Journal of Cell Science. 2003:116(Pt 15): 3041-3049.

114. Maxwell, P.H. \& Ratcliffe, P.J. Oxygen sensors and angiogenesis. Seminars in Cell and Developmental Biology. 2002:13(1):29-37.

115. Kallio, P.J. et al. Signal transduction in hypoxic cells: inducible nuclear translocation and recruitment of the $\mathrm{CBP} / \mathrm{p} 300$ coactivator by the hypoxiainducible factor-1alpha. European Molecular Biology Organization Journal. 1998:17(22):6537-6586.

116. Jiang, et. al Dietary copper supplementation reverses hypertrophic cardiomyopathy induced by chronic pressure overload in mice. Journal Experimental Medicine. 2007:204(3):657-666.

117. Takimoto, E. et al. Chronic inhibition of cyclic GMP phosphodiesterase 5A prevents and reverses cardiac hypertrophy. Nature Medicine. 2005:11(2): 214-222.

118. Elsherif, L. Jiang, Y., Saari, J.T., and Kang, Y.J., Dietary copper restriction-induced changes in myocardial gene expression and the effect of copper repletion. Experimental Biology and Medicine. 2004:229(7): 616-622.

119. Shiojima, I. et al. Disruption of coordinated cardiac hypertrophy and angiogenesis contributes to the transition to heart failure. Journal of Clinical Investigation. 2005:115(8): 2108-2118.

120. Izumiya, Y. et al. Vascular endothelial growth factor blockade promotes the transition from compensatory cardiac hypertrophy to failure in response to pressure overload. Hypertension. 2006:47(5): 887-893.

121. Friehs, I. et al. Vascular endothelial growth factor delays onset of failure in pressure-overloaded hypertrophy through matrix metalloproteinase activation and angiogenesis. Basic Research in Cardiology. 2006:101(3):204-213.

122. Lee, L.Y., et al. Focal angiogen therapy using intramyocardial delivery of an adenovirus vector conding for vascular endothelical growth factor 121. Annals of Thoracic Surgery. 2000:69(1):14-23.

123. Friehs, I. et al. Promoting angiogenesis protects severely hypertrophied hearts from ischemic injury. Annals of Thoracic Surgery. 2004:77(6): 2004-2010. 
124. Visconti RP, Richardson CD, Sato TN. Orchestration of angiogenesis and arteriovenous contribution by angiopoietins and vascular endothelial growth factor (VEGF). Proceedings of the National Academy of Sciences USA. 2002: 99(12):8219-24.

125. Senger, D.R. et al. Tumor cells secrete a vascular permeability factor that promotes accumulation of ascites fluid. Science 1983:219(4587): 983-985.

126. Dvorak, H.F. et al. Vascular permeability factor/vascular endothelial growth factor, microvascular hypermeability, and angiogenesis. American Journal of Pathology. 1995:146(5): 1029-1039.

127. Tham E, Wang J, Piehl F, Weber G. Upregulation of VEGF-A without angiogenesis in a mouse model of dilated cardiomyopathy caused by mitochondrial dysfunction. Journal of Histochemistry and Cytochemistry. 2002: 50(7): 935-44.

128. Tsai, EJ and Kass DA. Cyclic GMP signaling in cardiovascular pathophysiology and therapeutics. Pharmacology and Therapeutics. 2009:122(3): 216-238.

129. Hofmann, F. The biology of cyclic GMP-dependent protein kinases. Journal of Biological Chemistry. 2005:280(1):1-4.

130. Komalavilas, P. et al. Activation of mitogen-activated protein kinase pathways by cyclic GMP and cyclic GMP-dependent protein kinase in contractile vascular smooth muscle cells. Journal of Biological Chemistry. 1999:274(48):34301-34309.

131. Lincoln, TM and Cornwell TL. Intracellular cyclic GMP receptor proteins. Federation of American Societies of Experimental Biology Journal. 1993:7(2):328-338.

132. Feil R. et al. Cyclic GMP-dependent protein kinases and the cardiovascular system: insights from genetically modified mice. Circulation Research. 2003:93(10):907-916.

133. Lukowski, R. Cardiac hypertrophy is not amplified by deletion of cGMPdependent protein kinase I in cardiomyocytes. Proceedins of the National Academy of SciencesU.S.A. 2010:107(12): 5646-5651.

134. Ammendola, A. et al. Molecular determinants of the interaction between the inositol 1,4,5-trisphosphate receptor-associated cGMP kinase substrate (IRAG) and cGMP kinase Ibeta. Journal of Biological Chemistry. 2001:276(26): 24153-24159. 
135. Surks, H.K. et al. Regulation of myosin phosphatase by a specific interaction with cGMP- dependent protein kinase Ialpha.

Science.1999:286(5444):1583-1587.

136. Burgoyne, J.R. et al. Cysteine redox sensor in PKGIa enables oxidantinduced activation. Science. 2007:317(5843):1393-1397.

137. Murry CE, Jennings RB, Reimer KA. Preconditioning with ischemia: a delay of lethal cell injury in ischemic myocardium. Circulation. 1986:74: 11241136.

138. Kishimoto I et al. A genetic model provides evidence that the receptor for atrial natriuretic peptide (guanylyl cyclase-A) inhibits cardiac ventricular myocyte hypertrophy. Proceedings of the National Academy of Sciences USA. 2001:98: 2703-2706.

139. Zahabi A. et al. Expression of constitutively active guanylate cyclase in cardiomyocytes inhibits the hypertrophic effects of isoproterenol and aortic constriction on mouse hearts. Journal of Biological Chemistry. 2003:278(48): 27694-47699.

140. Wollert KC et al. Gene transfer of cGMP-dependent protein kinase I enhances the antihypertrophic effects of nitric oxide in cardiomoyocytes. Hypertension 2002:39(1): 87-92.

141. Knowles JW et al. Pressure-independent enhancement of cardiac hypertrophy in natriuretic peptide receptor A-deficient mice. Journal of Clinical Investigation. 2001:107(8): 975-984.

142. Koneru $\mathrm{S}$ et al. Sildenafil-mediated neovascularization and protection against myocardial ischaemia reperfusion injury in rats: role of VEGF/angiopoietin-1. Journal of Cellular and Molecular Medicine. 2008:12(6B) :2651-64.

143. Senthilkumar, A et al. Sildenafil promotes ischemia-induced angiogenesis through a PKG-dependent pathway. Arteriosclerosis Thrombosis Vascular Biology. 2007: 27(9):1947-195.

144. Lukowski $\mathrm{R}$ et al. Cardiac hypertrophy is not amplified by deletion of cGMP-dependent protein kinase I in cardiomyocytes. Proceedings of the National Academy of Sceinces USA. 2010:107(12):5646-5651.

145. Forfia, PR et al. Acute phosphodiesterase 5 inhibition mimics hemodynamic effects of B-type natriuretic peptide and potentiates B-type 
natriuretic peptide effects in failing but not normal canine heart. Journal of the American College of Cardiology. 2007:49(10):1079-1088.

146. Corbin JD et al. High lung PDE5: a strong basis for treating pulmonary hypertension with PDE5 inhibitors. Biochemical and Biophysical Research Communications. 2005:334(3): 930-938.

147. Nagendran, J. et al. Phosphodiesterase type 5 is highly expressed in the hypertrophied human right ventricle, and acute inhibition of phosphodiesterase type 5 improves contractility. Circulation 2007:116(3): 238248.

148. Kukreja RC et al. Pharmacological preconditioning with sildenafil: basic mechanisms and clinical implications. Vascular Pharmacology. 2005:42(5-6): 219-232.

149. Das A., Xi, L. \& Kukreja, R.C. Phosphodiesterase-5 inhibitor sildenafil preconditions adult cardiac myocytes against necrosis and apoptosis. Essential role of nitric oxide signaling. Journal of Biological Chemistry. 2005:280(13): 12944-12955.

150. Das A. et al. Protein kinase C plays an essential role in sildenafil-induced cardioprotection in rabbits. American Journal of Physiology. 2004:286(4): H1455-H1460.

151. Ockaili $\mathrm{R}$ et al. Sildenafil (Viagra) induces powerful cardioprotective effect via opening of mitochondrial K(ATP) channels in rabbits. American Journal of Physiology. 283(3):H1263-H1269.

152. Salloum F et al. Sildenafil induces delayed preconditioning through inducible nitric oxide synthase-dependent pathway in mouse heart. Circulation Research. 2003:92(6):595-597.

153. Das A et al. Phosphodiesterase-5 inhibitor sildenafil preconditions adult cardiac myocytes against necrosis and apoptosis. Essential role of nitric oxide signaling. Journal of Biological Chemistry. 2005:280(13):12944-12955.

154. Salloum, FN et al. Sildenafil (Viagra) attenuates ischemic cardiomyopathy and improves left ventricular function in mice. American Journal of Physiology. 2008:294(3): H1398-H1406.

155. Fisher PW et al. Phosphodiesterase-5 inhibition with sildenafil attenuates cardiomyocyte apoptosis and left ventricular dysfunction in a chronic model of doxorubicin cardiotoxicity. Circulation. 2005:111(13):1601-1610. 
156. Salloum, FN. Et al. Vardenafil: a novel type 5 phosphodiesterase inhibitor reduces myocardial infarct size following ischemia/reperfusion injury via opening of mitochondrial K(APT) channels in rabbits. Journal of Molecular andCellular Cardiology. 2006:40(3): 405-411.

157. Sesti $\mathrm{C}$ et al. The phosphodiesterase-5 inhibitor tadalafil reduces myocardial infarct size. International Journal of Impotence Research. 2007:19(1):55-61.

158. Hardt, SE \& Sadoshima J. Glycogen synthase kinase-3 3 : a novel regulator of cardiac hypertrophy and development. Circulation Research. 2002:90(10):1055-1063.

159. Kaga S. et al. Glycogen synthase kinase-3beta/beta-catenin promotes angiogenic and anti-apoptotic signaling through the induction of VEGF, Bcl-2 and survivin expression in rat ischemic preconditioned myocardium. Journal of Molecular and Cellular Cardiology. 2006:40(1):138-147.

160. Das, A. et al. Protein kinase G-dependent cardioprotective mechanism of phosphodiesterase-5 inhibition involves phosphorylation of ERK and GSK3beta. Journal of Biological Chemistry. 2008:283(43):29572-29585.

161. Nishi J et al. Vascular endothelial growth factor receptor-1 regulates postnatal angiogenesis through inhibition of the excessive activation of Akt. Circulation Research. 2008:103(3):261-268.

162. Fujio $\mathrm{Y}$ et al. Akt promotes survival of cardiomyocytes in vitro and protects against ischemia-reperfusion injury in mouse heart. Circulation. 2000:101(6):660-667.

163. Matsui T. et al. Akt activation preserves cardiac function and prevents injury after transient cardiac ischemia in vivo. Circulation 2001:104(3): 330-335.

164. Ku N-O, Zhou X, Toivola DM, Omary MB. The cytoskeleton of digestive epithelia in health and disease. American Journal of Physiology. 1999;277(6 Pt 1):G1108-G1137.

165. Fuchs E, Cleveland DW. A structural scaffolding of intermediate filaments in health and disease. Science. 1998:279(5350):514-519.

166. Cleveland D and Fuchs E. A Structural Scaffolding of Intermediate Filaments in Health and Disease. Science. 1998:279(5350):514-519.

167. E. Fuchs and K. Weber. Intermediate filaments: structure, dynamics, function, and disease. Annual Review of Biochemistry. 1994:63:345-82. 
168. Colucci-Guyon E et al. Mice Lacking Vimentin Develop and Reproduce without an Obvious Phenotype. Cell. 1994:79(4): 679-694.

169. Ando J et al. Changes in cell morphology and cytoskeletal organization are induced by human mitotic checkpoint gene, Bubl. Biochemical and Biophysical Research Communications 2008:365(4):691-69.

170. Eckes, B. et al. Impaired wound healing in embryonic and adult mice lacking vimentin. Journal of Cell Science. 2000:113(Pt13): 2455-2462.

171. Eckes, B. et al. Impaired mechanical stability, migration and contractile capacity in vimentin-deficient fibroblasts. Journal of Cell Science. 1998: 111(Pt13): $1897-1907$.

172. Schiffers PMH et al. Altered Flow-Induced Arterial Remodeling in Vimentin-Deficient Mice. Arteriosclerosis Thrombosis and Vascular Biology. 2000:20(3):611-616.

173. Siddiqui, et al. Inhibition of phenylephrine-induced cardiac hypertrophy by docosahexaenoic acid. Journal of Biological Chemistry. 2004:92(6), 11411159.

174. Chen et al. Hydrogen peroxide dose dependent induction of cell death or hypertrophy in cardiac myocytes. Archives of Biochemistry and Biophysics. 2000:373(1): 242-248.

175. Madonna R and Caterina R. VEGF receptor switching in heart development and disease. Cardiovascular Research. 2009:84(1):4-6.

176. Takimoto E, Champion HC, Li M, Belardi D, Ren S, Rodriguez ER et al. Chronic inhibition of cyclic GMP phosphodiesterase 5A prevents and reverses cardiac hypertrophy. Nature Medicine. 2005:11(2):214-222.

177. Zhou Y, Jiang Y Kang YJ. Copper reverses cardiomyocyte hypertrophy through vascular endothelial growth factor-mediated reduction in the cell size. Journal of Molecuar and Cellular Cardiology. 2008: 45(1):106-117.

178. Seetharam L et al. A unique signal transduction from FLT tyrosine kinase, a receptor for vascular endothelial growth factor VEGF. Oncogene 1995:10(1):135-147.

179. Waltenberger J et al. Different signal transduction properties of KDR and Flt1, two receptors for vascular endothelial growth factor. Journal of Biological Chemistry. 1994;269(43):26988-26995. 
180. Reynolds AR et al. Elevated Flk1 (vascular endothelial growth factor receptor 2) signaling mediates enhanced angiogenesis in beta3-integrin-deficient mice. Cancer Research. 2004:64(23):8643-8650.

181. Shiojima I et al. Disruption of coordinated cardiac hypertrophy and angiogenesis contributes to the transition to heart failure. Journal of Clinical Investigation. 2005: 115(8):2108- 2118.

182. Izumiya $\mathrm{Y}$ et al. Vascular endothelial growth factor blockade promotes the transition from compensatory cardiac hypertrophy to failure in response to pressure overload. Hypertenstion. 2006:47(5):887-893.

183. Clark DE et al. A vascular endothelial growth factor antagonist is produced by the human placenta and released into the maternal circulation. Biology of Reproduction. 1998:59(6):1540-1548.

184. Zeng H, Dvorak HF, Mukhopadhyay D. Vascular permeability factor (VPF)/vascular endothelial growth factor (VEGF) receptor-1 down-modulates VPF/VEGF receptor- 2 mediated endothelial cell proliferation, but not migration, through phosphotidylinositol 3-kinase-dependent pathways. Journal of Biological Chemistry. 2001:276(29):26969-26979.

185. Rahimi N, Dayanir V, Lashkari K. Receptor chimeras indicate that the vascular endothelial growth factor receptor-1 (VEGFR1) modulates mitogenic activity of VEGFR2 in endothelial cells. Journal of Biological Chemistry. 2000;275(22):16986-16992.

186. Autiero M et al. Role of PIGF in the intra- and intermolecular cross talk between VEGF receptors Flt1 and FLK1. Nature Medicine. 2003:9(7):936-943.

187. Takimoto Et al. Chronic inhibition of cyclic GMP phosphodiesterase 5A prevents and reverses cardiac hypertrophy. Nature Medicine. 2005:11(2):214-222.

188. Eckes, B. et al. Impaired mechanical stability, migration and contractile capacity in vimentin-deficient fibroblasts. Journal of Cell Science. 1998:111(Pt13): 1897-1907.

189. Traub, P. Intermediate Filaments: A Review (Springer-Verlag, New York, 1985).

190. Christian JL, Edelstein NG \& Moon RT. Overexpression of wild-type and dominant negative mutant vimentin subunits in developing Xenopus embryos. New Biologist. 1990:2(8):700-711. 
191. Eckes B et al. Impaired mechanical stability, migration and contractile capacity in vimentin-deficient fibroblasts. Journal Cell Science. 1998:111(Pt13), 1897-1907.

192. Eckes B et al. Impaired wound healing in embryonic and adult mice lacking vimentin. Journal Cell Science. 2000:113(Pt13): 2455-2462.

193. Mor-Vaknin, $\mathbf{N}$ et al. Vimentin is secreted by activated macrophages. Nature Cell Biology. 2002:5(1):59 - 63.

194. Turowski P et al. Vimentin dephosphorylation by protein phosphatase 2A is modulated by the targeting subunit B55. Molecular Biology of the Cell. 1999:10(6):1997-2015.

195. Yasui $Y$ et al. Protein kinases required for segregation of vimentin filaments in mitotic process. Oncogene. 2001:20(23):2868-2876.

196. Takeisi Y et al. Transgenic overexpression of Constitutively Active Protein Kinase C e Causes Concentric Cardiac Hypertrophy. Circulation Research. 2000:86(12): 1218-1223.

197. Palaniyandi S et al. Protein kinase $C$ in heart failure: a therapeutic target? Cardiovascular Research. 2009:82(2):229-239.

198. Hanh H et al. Protein Kinase C Negatively Regulates Systolic and Diastolic Function in Pathological Hypertrophy. Circulation Research. 2003:93(11):1111-1119.

199. Braz JC et al. PKC alpha regulates the hypertrophic growth of cardiomyocytes through extracellular signal-regulated kinase 1/2 (ERK1/2). Journal of Cell Biology. 2002:156(5):905-19.

200. De Witt Hammer. Small molecule kinase inhibitors in glioblastoma: a systematic review of clinical studies. Neurological Oncology. 2010:12(3):304-16.

201. Rades, D, Setter C, Dunst J, Dahl O, Schild SE, Noack F. Prognostic impact of VEGF and VEGF Receptor 1 (FLT1) expression in patients irradiated for stage IV/III non-small cell lung cancer (NSCLC). Strahlenther Onkologie. 2010:186(6):307-14. 
APPENDIX

\section{List of Abbreviations}

ANP

ANOVA

BrdU

$\mathrm{CaCl}_{2}$

$\mathrm{CCO}$

cGMP

$\mathrm{Cu}$

$\mathrm{CuSO}_{4}$

$\mathrm{CO}_{2}$

$\mathrm{ddH}_{2} \mathrm{O}$

FACS

FBS

Flk

Flt

FSC

HBSS

HEPES

$\mathrm{H}_{2} \mathrm{O}_{2}$
Atrial Natriuretic Peptide Analysis of Variance 5'-Bromo-2'-DeoxyUridine Calcium Chloride

Cytochrome C Oxidase Guanosine 3',5'-cyclic monophosphate Copper Copper Sulfate Carbon Dioxide Double Distilled Water Fluorescence-Activated Cell Sorting Fetal Bovine Serum Fetal Liver Kinase 1 fms-Like Tyrosine Kinase 1 Forward Scatter Light Hanks' Balanced Salt Solution 4-(2-HydroxyEthyl)-1-PiperazineEthaneSulfonic Acid Hydrogen Peroxide 
KDR

Kinase insert Domain-containing Receptor

$\mathrm{MgCl}_{2}$

Magnesium Chloride

$\mathrm{NaCl}$

Sodium Chloride

$\mathrm{NaHCO}_{3}$

Sodium Bicarbonate

$\mathrm{NaOH}$

Sodium Hydroxide

PBS

Phosphate Buffered Saline

PDE

Phosphodiesterase

PE

Phenylephrine

PKC

Protein Kinase C

PKG

Protein Kinase G

PMSF

Phenylmethanesulfonyl Fluoride

Rp-8-CPT-cGMPS

8-(4-Chlorophenylthio)-guanosine 3',5'-cyclic monophosphorothioate, $\mathrm{Rp}$ Isomer triethylammonium salt

RTK

SD

SOD

SSC

siRNA

TNF- $\alpha$

VEGF

VEGFR
Tryosine Kinase Receptor

Standard Deviation

Superoxide Dismutase

Side Scatter Light

Small Interfering RNA

Tumor Nectrosis Factor- $\alpha$

Vascular Endothelial Growth Factor

VEGF Receptor 


\section{CURRICULUM VITAE}

\section{Katherine S. Bourcy}

505 S. Hancock Street

Louisville, KY 40202

Phone: 502.852 .6284

katie.bourcy@louisville.edu

\section{Education}

Ph.D. Pharmacology \& Toxicology, University of Louisville, August 2011

M.S. Pharmacology \& Toxicology, University of Louisville, August 2008

B.S. Biology, University of Louisville, 2002

\section{Graduate Research}

Research Assistant

Y. James Kang

Research Assistant in Cardiovascular Toxicology

Research Assistant

Department of Pharmacology \& Toxicology
2007- July 2011

University of Louisville

2006-2007

University of Louisville

\section{Undergraduate \& Post-baccalaureate Research}

Laboratory Technician

2003-2004

Frank Adamsky, Ph.D.

Agentase, LLC Pittsburgh, PA

Tested and analyzed enzymatic-polymer for pesticide remediation.

Research Assistant

2001-2002

David Schultz, Ph.D.

University of Louisville

Studied the production of unusual monoenoic fatty acids in transgenic plants.

\section{Publications, Theses \& Posters}

Bourcy K, Feng W, Kang YJ. The Involvement of Vimentin in Copper

Regression of Hypertrophy in Human Cardiac Myocytes. In Process.

Zhan L, Hussain LR, Yao Y, Feng W, Bourcy K, Eaton J, Johnson WT, Moraes CT, Kang YJ. Recovery of cytochrome c oxidase activity is required for dietary copper supplementation-induced regression of cardiac hypertrophy in mice. In process.

Bourcy K, Zhou Y, Feng W, Kang YJ. Association of Vascular Endothelial Growth Factor Receptor 1 with cGMP-dependent Protein Kinase-1 is involved in Copper-induced Regression of Cardiac Myocyte Hypertrophy in Cultures. Poster Presentation, March 2010, Society of Toxicology, Salt Lake City, Utah. 
Zhou Y, Bourcy K, Kang YJ. Copper-induced regression of cardiomyocyte hypertrophy is associated with enhanced vascular endothelial growth factor receptor-1 signaling pathway. Cardiovasc Res. 2009 Oct 1;84(1):54-63.

\section{Graduate Honors and Awards}

NIH Pre-doctoral Trainee (T32-ES011564), 2009- 2011

University of Louisville School of Medicine Fellowship, 2006

\section{Undergraduate Honors and Awards}

PLEN Alumni (Fall 2004)

Received over $\$ 10,000$ in merit-based scholarships

\section{Activities}

Foreign languages (Spanish, French \& Brazilian Portuguese)

Outdoor activities (Running, Biking, Hiking)

No Kill Louisville volunteer (Grant Writing Committee, Events \& Publicity committee)

Bully Brigade of No Kill Louisville, Founder \& Chair 